\title{
MHD Turbulence, Turbulent Dynamo and Applications
}

\author{
Andrey Beresnyak and Alex Lazarian
}

\section{Introduction}

Historically, most of the turbulence studies were concerned with non-conductive fluids, described by the Navier-Stokes equations. This is because most fluids present on Earth are non-conductive. In the context of a larger Cosmos, this situation is not a rule but rather an exception. Indeed, space is filled with ionizing radiation and only the protection of our atmosphere, which is very dense by astronomical standards, allows us to have a big volumes of insulating fluids, such as the atmosphere and the oceans. In contrast, most of the ordinary matter in the Universe is ionized, i.e. in a state of plasma. The description of ionized, well-conductive fluids must include the Lorentz force and the induction equation for the magnetic field. As it turned out, turbulent conductive fluids tend to quickly generate their own magnetic fields in the process known as dynamo. On the other hand, the presence of the dynamically important magnetic field could be considered an observational fact. In spiral galaxies magnetic field has a regular component, usually along the arms and a random turbulent component of the same order. The value of the magnetic field, around $5 \mu G$, roughly suggests equipartition between magnetic and kinetic forces.

Observations of magnetized turbulence in the interstellar medium, galaxy clusters and the solar wind have confirmed that turbulence is indeed ubiquitous in astrophysical flows and has been detected in almost all astrophysical and space environments, see, e.g., [1, 2, 3]. The Reynolds numbers of astrophysical turbulence are, typically, very high, owing to astrophysical scales which are enormous compared to dissipative scales. Recent years have been marked by new understanding of the key role that turbulence plays in a number of astrophysical processes [4, 5]. Most

$\begin{aligned} & \text { Andrey Beresnyak } \\ & \text { Ruhr-Universität } \quad \text { Bochum, }\end{aligned}$
$\begin{array}{ll}\text { andrey.at.astro@gmail.com }\end{array} \quad 44780 \quad$ Bochum, Germany, e-mail:

A. Lazarian

University of Wisconsin-Madison, WI, USA e-mail: lazarianeastro.wisc.edu 
notably, turbulence has drastically changed the paradigms of interstellar medium and molecular cloud evolution [6, 7, 8], see also review [9]. While small scale, kinetic turbulence has been probed by a variety of approaches such as gyrokinetics, Hall MHD and electron MHD [10, 11, 12], in this review we concentrate mostly on the fluid-scale MHD turbulence which is the most important for star formation and interaction with cosmic rays.

The theoretical understanding of magnetized turbulence can be roughly subdivided in two big domains: MHD dynamo and the inertial range of MHD turbulence. In the first part of this review we will explain small-scale dynamo, which is fast, universal mechanism to wind up magnetic fields. The second half is devoted to the properties of the inertial range cascades of incompressible and compressible MHD turbulence. In particular we discuss both the spectrum of fundamental MHD modes and the intermittency properties of turbulence. The third part is devoted to applications of our knowledge of MHD cascades, which includes turbulent reconnection, cosmic ray propagation [13] and damping of instabilities [14].

\section{Astrophysical Dynamo}

One of the central processes of MHD dynamics is how conductive fluid generates its own magnetic field, a process known broadly as "dynamo". Turbulent dynamo has been subdivided into "large-scale/mean-field dynamo" and "small-scale/fluctuation dynamo" depending on whether magnetic fields are amplified on scales larger or smaller than outer scale of turbulence.

Although several "no-dynamo" theorems have been proved for flows with symmetries, a generic turbulent flow, which possesses no exact symmetry, was expected to amplify magnetic field by stretching, due to the particle separation in a turbulent flow. For the large-scale dynamo, a "twist-stretch-fold" mechanism was introduced [15]. Turbulent flow possessing perfect statistical isotropy can not generate large-scale field, so the observed large-scale fields, such as in the disk galaxies, are generated when statistical symmetries of turbulence are broken by large-scale asymmetries of the system, such as stratification, rotation and shear, see, e.g., [16, 17]. Large-scale dynamo is often investigated using so-called mean field theory, see, e.g. [18], where the magnetic and velocity field are decomposed into mean and fluctuating parts and the equations for the mean field are closed using statistical or volume averaging over the fluctuating turbulent part.

The studies of large-scale dynamo are very rich and diverse due to the variety of conditions in astrophysical flows in different objects, one of the most ambitious goals is to explain the solar cycle, see, e.g., [19]. In this review we decided to concentrate on the small-scale dynamo as it is fast and generic and almost always generate magnetic fluctuations with energy of the order of the kinetic energy (so-called equipartition). Magnetic fluctuations could be subsequently ordered by slower largescale dynamo and produce large-scale magnetic fields. Some objects, such as galaxy clusters, are dominated by small-scale dynamo, however. 


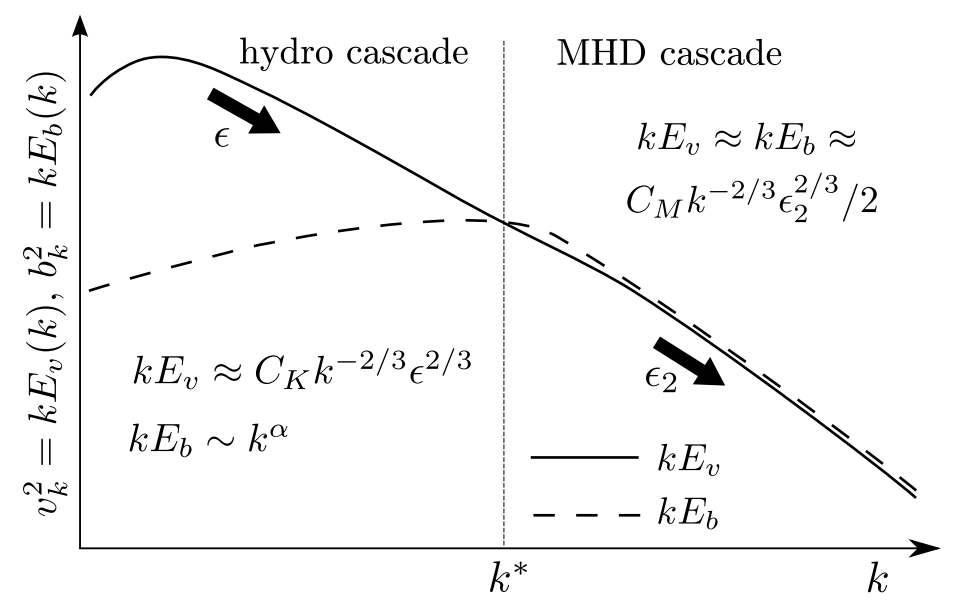

Fig. 1 A cartoon of kinetic and magnetic spectra in small-scale dynamo, at a particular moment of time when equipartition wavenumber is $k^{*}$.

Most studied was so-called kinematic regime of small-scale dynamo, which ignores the backreaction of the magnetic field [20, 21, 22]. However, from these models it was not clear whether magnetic energy will continue to grow after the end of kinematic regime. In astrophysical objects with very large Re it becomes inapplicable at very short timescales. Also magnetic spectrum of kinematic dynamo, possessing positive spectral index, typically $3 / 2$, is incompatible with observations in galaxy clusters [23]. These observations clearly indicate steep spectrum with negative power index at small scales. In fact, from theoretical viewpoint, kinematic dynamo is inapplicable in most astrophysical environment, because the Alfvén speed is typically many orders of magnitude higher than the Kolmogorov velocity.

The understanding of nonlinear small-scale dynamo was developing slowly and was influenced by analytical kinematic studies. For a long time a popular belief was that after becoming nonlinear the small-scale dynamo will saturate in one way or another. If we assume that the magnetic energy indeed saturates as soon as the dynamo become nonlinear. The saturation level in this case will be $\rho v_{\eta}^{2} / 2$, where $v_{\eta}$ is a Kolmogorov velocity scale. This is a factor of $R e^{-1 / 2}$ smaller than the kinetic energy density and is completely unimportant in high-Re astrophysical environments. The price tag to discover what will happen in the nonlinear regime was, therefore, fairly high. The early work by Schlüter and Bierman [24] suggested that the dynamo will continue to grow and will saturate on each subsequent scale by its dynamical time. The true revival of small-scale dynamo happened relatively recently due to availability of direct numerical simulations. First simulations were concerned with saturated state of small scale dynamo and produced steep spectrum at small scales and significant outer-scale fields, and the saturated state seems to be only weakly dependent on $R e$ and $P r$ as long as $R e$ is large, see, e.g, [25]. Furthermore it was suggested in [11, 26, 27, 28, 29] that there is a linear growth stage. In subsequent 
sections we will follow the argumentation of [29], who provided sufficient analytical and numerical argumentation behind the universality of the nonlinear small-scale dynamo.

\subsection{Universal Nonlinear Small-Scale Dynamo}

We assume that the spectra of magnetic and kinetic energies at a particular moment of time are similar to what is presented on Fig. 11. Magnetic and kinetic spectra cross at some "equipartition" scale $1 / k^{*}$, below which both spectra are steep due to MHD cascade [30, 31]. This assumption is suggested by both numerical evidence [32, 26] and observations of magnetic fields in clusters of galaxies [23]. Also, if we start with assuming $\operatorname{Pr}=1$ and magnetic energy very small and follow standard kinematic dynamo calculations, e.g. [22] the magnetic energy will grow exponentially till the magnetic spectrum intersect kinetic spectrum at the viscous scales. This will roughly correspond to the beginning of the nonlinear regime with equipartition scale equal to the dissipation scale.

At larger scales magnetic spectrum is shallow, $k^{\alpha}, \alpha>0$, while kinetic spectrum is steep due to the hydro cascade. Most of the magnetic energy is concentrated at scale $1 / k^{*}$. We designate $C_{K}$ and $C_{M}$ as Kolmogorov constants of hydro and MHD respectively. The hydrodynamic cascade rate is $\varepsilon$ and the MHD cascade rate as $\varepsilon_{2}$. Due to the conservation of energy in the inertial range, magnetic energy will grow at a rate $\varepsilon-\varepsilon_{2}$. We will designate $C_{E}=\left(\varepsilon-\varepsilon_{2}\right) / \varepsilon$ as an "efficiency of the small-scale dynamo" and will argue that this is a true constant, since: a) turbulent dynamics is local in scale in the inertial range; b) neither ideal MHD nor Euler equations contain any scale explicitly. Magnetic energy, therefore, grows linearly with time if $\varepsilon=$ const. The equipartition scale $1 / k^{*}$ will grow with time as $t^{3 / 2}[28]$. This is equivalent to saying that small-scale dynamo saturates at several dynamical times at scale $1 / k^{*}$ and proceeds to a twice larger scale [11]. If magnetic energy grows approximately till equipartition [25, 26], the whole process will take around several dynamical timescales of the system, or more quantitatively, $\left(C_{K}^{3 / 2} / C_{E}\right)\left(L / v_{L}\right)$.

\subsection{Locality of the Small-Scale Dynamo}

We will use "smooth filtering" approach with dyadic-wide filter in k-space [33]. We designate a filtered vector quantity as $\mathbf{a}^{[k]}$ where $k$ is a center of a dyadic Fourier filter in the range of wave numbers $[k / 2,2 k]$. The actual logarithmic width of this filter is irrelevant to further argumentation, as long as it is not very small. We will assume that the vector field $\mathbf{a}$ is Hölder-continuous, i.e., $|\mathbf{a}(\mathbf{x})-\mathbf{a}(\mathbf{y})|<|\mathbf{x}-\mathbf{y}|^{\mathbf{h}}$ with exponent $0<h<1$ and designate $a_{k}=\left\langle\left|\mathbf{a}^{[k]}\right|^{3}\right\rangle^{1 / 3}$ (angle brackets are averages over ensemble), which is expected to scale as $a_{k} \sim k^{\sigma_{3}}$, e.g., $k^{-1 / 3}$ for ve-

locity in Kolmogorov turbulence. The energy cascade rate is $\varepsilon=C_{K}^{-3 / 2} k v_{k}^{3}$, where 
Table 1 Transfers and Upper Limits

\begin{tabular}{rllcc}
\hline \hline Transfers & & & $p \ll k$ & $q \gg k$ \\
\hline$T_{v v}(p, k)$ & $=$ & $-\left\langle\mathbf{v}^{[k]}(\mathbf{v} \cdot \nabla) \mathbf{v}^{[p]}\right\rangle$ & $p v_{p} v_{k}^{2}$ & $k v_{k} v_{q}^{2}$ \\
$T_{b b}(p, k)$ & $=$ & $-\left\langle\mathbf{b}^{[k]}(\mathbf{v} \cdot \nabla) \mathbf{b}^{[p]}\right\rangle$ & $p b_{p} v_{k} b_{k}$ & $k b_{k} v_{q} b_{q}$ \\
$T_{v b}(p, k)$ & $=$ & $\left\langle\mathbf{b}^{[k]}(\mathbf{b} \cdot \nabla) \mathbf{v}^{[p]}\right\rangle$ & $p v_{p} b_{k}^{2}$ & $k b_{k} v_{q} b_{q}$ \\
$T_{b v}(p, k)$ & $=$ & $\left\langle\mathbf{v}^{[k]}(\mathbf{b} \cdot \nabla) \mathbf{b}^{[p]}\right\rangle$ & $p b_{p} v_{k} b_{k}$ & $k v_{k} b_{q}^{2}$ \\
$T_{w^{+} w^{+}}(p, k)$ & $=$ & $-\left\langle\mathbf{w}^{+[k]}\left(\mathbf{w}^{-} \cdot \nabla\right) \mathbf{w}^{+[p]}\right\rangle$ & $p w_{p} w_{k}^{2}$ & $k w_{k} w_{q}^{2}$ \\
\hline
\end{tabular}

we defined Kolmogorov constant $C_{K}$ by third order, rather than second order quantities. We will keep this designation, assuming that traditional Kolmogorov constant could be used instead. We use spectral shell energy transfer functions such as $T_{v v}(p, k)=-\left\langle\mathbf{v}^{[k]}(\mathbf{v} \cdot \nabla) \mathbf{v}^{[p]}\right\rangle, T_{w^{+}{ }^{+}}(p, k)=-\left\langle\mathbf{w}^{+[k]}\left(\mathbf{w}^{-} \cdot \nabla\right) \mathbf{w}^{+[p]}\right\rangle$ [34], applicable to incompressible ideal MHD equations, where $w^{ \pm}$are Elsässer variables and $v, b$ and $w^{ \pm}$are measured in the same Alfvénic units. Using central frequency $k$ and studying "infrared" (IR) transfers from $p \ll k$, and "ultraviolet" (UV) transfers, from $q \gg k$, we will provide absolute bounds on $|T|$, in units of energy transfer rate as in [33, 35], and relative volume-averaged bounds which are divided by the actual energy rate and are dimensionless. We will consider three main $k$ intervals presented on Fig. 1, $k \ll k^{*}$ ("hydro cascade"), $k \sim k^{*}$ ("dynamo") and $k \gg k^{*}$ ("MHD cascade").

\subsection{MHD cascade, $k \gg k^{*}$}

The only energy cascades here are Elsässer cascades and, by the design of our problem, $w^{+}$and $w^{-}$have the same statistics, so we will drop \pm . For an exchange with $p \ll k$ band, for $\left|T_{w w}\right|$, using Hölder inequality and wavenumber conservation we get an upper bound of $p w_{p} w_{k}^{2}$ and for $q \gg k$ band it is $k w_{q}^{2} w_{k}$, these bounds are asymptotically small. For the full list of transfers and limits refer to Table 1 . The relative bound should be taken with respect to $C_{M}^{-3 / 2} k w_{k}^{3}$, where $C_{M}$ is a Kolmogorov constant for MHD, from which we get that most of the energy transfer with the $[k]$ band should come from $\left[k C_{M}^{-9 / 4}, k C_{M}^{9 / 4}\right]$ band, see [31]. The global transfers between kinetic and magnetic energy must average out in this regime, nevertheless, the pointwise IR and UV transfers can be bounded by $p b_{p} v_{k} b_{k}$ and $k b_{q}^{2} v_{k}$ and are small [35].

\subsection{Hydro cascade, $k \ll k^{*}$}

Despite having some magnetic energy at these scales, most of the energy transfer is dominated by velocity field. Indeed, $\left|T_{v v}\right|$ is bounded by $p v_{p} v_{k}^{2}$ for $p \ll k$ and 
Table 2 Three-dimensional MHD Dynamo Simulations

\begin{tabular}{ccccccc}
\hline \hline Run & $\mathrm{n}$ & $N^{3}$ & Dissipation & $\langle\varepsilon\rangle$ & $\operatorname{Re}$ & $C_{E}$ \\
\hline M1-6 & 6 & $256^{3}$ & $-7.6 \cdot 10^{-4} k^{2}$ & 0.091 & 1000 & $0.031 \pm 0.002$ \\
M7-9 & 3 & $512^{3}$ & $-3.0 \cdot 10^{-4} k^{2}$ & 0.091 & 2600 & $0.034 \pm 0.004$ \\
M10-12 & 3 & $1024^{3}$ & $-1.2 \cdot 10^{-4} k^{2}$ & 0.091 & 6600 & $0.041 \pm 0.005$ \\
M13 & 1 & $1024^{3}$ & $-1.6 \cdot 10^{-9} k^{4}$ & 0.182 & - & $0.05 \pm 0.005$ \\
M14 & 1 & $1536^{3}$ & $-1.5 \cdot 10^{-15} k^{6}$ & 0.24 & - & $0.05 \pm 0.005$ \\
\hline
\end{tabular}

by $k v_{q}^{2} v_{k}$ for $q \gg k$. Compared to these, $\left|T_{b v}\right|$ transfers are negligible: $p b_{p} v_{k} b_{k}$ and $k b_{q}^{2} v_{k}$. For magnetic energy in $p \ll k$ case we have $\left|T_{v b}\right|$ and $\left|T_{b b}\right|$ transfers bounded by $p v_{p} b_{k}^{2}, p b_{p} v_{k} b_{k}$ and for $q \gg k$ case $\left|T_{v b}\right|$ and $\left|T_{b b}\right|$ are bounded by $k b_{k} v_{q} b_{q}$. Out of these three expressions the first two go to zero, while the third goes to zero if $\alpha-$ $2 / 3<0$ or have a maximum at $q=k^{*}$ if $\alpha-2 / 3>0$. This means that for the transfer to magnetic energy we have IR locality, but not necessarily UV locality. Note that magnetic energy for $k \ll k^{*}$ is small compared to the total, which is dominated by $k=k^{*}$. We will assume that $\alpha-2 / 3>0$ and that the spectrum of $b_{k}$ for $k<k^{*}$ is formed by nonlocal $\left|T_{v b}\right|$ and $\left|T_{b b}\right|$ transfers from $k^{*}$, namely magnetic structures at $k$ are formed by stretching of magnetic field at $k^{*}$ by velocity field at $k$. Magnetic spectrum before $k^{*}$ is, therefore, nonlocal and might not be a power-law, but our further argumentation will only require that $b_{k}<v_{k}$ for $k<k^{*}$.

\subsection{Dynamo cascade $k=k^{*}$}

In this transitional regime our estimates of Elsässer UV transfer and kinetic IR transfer from two previous sections will hold. We are interested how these two are coupled together and produce observed magnetic energy growth. IR $p \ll k^{*}\left|T_{v b}\right|$ and $\left|T_{b b}\right|$ transfers will be bounded by $p v_{p} b_{k^{*}}^{2}$ and $p b_{p} v_{k^{*}} b_{k^{*}}$, which go to zero, so there is a good IR locality. Ultraviolet transfers will be bounded by $k^{*} b_{k^{*}} b_{q} v_{q}$. This quantity also goes to zero as $q$ increases, so there is an UV locality for this regime as well. Let us come up with bounds of relative locality. Indeed, the actual growth of magnetic energy was defined as $\varepsilon_{B}=\varepsilon-\varepsilon_{2}=C_{E} C_{K}^{-3 / 2} k v_{k}^{3}$. So, $p \ll k^{*}$ IR bound is $k^{*} C_{E}^{3 / 2} C_{K}^{-9 / 4}$ and UV bound is $k^{*} C_{E}^{-3 / 2} C_{M}^{9 / 4}$. We conclude that most of the interaction which result in magnetic energy growth must reside in the wavevector interval of $k^{*}\left[C_{E}^{3 / 2} C_{K}^{-9 / 4}, C_{E}^{-3 / 2} C_{M}^{9 / 4}\right]$. Numerically, if we substitute $C_{K}=1.6, C_{M}=4.2$, $C_{E}=0.05$ we get the interval of $k^{*}[0.004,2000]$. So, despite being asymptotically local, small-scale dynamo can be fairly nonlocal in practice.

Summarizing, the kinetic cascade at large scales and the MHD cascade at small scales are dominated by local interactions. The transition between the kinetic cascade and the MHD cascade is also dominated by local interactions, and since ideal MHD equations do not contain any scale explicitly, the efficiency of small-scale dynamo $C_{E}$ is a true universal constant. Note that $C_{E}$ relates energy fluxes, not en- 


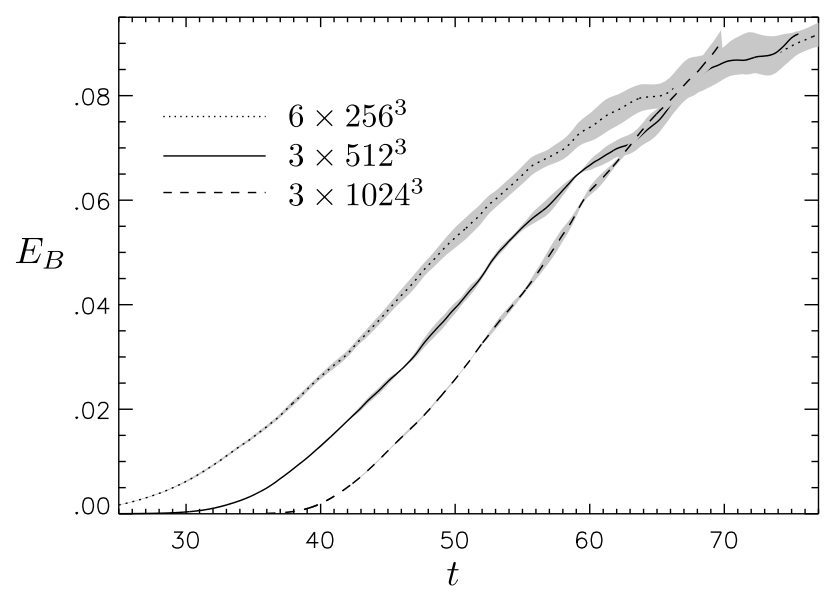

Fig. 2 Magnetic energy growth vs. time in code units, observed in simulations M1-6 $\left(\tau_{\eta}=0.091\right.$ in code units), M7-9 $\left(\tau_{\eta}=0.057\right)$ and M10-12 $\left(\tau_{\eta}=0.036\right)$. We used sample averages which greatly reduced fluctuations and allowed us to measure $C_{E}$ with sufficient precision.

ergies, so this claim is unaffected by the presence of intermittency. Magnetic spectrum at $k \ll k^{*}$ is dominated by nonlocal triads that reprocess magnetic energy from $k=k^{*}$ but, since this part of the spectrum contains negligible magnetic energy, our universality claim is unaffected by this nonlocality.

\subsection{Numerical Results}

We performed numerical simulations of statistically homogeneous isotropic smallscale dynamo by solving MHD equations with stochastic non-helical driving and explicit dissipation with $\operatorname{Pr}_{m}=1$. The details of the code and driving are described in detail in our earlier publications [36, 32] and Table 2 shows simulation parameters. We started each simulation from previously well-evolved driven hydro simulation by seeding low level white noise magnetic field. We ran several statistically independent simulations in each group and obtained growth rates and errors from sample averages. In all simulations, except M14, the energy injection rate was controlled. Fig 2 shows sample-averaged time evolution of magnetic energy. Growth is initially exponential and smoothly transition into the linear stage. Note, that scatter is initially small, but grows with time, which is consistent with the picture of magnetic field growing at progressively larger scales and having progressively less independent realizations in a single datacube. 


\subsection{On the Efficiency of Small-Scale Dynamo}

Our $C_{E}$ is much smaller than unity. One would expect a quantity of order unity because this is a universal number, determined only by strong interaction on equipartition scale. If we refer to the ideal incompressible MHD equations, written in terms of Elsässer variables, $\partial_{t} \mathbf{w}^{ \pm}+\hat{S}\left(\mathbf{w}^{\mp} \cdot \nabla\right) \mathbf{w}^{ \pm}=0$, the dynamo could be understood as decorrelation of $\mathbf{w}^{ \pm}$which are originally equal to each other in the hydrodynamic cascade. In our case this decorrelation is happening at the equipartition scale $1 / k^{*}$. Being time-dependent, it propagates upscale, while ordinarily energy cascade goes downscale. The small value of $C_{E}$ might be due to this. As opposed to picture with multiple reversals and dissipation due to microscopic diffusivity, typical for kinematic case, in our picture we appeal to turbulent diffusion which helps to create large-scale field. Both stretching and diffusion depend on turbulence at the same designated scale $1 / k^{*}$, so in the asymptotic regime of large Re one of these processes must dominate. As $C_{E}$ is small, stretching and diffusion are close to canceling each other.

\section{MHD Turbulence in the Inertial Range}

Inertial range of turbulence was introduced by [37] as a range of spatial scales where driving and dissipation are unimportant and perturbations exist due to energy transfer from one scale to another. In the inertial range of MHD turbulence perturbations of both velocity and magnetic field will be much smaller than the local Alfvénic velocity $v_{A}=B / \sqrt{4 \pi \rho}$, due to the turbulence spectrum being steeper than $k^{-1}$, therefore local mean magnetic field will strongly affect dynamics in this range [38, 39]. Furthermore, the large-scale dynamo we mentioned in Section 2 will operate in such objects as spiral galaxies and provide the mean field for the MHD turbulence in the interstellar medium (ISM).

As in the case of hydrodynamics, the study of MHD turbulence began with weakly compressible and incompressible cases which are directly applicable to many environments, such as stellar interiors, ICM and hot phases of the ISM. Later it was realized that many features of incompressible MHD turbulence are still present even in supersonic dynamics, due to the dominant effect of Alfvénic shearing [40, 41]. It had been pointed out by [30] that strong mean field incompressible turbulence is split into the cascade of Alfvénic mode, described by Reduced MHD or RMHD [42, 43] and the passive cascade of slow (pseudo-Alfvén) mode. In the strong mean field case it was sufficient to study only the Alfvénic dynamics, as it will determine all statistical properties of turbulence, such as spectrum or anisotropy. This decoupling was also observed in numerics. Luckily, being the limit of very strong mean field, RMHD has a two-parametric symmetry, which we will discuss further in Section 3.1, which, under certain conditions, makes universal cascade with power-law energy spectrum possible. 
Interaction of Alfvénic perturbations propagating in a strong mean field is unusual due to a peculiar dispersion relation of Alfvénic mode, $\omega=k_{\|} v_{A}$, where $k_{\|}$ is a wavevector parallel to the mean magnetic field. This results in a tendency of MHD turbulence to create "perpendicular cascade", where the flux of energy is preferentially directed perpendicular to the magnetic field. This tendency enhances the nonlinearity of the interaction, described by $\xi=\delta v k_{\perp} / v_{A} k_{\|}$, which is the ratio of the mean-field term to the nonlinear term, and results in development of essentially strong turbulence. As turbulence becomes marginally strong, $\xi \sim 1$, the cascading timescales become close to the dynamical timescales $\tau_{\text {casc }} \sim \tau_{\text {dyn }}=1 / w k_{\perp}$ and the perturbation frequency $\omega$ has a lower bound due to an uncertainty relation $\tau_{\text {casc }} \omega>1$ [30]. This makes turbulence being "stuck" in the $\xi \sim 1$ regime, which is known as "critical balance". There is another lower bound on $\omega$, due to the directional uncertainty of the $\mathbf{v}_{A}$, which was discovered in [44]. In the case of balanced MHD turbulence, which we consider in the next few sections, this two bounds coincide. We consider more general imbalanced case in Section 4.

Goldreich-Sridhar (1995, [30] henceforth GS95) model is predicting a $k^{-5 / 3}$ energy spectrum with anisotropy 1$]$ described as $k_{\|} \sim k_{\perp}^{2 / 3}$. Numerical studies [46, 47, [48] confirmed steep spectrum and scale-dependent anisotropy, but [47, 49] claimed a shallower than $-5 / 3$ spectral slope in the strong mean field case, which was close to $-3 / 2$. This motivated adjustments to the GS95 model [50, 51, 52]. A model with so called "dynamic alignment" [51, 53] became popular after the scale-dependent alignment was discovered in numerical simulations [54]. This model is based on the idea that the alignment between velocity and magnetic perturbations decreases the strength of the interaction scale-dependently, and claims that the alignment goes as $k^{-1 / 4}$. This would, as they argue, modify the spectral slope of MHD turbulence from the $-5 / 3$ Kolmogorov slope to the observed $-3 / 2$ slope. It also claims [53] that there is a self-consistent turbulent mechanism that produces such an alignment. Below we examine both the alignment and the spectrum.

\subsection{Basic Equations}

Ideal MHD equations describe the dynamics of ideally conducting inviscid fluid with magnetic field and can be written in Heaviside and $c=1$ units as

$$
\begin{array}{r}
\partial_{t} \rho+\nabla \cdot(\rho \mathbf{v})=0, \\
\rho\left(\partial_{t}+\mathbf{v} \cdot \nabla\right) \mathbf{v}=-\nabla P+\mathbf{j} \times \mathbf{B}, \\
\nabla \cdot \mathbf{B}=0, \\
\partial_{t} \mathbf{B}=\nabla \times(\mathbf{v} \times \mathbf{B}),
\end{array}
$$

\footnotetext{
${ }^{1}$ The anisotropy should be understood in terms of local magnetic field direction, i.e. the magnetic field direction at the given scale. The original treatment, e.g. the closure relations employed, in the Goldreich-Sridhar paper uses the global frame of reference which was noticed later in [45] and used in the numerical works that validated the theory [46 47 [4].
} 
with current $\mathbf{j}=\nabla \times \mathbf{B}$ and vorticity $\omega=\nabla \times \mathbf{v}$. This should be supplanted with energy equation and a prescription for pressure $P$. The incompressible limit assumes that the pressure is so high that the density is constant and velocity is purely solenoidal $(\nabla \cdot \mathbf{v}=0)$. This does not necessarily refer to the ratio of outer scale kinetic pressure to molecular pressure, but could be interpreted as scale-dependent condition. Indeed, if we go to the frame of the fluid, local perturbations of velocity will diminish with scale and will be much smaller than the speed of sound. In this situation it will be possible to decompose velocity into low-amplitude sonic waves and essentially incompressible component of $\mathbf{v}$, as long as we are not in the vicinity of a shock. The incompressible component, bound by $\nabla \cdot \mathbf{v}=0$, will be described by much simpler equations:

$$
\begin{array}{r}
\partial_{t} \mathbf{v}=\hat{S}(-\omega \times \mathbf{v}+\mathbf{j} \times \mathbf{b}), \\
\partial_{t} \mathbf{b}=\nabla \times(\mathbf{v} \times \mathbf{b}),
\end{array}
$$

where we renormalized magnetic field to velocity units $\mathbf{b}=\mathbf{B} / \rho^{1 / 2}$ (the absence of $4 \pi$ is due to Heaviside units) and used solenoidal projection operator $\hat{S}=(1-$ $\left.\nabla \Delta^{-1} \nabla\right)$ to get rid of pressure. Finally, in terms of Elsässer variables $\mathbf{w}^{ \pm}=\mathbf{v} \pm \mathbf{b}$ this could be rewritten as

$$
\partial_{t} \mathbf{w}^{ \pm}+\hat{S}\left(\mathbf{w}^{\mp} \cdot \nabla\right) \mathbf{w}^{ \pm}=0 .
$$

This equation resembles incompressible Euler's equation. Indeed, hydrodynamics is just a limit of $b=0$ in which $\mathbf{w}^{+}=\mathbf{w}^{-}$. This resemblance, however, is misleading, as the local mean magnetic field could not be excluded by the choice of reference frame and, as we noted earlier, will strongly affect dynamics on all scales. We can explicitly introduce local mean field as $\mathbf{v}_{\mathbf{A}}$, assuming that it is constant, so that $\delta \mathbf{w}^{ \pm}=\mathbf{w} \pm \mathbf{v}_{A}$ :

$$
\partial_{t} \delta \mathbf{w}^{ \pm} \mp\left(\mathbf{v}_{\mathbf{A}} \cdot \nabla\right) \delta \mathbf{w}^{ \pm}+\hat{S}\left(\delta \mathbf{w}^{\mp} \cdot \nabla\right) \delta \mathbf{w}^{ \pm}=0 .
$$

In the linear regime of small $\delta w$ 's they represent perturbations, propagating along and against the direction of the magnetic field, with nonlinear term describing their interaction. As we noted earlier, due to the resonance condition of Alfvénic perturbations they tend to create more perpendicular structure, making MHD turbulence progressively more anisotropic. This was empirically known from tokamak experiments and was used in so-called reduced MHD approximation, which neglected parallel gradients in the nonlinear term [42, 43]. Indeed, if we denote $\|$ and $\perp$ as directions parallel and perpendicular to $\mathbf{v}_{A}$, the mean field term $\left(v_{A} \nabla_{\|}\right) \delta w^{ \pm}$is much larger than $\left(\delta w_{\|}^{\mp} \nabla_{\|}\right) \delta w^{ \pm}$and the latter could be ignored in the inertial range where $\delta w^{ \pm} \ll v_{A}$. This will result in Equation 8 being split into

$$
\begin{aligned}
& \partial_{t} \delta \mathbf{w}_{\|}^{ \pm} \mp\left(\mathbf{v}_{\mathbf{A}} \cdot \nabla_{\|}\right) \delta \mathbf{w}_{\|}^{ \pm}+\hat{S}\left(\delta \mathbf{w}_{\perp}^{\mp} \cdot \nabla_{\perp}\right) \delta \mathbf{w}_{\|}^{ \pm}=0, \\
& \partial_{t} \delta \mathbf{w}_{\perp}^{ \pm} \mp\left(\mathbf{v}_{\mathbf{A}} \cdot \nabla_{\|}\right) \delta \mathbf{w}_{\perp}^{ \pm}+\hat{S}\left(\delta \mathbf{w}_{\perp}^{\mp} \cdot \nabla_{\perp}\right) \delta \mathbf{w}_{\perp}^{ \pm}=0,
\end{aligned}
$$


Table 3 Three-Dimensional RMHD Balanced Simulations

\begin{tabular}{lcccc}
\hline \hline Run & $n_{x} \cdot n_{y} \cdot n_{z}$ & Dissipation & $\langle\varepsilon\rangle$ & $L / \eta$ \\
\hline R1 & $256 \cdot 768^{2}$ & $-6.82 \cdot 10^{-14} k^{6}$ & 0.073 & 200 \\
R2 & $512 \cdot 1536^{2}$ & $-1.51 \cdot 10^{-15} k^{6}$ & 0.073 & 400 \\
R3 & $1024 \cdot 3072^{2}$ & $-3.33 \cdot 10^{-17} k^{6}$ & 0.073 & 800 \\
\hline R4 & $768^{3}$ & $-6.82 \cdot 10^{-14} k^{6}$ & 0.073 & 200 \\
R5 & $1536^{3}$ & $-1.51 \cdot 10^{-15} k^{6}$ & 0.073 & 400 \\
\hline R6 & $384 \cdot 1024^{2}$ & $-1.70 \cdot 10^{-4} k^{2}$ & 0.081 & 280 \\
R7 & $768 \cdot 2048^{2}$ & $-6.73 \cdot 10^{-5} k^{2}$ & 0.081 & 560 \\
\hline R8 & $768^{3}$ & $-1.26 \cdot 10^{-4} k^{2}$ & 0.073 & 350 \\
R9 & $1536^{3}$ & $-5.00 \cdot 10^{-5} k^{2}$ & 0.073 & 700 \\
\hline
\end{tabular}

which, physically represent a limit of very strong mean field where $\delta \mathbf{w}_{\|}^{ \pm}$is a slow (pseudo-Alfvén) mode and $\delta \mathbf{w}_{\perp}^{ \pm}$is the Alfvén mode and Equation 9 describes a passive dynamics of slow mode which is sheared by the Alfvén mode, while Equation 10 describes essentially nonlinear dynamics of the Alfvén mode and is known as reduced MHD. For our purposes, to figure out asymptotic behavior in the inertial range, it is sufficient to study Alfvénic dynamics and slow mode can be always added later, because it will have the same statistics.

It turns out that reduced MHD is often applicable beyond incompressible MHD limit, in a highly collisionless environments, such as tokamaks or the solar wind. This is due to the fact that Alfvén mode is transverse and does not require pressure support. Indeed, Alfvénic perturbations rely on magnetic tension as a restoring force and it is sufficient that charged particles be tied to magnetic field lines to provide inertia [55].

A remarkable property of RMHD is that it has a precise two-parametric symmetry: $\mathbf{w} \rightarrow \mathbf{w} A, \lambda \rightarrow \lambda B, t \rightarrow t B / A, \Lambda \rightarrow \Lambda B / A$. Here $\lambda$ is a perpendicular scale, $\Lambda$ is a parallel scale, $A$ and $B$ are arbitrary parameters of the transformation. This is similar to the symmetry in Euler equation $(\mathbf{B}=\mathbf{0}$ limit of MHD), except for a different prescription for parallel scale $\Lambda$ which now scales as time. It is due to this precise symmetry and the absence of any designated scale, that we can hypothesize universal regime, similar to hydrodynamic cascade of [37]. In nature, the universal regime for MHD can be achieved with $\delta w^{ \pm} \ll v_{A}$. In numerical simulations, we can directly solve RMHD equations, which have precise symmetry already built in. From practical viewpoint, the statistics from the full MHD simulation with $\delta w^{ \pm} \sim 0.1 v_{A}$ is virtually indistinguishable from RMHD statistics and even $\delta w^{ \pm} \sim v_{A}$ is still fairly similar to the strong mean field case [36].

\subsection{Basic Scalings in the Balanced Case}

As was shown in a rigorous perturbation study of weak MHD turbulence, it has a tendency of becoming stronger on smaller scales [56]. Indeed, if $k_{\|}$is constant and 
$k_{\perp}$ is increasing, $\xi=\delta w k_{\perp} / v_{A} k_{\|}$will increase, due to $\delta w \sim k_{\perp}^{-1 / 2}$ in this regime. This will naturally lead to strong turbulence, where $\xi$ will stuck around unity due to two competing processes: 1 ) increasing interaction by perpendicular cascade and 2) decrease of interaction due to the uncertainty relation $\tau_{\text {casc }} \omega>1$, where $\tau_{\text {casc }}$ is a cascading timescale. Therefore, MHD turbulence will be always marginally strong in the inertial range, which means that cascading timescale is associated with dynamical timescale $\tau_{\text {casc }} \sim \tau_{\mathrm{dyn}}=1 / \delta w k_{\perp}[30]$. In this case, assuming that energy transfer is local in scale and, therefore, depend only on perturbations amplitude on each scale, we can write Kolmogorov-type phenomenology as

$$
\varepsilon^{+}=\frac{\left(\delta w_{\lambda}^{+}\right)^{2} \delta w_{\lambda}^{-}}{\lambda}, \quad \varepsilon^{-}=\frac{\left(\delta w_{\lambda}^{-}\right)^{2} \delta w_{\lambda}^{+}}{\lambda}
$$

where $\varepsilon^{ \pm}$is an energy flux of each of the Elsässer variables and $\delta w_{\lambda}^{ \pm}$is a characteristic perturbation amplitude on a scale $\lambda$. Such an amplitude can be obtained by Fourier filtering with a dyadic filter in k-space, see, e.g., [29].

Since we consider so-called balanced case with both $w$ 's having the same statistical properties and energy fluxes, one of these equations is sufficient. This will result in a $\delta w \sim \lambda^{1 / 3}$, where $\lambda$ is a perpendicular scale, or, in terms of energy spectrum $E(k)$,

$$
E(k)=C_{K} \varepsilon^{2 / 3} k^{-5 / 3},
$$

where $C_{K}$ is known as Kolmogorov constant. We will be interested in Kolmogorov constant for MHD turbulence. This scaling is supposed to work until dissipation effects kick in. In our further numerical argumentation dissipation scale will play a big role, but not from a physical, but rather from a formal point of view. We will introduce an idealized scalar dissipation term in a RHS of Eq.7 $\mathrm{as}-v_{n}\left(-\nabla^{2}\right)^{n / 2} \mathbf{w}^{ \pm}$, where $n$ is an order of viscosity and $n=2$ correspond to normal Newtonian viscosity, while for $n>2$ it is called hyperviscosity. The dissipation scale for this GS95 model is the same as the one for Kolmogorov model, i.e. $\eta=\left(v_{n}^{3} / \varepsilon\right)^{1 /(3 n-2)}$. This is a unique combination of $v_{n}$ and $\varepsilon$ that has units of length. Note that Reynolds number, estimated as $v L / v_{2}$, where $L$ is an outer scale of turbulence, is around $(L / \eta)^{4 / 3}$.

Furthermore, the perturbations of $w$ will be strongly anisotropic and this anisotropy can be calculated from the critical balance condition $\xi \approx 1$, so that $k_{\|} \sim k_{\perp}^{2 / 3}$. Interestingly enough this could be obtained directly from units and the symmetry of RMHD equations from above. Indeed, in the RMHD limit, $k_{\|}$or $1 / \Lambda$ must be in a product with $v_{A}$, since only the product enters the original RMHD equations. We already assumed above that turbulence is local and each scale of turbulence has no knowledge of other scales, but only the local dissipation rate $\varepsilon$. In this case the only dimensionally correct combination for the parallel scale $\Lambda$, corresponding to perpendicular scale $\lambda$ is

$$
\Lambda=C_{A} v_{A} \lambda^{2 / 3} \varepsilon^{-1 / 3},
$$

where we introduced a dimensionless "anisotropy constant" $C_{A}$. Equations 12 and 13 roughly describe the spectrum and anisotropy of MHD turbulence. Note, that GS95's $-5 / 3$ is a basic scaling that should be corrected for intermittency. This cor- 

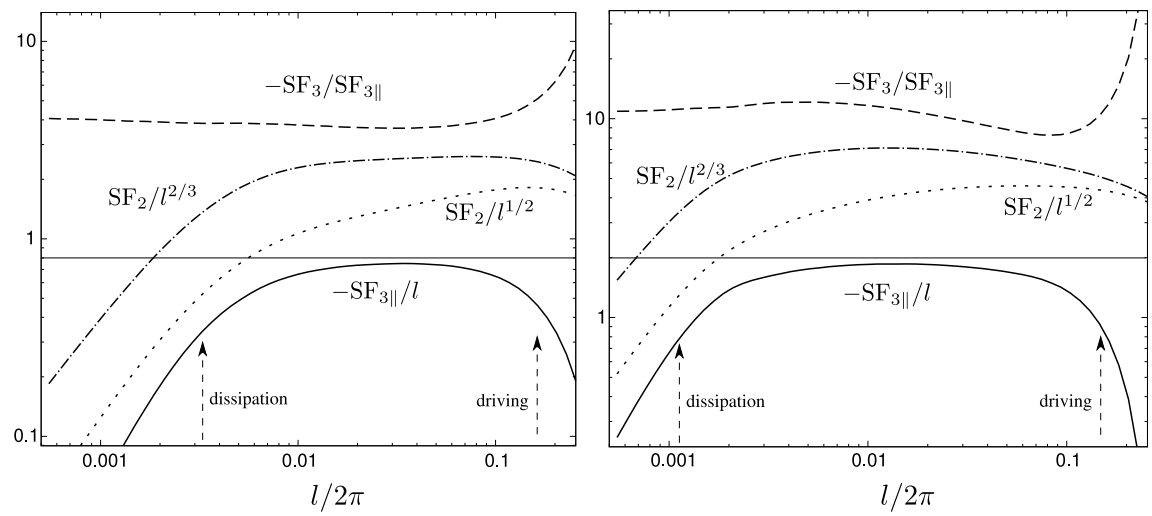

Fig. 3 Different structure functions vs the distance $l$, measured in hydrodynamic (left) and MHD (right) simulations. Solid lines show $-S F_{3 \|} / l \varepsilon$. The influence of driving and dissipation is minimized in the point where $-S F_{3 \|} / l \varepsilon$ is closer to its theoretical value. The dashed line indicates the ratio of the third order SF, defined in the text to the parallel third order SF. This ratio is a test for turbulence self-similarity, as long as this ratio is constant, the turbulence is well self-similar. Finally, dotted and dash-dotted lines indicate the same second order structure functions, compensated by $l^{1 / 2}$ and $l^{2 / 3}$ correspondingly, in arbitrary units. Here $l^{2 / 3}$ is the Richardson-Kolmogorov scaling and $l^{1 / 2}$ is the scaling that appears in Kraichnan DIA model for hydrodynamics, IroshnikovKraichnan model for MHD and B06 model.

rection is negative due to structure function power-law exponents being a concave function of their order [57] and is expected to be small in three-dimensional case. This correction for hydrodynamic turbulence is around -0.03 . Such a small deviation should be irrelevant in the context of debate between $-5 / 3$ and $-3 / 2$, which differ by about 0.17 .

A modification of the GS95 model was proposed by Boldyrev (2005, [51], 2006[53], henceforth B06) who suggested that the original GS95 scalings can be modified by a scale dependent factor that decreases the strength of the interaction, so that RHS of the Equation 11 is effectively multiplied by a factor of $(l / L)^{1 / 4}$, where $L$ is an outer scale. In this case the spectrum will be expressed as $E(k)=C_{K 2} \varepsilon^{2 / 3} k^{-3 / 2} L^{1 / 6}$. Note that this spectrum is the only dimensionally correct spectrum with $k^{-3 / 2}$ scaling, which does not contain dissipation scale $\eta$. The absence of $L / \eta$, is due to so-called zeroth law of turbulence which states that the amplitude at the outer scale should not depend on the viscosity. This law follows from the locality of energy transfer has been know empirically to hold very well. The dissipation scale of B06 model is different from that of the GS95 model and can be expressed as $\eta^{\prime}=\left(v_{n}^{3} / \varepsilon\right)^{1 /(3 n-1.5)} L^{0.5 /(3 n-1.5)}$. 


\subsection{Structure Functions and Spectra}

Structure and correlation functions (SF and CF) has been traditionally used in turbulence research for a long time. In theory these are quantities statistically averaged over ensemble, while in numerics the averaging is usually over time and volume using homogeneity and stationarity. The typical quantity people use in isotropic hydrodynamic turbulence is an isotropic second order structure function of velocity:

$$
S F^{2}(l)=\left\langle(v(\mathbf{r}-\mathbf{l})-v(\mathbf{r}))^{2}\right\rangle \mathbf{r}
$$

This is a difference in velocity between two points separated by vector $\mathbf{l}$, squared and averaged over the volume, i.e. the vector $\mathbf{r}$. This quantity could be represented by the sum of the "longitudinal" and "transverse" components with velocity decomposed into a direction perpendicular and parallel to $\mathbf{l}$. The longitudinal structure function is important in experimental research of hydrodynamic turbulence, since this is the primary quantity measured by the heated wire technique.

MHD turbulence is not isotropic, therefore, there is a wider variety of structure functions that one can possibly measure. However, in the RMHD limit there is a particular structure function which plays the similar role as the isotropic SF in hydrodynamics, the perpendicular SF

$$
S F_{\perp}^{2}(l)=\left\langle\left(w^{ \pm}(\mathbf{r}-l \mathbf{n})-w^{ \pm}(\mathbf{r})\right)^{2}\right\rangle_{\mathbf{r}}
$$

where $\mathbf{n}$ is a vector perpendicular to the magnetic field. Power spectra, on the other hand, are produced by obtaining a Fourier transform $\hat{\mathbf{v}}(\mathbf{k})$ of original quantity $\mathbf{v}(\mathbf{r})$ and taking the product $\frac{1}{2} \mathbf{v}_{\mathbf{i}}(\mathbf{k}) \mathbf{v}_{\mathbf{i}}^{*}(\mathbf{k})$, where $*$ is a complex conjugate. Relations between spectra and structure functions are well-known, see, e.g. [58].

A number of exact relations for structure functions are known both for hydro and MHD, see, e.g., [59]. The famous Kolmogorov -4/5 law relates a parallel signed structure function for velocity in the inertial range with the dissipation rate:

$$
S F_{3 \| h}(l)=\left\langle\left(\delta v_{l \|}\right)^{3}\right\rangle=-\frac{4}{5} \varepsilon l
$$

Another exact relation, similar to the Yaglom's -4/3 law for incompressible hydro exists for axially symmetric MHD turbulence:

$$
S F_{3 \|}(l)=\left\langle\delta w_{l \|}^{\mp}\left(\delta w_{l}^{ \pm}\right)^{2}\right\rangle=-2 \varepsilon l,
$$

where $l$ is taken perpendicular to the axis of statistical symmetry - the direction of the mean magnetic field $\mathbf{B}$ [60]. One can measure SFs above and argue about influence of dissipation and driving in each particular simulation. Fig. 3 shows several structure functions, compensated by various powers of $l$ and the ratio of parallel third order structure function and full third order SF, $S F_{3}=\left\langle|v(\mathbf{r}-\mathbf{l})-v(\mathbf{r})|^{3}\right\rangle$.

Normally, the inertial range in a simulation is defined as a range of scales where $-S F_{3 \|} / l$ is closest to its theoretical value, i.e. the influence of energy injection from 


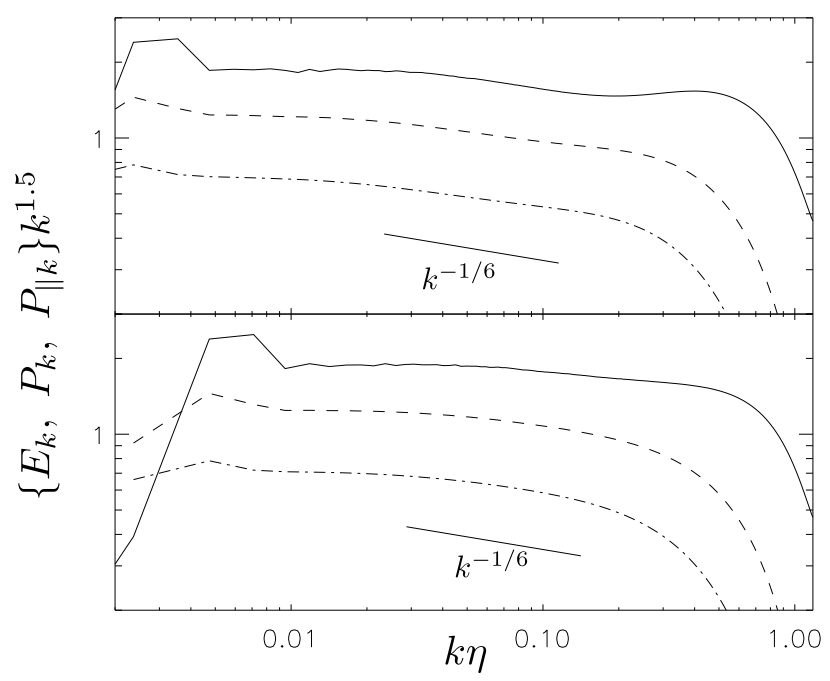

Fig. 4 Three types of spectra from a numerical simulations R1, R4. $E_{k}-$ solid, $P_{k}-$ dashed, $P_{\| k}-$ dash-dotted. In a simulation with limited resolution all three spectra have different shapes.

driving and energy dissipation from viscous term is minimized. Another test for the inertial range is the test for turbulence self-similarity, in particular one can take the above ratio of the unsigned and signed third order SFs. This ratio must be constant as long as turbulence is self-similar. Fig. 3 shows that hydrodynamic turbulence is rather self-similar and the scaling of the second-order structure function in the inertial range is around $l^{0.7}$, i.e. close to the Kolmogorov scaling. In the MHD simulation the self-similarity is broken and although one can argue that the scaling is closer to the $l^{2 / 3}$ in the point where $-S F_{3 \|} / l \varepsilon$ is closest to its theoretical value of 2 , claiming a certain scaling based on these data would be an overstatement. In the next section we will describe a rigorous method to claim a certain scaling based on numerical convergence in a series of simulations.

Power spectra are the measures, complimentary to second order structure functions. In particular, so-called one-dimensional power spectrum $P_{k}$ is a Fourier transform of the $S F_{2}$. This function is popular in the satellite measurements of the solar wind turbulence, where a particular quantity, $\mathbf{v}$ or $\mathbf{B}$ is measured as a function of time. It is then interpreted as an instant measurement along a line in a turbulent realization (so-called Taylor hypothesis). The power spectra from many samples like this are averaged to obtain $P_{k}$ for either velocity or magnetic field. Another experimental measure is the so-called parallel power spectrum $P_{\| k}$. It is obtained in the measurements of hydrodynamic turbulence by heated wire technique. A scalar quantity is measured in this technique, which is the velocity perturbation parallel to the average flow velocity. Similarly this is interpreted as a measurement in space by using Taylor frozen flow hypothesis. Finally, there is a power spectrum favored by numerics, which is a three-dimensional spectrum $E(k)$. This spectrum is obtained 
from full three-dimensional power spectrum $\frac{1}{2} \mathbf{v}(\mathbf{k}) \cdot \mathbf{v}^{*}(\mathbf{k})$ by integrating over the solid angle in $\mathbf{k}$ space, so that $E(k)$ is only a function of scalar $k$. In statistically isotropic hydro and MHD turbulence the integration is in spherical shells, while in RMHD, the parallel wavenumber is infinitely small compared to other wavenumbers, so the integration is, effectively, along all $k_{\|}$and the circle in $\mathbf{k}_{\perp}$ space, i.e. the isotropic spectrum is equivalent to the perpendicular spectrum. Three spectra $P(k)$, $P_{\|}(k)$ and $E(k)$ of the solenoidal vector field are related by the following expressions, see, e.g., [58]:

$$
\begin{gathered}
P(k)=\int_{k}^{\infty} E\left(k_{1}\right) \frac{d k_{1}}{k_{1}}, \\
P_{\|}(k)=\int_{k}^{\infty} E\left(k_{1}\right)\left(1-\frac{k^{2}}{k_{1}^{2}}\right) \frac{d k_{1}}{k_{1}},
\end{gathered}
$$

Fig. [4shows three types of spectra from the simulation. The primary spectrum was $E_{k}$ and the two other spectra were calculated by the above expressions. All three spectra have different shapes. If one would want to claim a particular scaling by qualitatively estimating the scaling from numerical spectrum, the estimate will depend on the type of the spectrum and the chosen range of $k$ used for fitting the scaling. Based on Fig. 4 4 one can claim any spectral slope between $-5 / 3$ and $-3 / 2$. This further reiterates the need of rigorous quantitative measurement based on numerical convergence, presented in the next section.

\subsection{The Numerical Scaling Argument}

As was noted before, turbulence with very long range of scales is common in astrophysics. Numerics, however, is not only unable to reproduce such range, but actually struggles to obtain any good "inertial range". In this situation a rigorous quantitative arguments have to be invented to investigate asymptotic scalings.

Suppose we performed several simulations with different Reynolds numbers. If we believe that turbulence is universal, and the scale separation between forcing scale and dissipation scale is large enough, the properties of small scales should not depend on how turbulence was driven and on the scale separation itself. This is because neither MHD nor hydrodynamic equations explicitly contain any scale, so simulation with a smaller dissipation scale could be considered, due to symmetry from above, as a simulation with the same dissipation scale, but larger driving scale. E.g., the small scale statistics in a $1024^{3}$ simulation should look the same as smallscale statistics in $512^{3}$, if the physical size of the elementary cell is the same and the dissipation scale is the same. Naturally, this scaling argument in numerics require that the geometry of the elementary cells are the same and the actual numerical scheme used to solve the equations is the same. Also, numerical equations should not contain any scale explicitly, but this is normally satisfied. What scaling argument does not require is a high precision on the dissipation scale or a particular form of dissipation, whether explicit or numerical. This is because we need that the statistics 


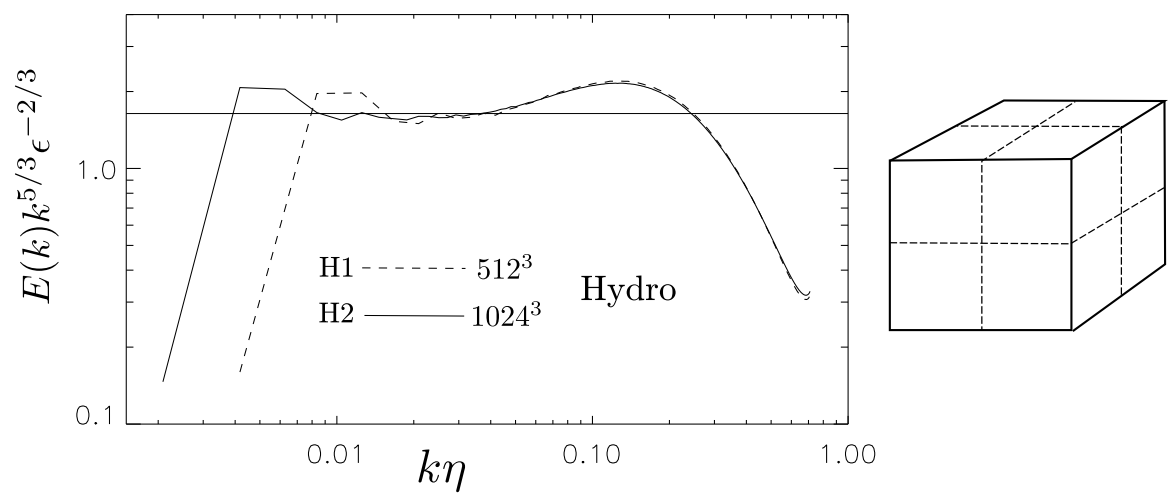

Fig. 5 The spectra from hydrodynamic simulations illustrate the numerical scaling argument. The large cube on the right can be split into smaller cubes with the same small scale statistics. Therefore as long as turbulence is scale-local and the effects of large scales could be neglected, the smaller simulation demonstrate the same statistics, as evident from convergence of dimensionless spectra on the left.

on small scales is similar in two simulations, which is the case when numerics is the same on dissipation scale and the influence of the outer scale is small by assumption of turbulence locality.

In practice the scaling argument or a resolution study is done in a following way: the averaged spectra in two simulations are expressed in dimensionless units corresponding to the expected scaling, for example a $E(k) k^{5 / 3} \varepsilon^{-2 / 3}$ is used for hydrodynamics, and plotted versus dimensionless wavenumber $k \eta$, where dissipation scale $\eta$ correspond to the same model, e.g. $\eta=\left(v^{3} / \varepsilon\right)^{1 / 4}$ is used for scalar second order viscosity $v$ and Kolmogorov phenomenology. Plotted this way the two spectra should collapse onto the same curve on the viscous scales, see, e.g., Fig. 5 This method has been used in hydrodynamics since long time ago, see, e.g., [61, 62, 63]. Although for hydrodynamics good convergence on the dissipation scale has been observed starting with rather moderate resolutions, which signifies that hydrodynamic cascade has good, narrow locality, the larger the resolution, the better the convergence should be. Note that in [63], which had very high resolution even the intermittency correction to the spectrum has been captured. So, the optimal strategy for MHD would be to perform the largest resolution simulations possible and do a resolution study with particular models in mind.

\subsection{Numerical Experiments}

We will briefly explain the numerical setup and methods used in [36, 32, 64, 31, 65]. For further detail the reader is referred to these publications. We used pseudospectral dealiased code to solve RMHD equations. Same code was used earlier for RMHD, incompressible MHD and incompressible hydrodynamic simulations. The RHS of 


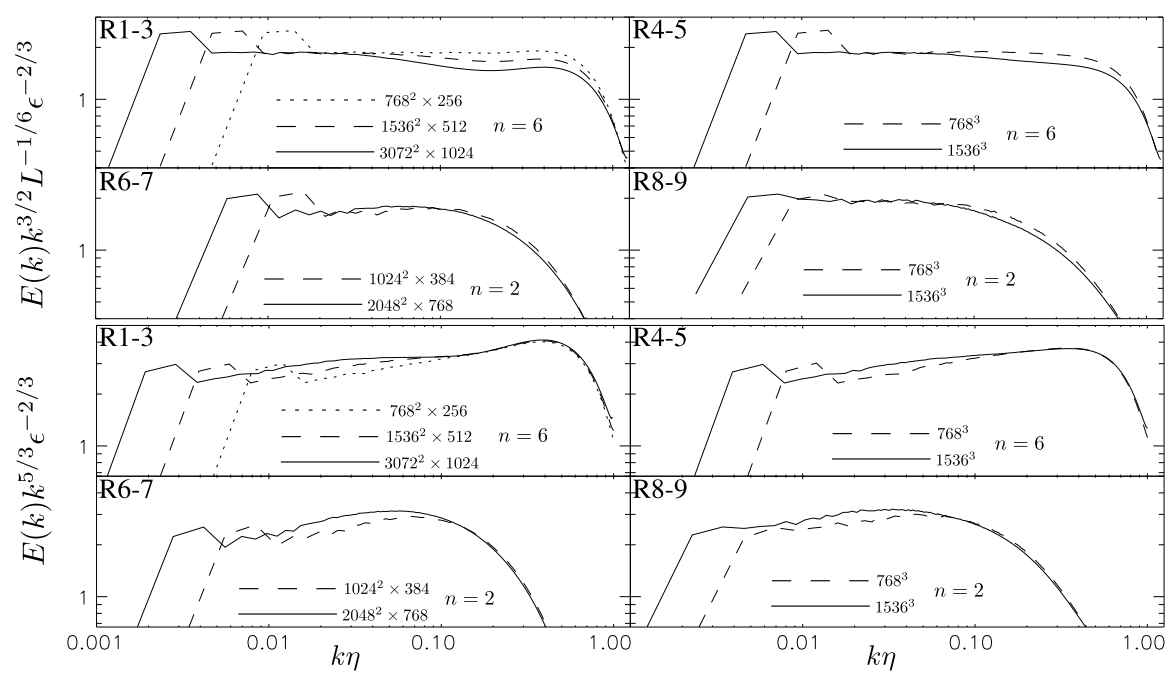

Fig. 6 Numerical convergence of spectra in all simulations. Two upper rows are used to study convergence assuming B06 model and two bottom rows - assuming GS95 model. Note that definition of dissipation scale $\eta$ depends on the model, this difference is tiny in hyperviscous simulations R1-5, but significant in viscous simulations R6-9. Numerical convergence require that spectra will be similar on small scales, including the dissipation scale, see, e.g. [62]. As we see from the plots, numerical convergence is absent for B06 model. For GS95 model the convergence is reached only at the dissipation scale. Higher-resolution simulations are required to demonstrate convergence in the inertial range.

Eq.10 was complemented by an explicit dissipation term $-v_{n}\left(-\nabla^{2}\right)^{n / 2} \mathbf{w}^{ \pm}$and forcing term $\mathbf{f}$. Diffusive terms with $n=2$ are referred to as normal viscosity and with $n>2$ are referred to as hyperviscosity. Table 3 shows the parameters of the balanced simulations. The Kolmogorov scale is defined as $\eta=\left(v_{n}^{3} / \varepsilon\right)^{1 /(3 n-2)}$, the integral scale $L=3 \pi / 4 E \int_{0}^{\infty} k^{-1} E(k) d k$ (which was approximately 0.79 for R1-3). Dimensionless ratio $L / \eta$ could serve as a "length of the spectrum", although spectrum is actually significantly shorter for $\mathrm{n}=2$ viscosity and somewhat shorter for $\mathrm{n}=6$ hyperviscosity.

Since we would like to use this review to illustrate the resolution study argument we used a variety of resolution, dissipation and driving schemes. There are four schemes, presented in Table 2 and used in simulations R1-3, R4-5, R6-7 and R89. In some of the simulations the resolution in the direction parallel to the mean magnetic field, $n_{x}$, was reduced by a factor compared to perpendicular resolution. This was deemed possible due to an empirically known lack of energy in the parallel direction in $k$-space and has been used before [49]. The R4-5 and R8-9 groups of simulations were fully resolved in parallel direction. One would expect that roughly the same resolution will be required in parallel and perpendicular direction [36]. In all simulation groups time step was strictly inversely proportional to the resolution, so that we can utilize the scaling argument. 
Driving had a constant energy injection rate for all simulations except R6-7, which had fully stochastic driving. All simulations except R8-9 had Elsässer driving, while R8-9 had velocity driving. All simulations were well-resolved and R6-7 were over resolved by a factor of 1.6 in scale (a factor of 2 in Re). The anisotropy of driving was that of a box, while injection rate was chosen so that the amplitude was around unity on outer scale, this roughly corresponds to critical balance on outer scale. Indeed, as we will show in subsequent section, since anisotropy constant is smaller than unity, our driving with $\lambda \sim \Lambda \sim 1$ and $\delta w \sim 1$ on outer scale is somewhat over-critical, so $\Lambda$ decreases after driving scale to satisfy uncertainty relation (see Fig. 96. This is good for maintaining critical balance over wide range of scales as it eliminates possibility for weak turbulence.

In presenting four groups of simulations, with different geometries of elementary cell, different dissipation terms and different driving, our intention is to show that the scaling argument works irrespective of numerical effects, but rather relies on scale separation and the assumption of universal scaling. Simulations R1-3 are the same as those presented in [31].

\subsection{Resolution Study for Balanced Spectra}

Fig. 6 presents a resolution study all simulations. The upper rows assume B06 scaling, while the bottom rows assume GS95 scaling. Reasonable convergence on small scales was achieved only for GS95 scaling. The normalized amplitude at the dissipation scale for two upper rows of plots systematically goes down with resolution, suggesting that $-3 / 2$ is not an asymptotic scaling. The flat part of the normalized spectrum on R1-3 plots was fit to obtain Kolmogorov constant of $C_{K A}=3.27 \pm 0.07$ which was reported in [31]. The total Kolmogorov constant for both Alfvén and slow mode in the above paper was estimated as $C_{K}=4.2 \pm 0.2$ for the case of isotropically driven turbulence with zero mean field, where the energy ratio of slow and Alfvén mode $C_{s}$ is between 1 and 1.3. This larger value $C_{K}=C_{K A}\left(1+C_{s}\right)^{1 / 3}$ is due to slow mode being passively advected and not contributing to nonlinearity. The measurement of $C_{K A}$ had relied on an assumption that the region around $k \eta \approx 0.07$ represent asymptotic regime. Recently, we performed simulations with resolution up to $4096^{3}$, which also confirmed the $-5 / 3$ spectrum [66]. Furthermore, it appears from these simulations that the residual energy, $E_{B}-E_{v}$ have the same spectral slope as the total energy, i.e. there is a constant fraction of residual energy in the inertial range. This fraction was measured in [66] to be around 0.15. Previously, the most popular model [49] suggested that the spectrum of the residual energy follows $k^{-2}$ scaling, which is problematic both conceptually and theoretically. We confirmed that the residual energy is a fraction of the total energy in the inertial range and made explanations suggesting different scalings for magnetic and kinetic energies unnecessary. 

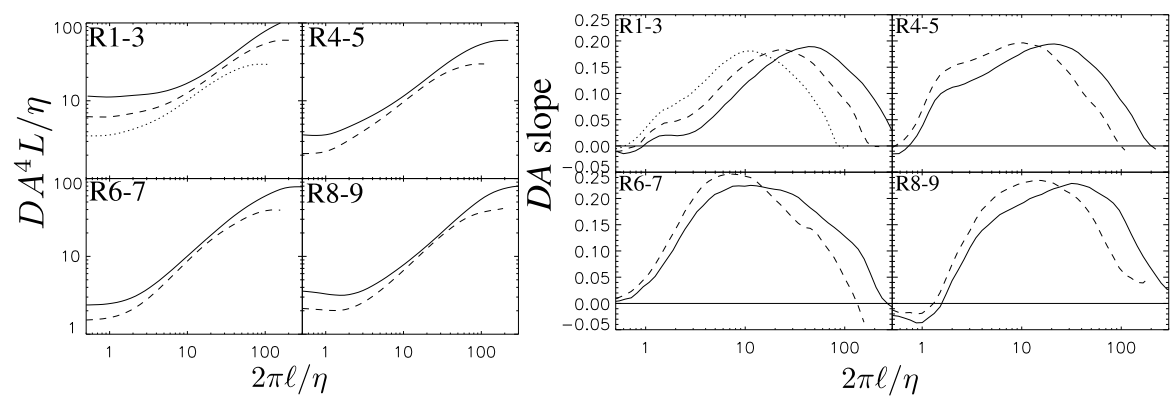

Fig. 7 Left: resolution study for "dynamic alignment", assuming B06 scaling. Both axis are dimensionless, solid is higher resolution and dashed is lower resolution. Convergence is absent for all simulations. This suggests that $l^{0.25}$ is not a universal scaling for alignment. Right: $D A$ slope, defined as $l / D A \partial D A / \partial l$, solid is higher resolution and dashed is lower resolution. Dynamic alignment slope does not converge and has a tendency of becoming smaller in higher-resolution simulations. This may indicate that the asymptotic alignment slope is zero, which will correspond to the GS95 model.

\subsection{Dynamic Alignment - Theories vs Measurements}

Recent simulations, as we discussed earlier, support GS95 model and therefore it can be considered correct in the zeroth approximation. However, we are far from believing that we understand all the effects of MHD turbulence. For instance, it is not clear how different alignment effects that we considered in $\$ 3.6$ may affect the basic properties of MHD turbulence at the limited range of scales when they exhibit scale-dependent properties.

An attempt to construct a model for such a behavior taking into account $D A$ was done in [51]. There it was proposed that $\mathbf{w}^{+}$and $\mathbf{w}^{-}$eddies are systematically aligned and therefore, GS95 model should be amended and the inertial range scaling should be modified. As we discussed earlier, this suggestion is not supported by either resolution studies or studies of the alignment/polarization effects that we performed. For instance, the original alignment idea was investigated numerically in in [54] and no significant alignment was found for the averaged angle between $\mathbf{w}^{+}$and $\mathbf{w}^{-}, A A=\left\langle\left|\delta \mathbf{w}_{\lambda}^{+} \times \delta \mathbf{w}_{\lambda}^{-}\right| /\left|\delta \mathbf{w}_{\lambda}^{+}\right|\left|\delta \mathbf{w}_{\lambda}^{-}\right|\right\rangle$, but when this angle was weighted with the amplitude $P I=\left\langle\left|\delta \mathbf{w}_{\lambda}^{+} \times \delta \mathbf{w}_{\lambda}^{-}\right|\right\rangle /\left\langle\left|\delta \mathbf{w}_{\lambda}^{+} \| \delta \mathbf{w}_{\lambda}^{-}\right|\right\rangle$, some alignment was found. Later [53] proposed the alignment between $\mathbf{v}$ and $\mathbf{b}$ and [67] suggested a particular amplitude-weighted measure, $D A=\left\langle\left|\delta \mathbf{v}_{\lambda} \times \delta \mathbf{b}_{\lambda}\right|\right\rangle /\left\langle\left|\delta \mathbf{v}_{\lambda}\right|\left|\delta \mathbf{b}_{\lambda}\right|\right\rangle$. We note that $D A$ is similar to PI but contain two effects: alignment and local imbalance. The latter could be measured with $I M=\left\langle\left|\delta\left(w_{\lambda}^{+}\right)^{2}-\delta\left(w_{\lambda}^{-}\right)^{2}\right|\right\rangle /\left\langle\delta\left(w_{\lambda}^{+}\right)^{2}+\delta\left(w_{\lambda}^{-}\right)^{2}\right\rangle$, [32].

In this section we check the assertion of [51,53] that alignment depends on scale as $\lambda^{1 / 4}$, by using $D A$ which is, by some reason, favored by aforementioned group. We did a resolution study of $D A$, assuming suggested scaling, which is presented on Fig. 7 Convergence was absent in all simulations. It appears that the claims of [53] were not substantiated by a proper resolution study. In general, a result from a single isolated simulation could be easily contaminated by the effects of outer scale, 
since it is not known a-priori how local MHD turbulence is and what resolution is sufficient to get rid of such effects. On the contrary, the resolution study offers a systematic approach to this problem.

Fig. 7 also shows "dynamic alignment" slope for all simulations. Although there similar to the previous plot there is no convergence, it is interesting to note that alignment slope decreases with resolution. This suggests that most likely the asymptotic state for the alignment slope is zero, i.e. alignment is scale-independent and GS95 model is recovered. Also, alignment from simulations R1-5 seems to indicate that the maximum of the alignment slope is tied to the outer scale, therefore alignment is a transitional effect.

In our earlier studies [54, 32] we measured several types of alignment and found no evidence that all alignment measures follow the same scaling, see, e.g., Fig. 8 . As one alignment measure, PI, has been already known to be well scale-dependent [54] prior to $D A$, it appears that a particular measure of the alignment in [67] was hand-picked for being most scale-dependent and no thorough explanation was given why it was preferred.

We are not aware of any convincing physical argumentation explaining why alignment should be a power-law of scale. [53] argues that alignment will tend to increase, but will be bounded by field wandering, i.e. the alignment on each scale will be created independently of other scales and will be proportional to the relative perturbation amplitude $\delta B / B$. But this violates two-parametric symmetry of RMHD equations mentioned above, which suggests that field wandering can not destroy alignment or imbalance. Indeed, a perfectly aligned state, e.g., with $\delta \mathbf{w}^{-}=0$ is a precise solution of MHD equations and it is not destroyed by its own field wandering. The alignment measured in simulations of strong MHD turbulence with different values of $\delta B_{L} / B_{0}$ showed very little or no dependence on this parameter [32].

Some alignment measures are scale-dependent over about one order of magnitude in scale. The origin of this scale-dependency was not yet clearly identified. However, the most plausible explanation is the combinations of two facts: a) MHD turbulence is less local than hydro turbulence [32, 64, 31] and b) the driving used in MHD turbulence does not particularly well reproduce the statistical properties of the inertial range. Thus transition to asymptotic statistics of the inertial range takes larger scale separation than in the hydrodynamic case.

The contribution to energy flux from different $k$ wavebands is important to understand, since most cascade models assume locality, or rather to say the very term "cascade" assumes locality. An analytical upper bound on locality suggests that the width of the energy transfer window can scale as $C_{K}^{9 / 4}$ [29], however, in practice turbulence can be more local. The observation of [32] that MHD simulations normally lack bottleneck effect, even with high-order dissipation, while hydrodynamic simulations always have bottleneck, which is especially dramatic with high-order dissipation, is consistent with above conjecture on locality, since bottleneck effect relies on locality of energy transfer. As locality constraint depends on the efficiency of the energy transfer, so that the efficient energy transfer must be local, while inefficient one could be nonlocal [64, 31, 29]. As we observe larger $C_{K}$ in MHD tur- 


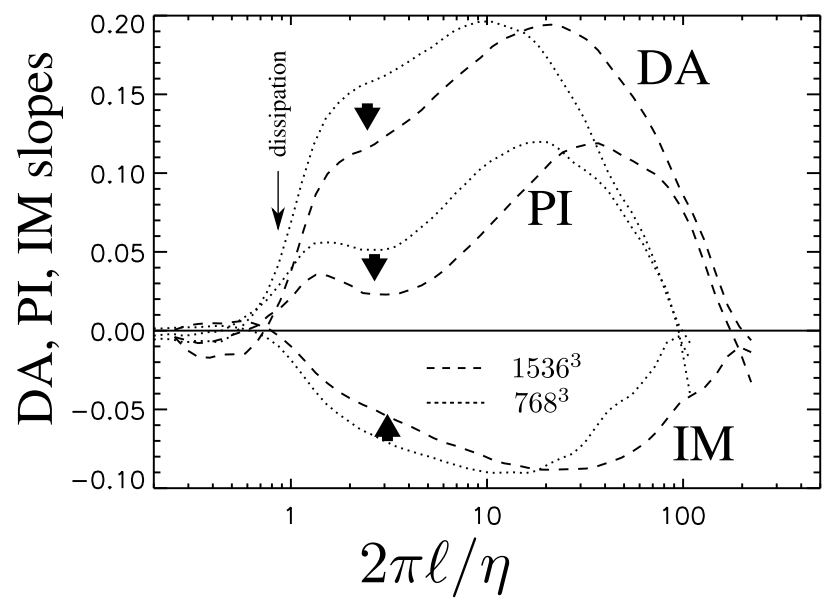

Fig. 8 Slopes of several alignment measures vs scale in R4-5 (for definitions see the text). Each measure follows its own scaling, however there are indications that they are all tied to the outer scale, due to the maximum of alignment being a fraction of the outer scale, which is an indication that their scale-dependency is of transient nature.

bulence compared to hydrodynamic turbulence, the former could be less local than the latter, which is consistent with our earlier findings.

\subsection{Dynamic Alignment - Relation to Spectra}

The papers [53, 67] and subsequent papers assert that the particular measure of alignment, $D A$ in our notation, is weakening interaction scale-dependently, so that the energy spectral slope is modified. In particular, the above papers claim that if $D A \sim \lambda^{\alpha}$, then the spectrum $E(k) \sim k^{-5 / 3+2 / 3 \alpha}$ which, in the case of $\alpha=1 / 4$ will result in $E(k) \sim k^{-3 / 2}$. Numerics does not show flat spectra if one compensates $E(k)$ slope with $2 / 3$ of $D A$ slope, however. Let us critically examine the claim $E(k) \sim k^{-5 / 3+2 / 3 \alpha}$ from the theoretical viewpoint. The exact relation describing energy flux through scales is given by Eq.17. Let us analyze this statistical average for a "+" component at a particular value of $l:\left\langle\delta w_{l \|}^{-}\left(\delta w_{l}^{+}\right)^{2}\right\rangle$. Indeed, it appears that the anti-correlation of $\delta w_{l}^{-}$and $\delta w_{l}^{+}$could result in a reduction of the above statistical average, as [53] seems to allege. There are three arguments against this, however.

Firstly, the $D A$ does not describe such an anti-correlation, and something different, such as $I M$ should be taken instead. So, the assumption of the interaction weakening rely on the claim that alignment measures scale similarly. As we see from Fig. 8, the slopes of $D A$ and $I M$ are quite different and if $D A$ reaches the maximum slope of 0.2 , the $I M$ only reaches the the maximum slope of 0.09 and this value does not increase with resolution. This is far from 0.25, required in B06 model. 
The numerical analysis of [67] and subsequent papers, however, dealt exclusively with $D A$ and the earlier publication [54] that reported several different alignment measures, which scaled differently, was ignored and the strong claim of interaction weakening was made nevertheless. However, with present numerics reported so far, even assuming an anti-correlation argument, one can not deduce that the interaction is weakened by a factor of $l^{1 / 4}$.

Secondly, the $D A$ is based on a second order measure, while $\left\langle\delta w_{l\|\|}^{-}\left(\delta w_{l}^{+}\right)^{2}\right\rangle$ is third-order. We also know that $A A$ which is based on zeroth order (sin of the alignment angle) is very weakly scale-dependent [54], we can extrapolate to "third-order alignment" having $\sim \lambda^{3 \alpha / 2}$ dependence and the spectral slope will be $E(k) \sim k^{-5 / 3+\alpha}$. This is actually more numerically consistent with the data than $E(k) \sim k^{-5 / 3+2 / 3 \alpha}$, because $\alpha$ is typically below 0.2 and the spectral slope is often flatter than $-3 / 2$ close to the driving scale.

Thirdly, and most importantly, there is no rigorous argumentation that could suggest that the discussed anti-correlation necessarily reduces the above statistical average. Indeed, the $\delta w_{l \|}^{-}$is a signed quantity, and so is the whole expression under the statistical average. Therefore, the value of the statistical average is not necessarily related to the RMS value of the expression, but rather depend on the skewness of the PDF of the expression. This is most obviously indicated by the Fig. 3] where the ratio of unsigned to signed statistical average is about 10. In fact, this ratio could be arbitrarily large, e.g. in weak MHD turbulence, where taking larger $B_{0}$ will result in decreased energy rates, the above PDF becoming closer to Gaussian and its skewness going to zero. It is only the GS95 similarity hypothesis for the case of strong MHD turbulence, which is similar to Kolmogorov hypothesis, that asserts that the skewness is independent on scale, allows us to derive the $k^{-5 / 3}$ spectrum. When one wants to explore a different similarity relations, as [51, 53] did, it is necessary to argue in favor of the scale-independent skewness again. In MHD turbulence, which has fluctuations of the imbalance ratios, it is not clear what self-similarity prescription should be adopted. In a more detailed treatment of the imbalanced turbulence below we argue that is it very likely that the skewness of $\delta w_{l \|}^{-}\left(\delta w_{l}^{+}\right)^{2}$ and $\delta w_{l \|}^{+}\left(\delta w_{l}^{-}\right)^{2}$ could be very different in the imbalanced case, due to the fact that the stronger component is cascaded weakly, i.e. $\left\langle\delta w_{l\|\|}^{-}\left(\delta w_{l}^{+}\right)^{2}\right\rangle$ is not the constant fraction of $\left\langle\left|\delta w_{l}^{-}\right|\left(\delta w_{l}^{+}\right)^{2}\right\rangle$, but could be much smaller.

To summarize, the assertion that the interaction is weakened by the $D A$ factor is at best heuristic and could be seriously questioned by both numerical data and theoretical argumentation. Apart from this, we reiterate the arguments of previous sections that the numerical evidence strongly suggests that $D A$ and other alignment measures become constant in the inertial range and that the asymptotic inertial-range scaling for MHD turbulence is closer to $-5 / 3$. 


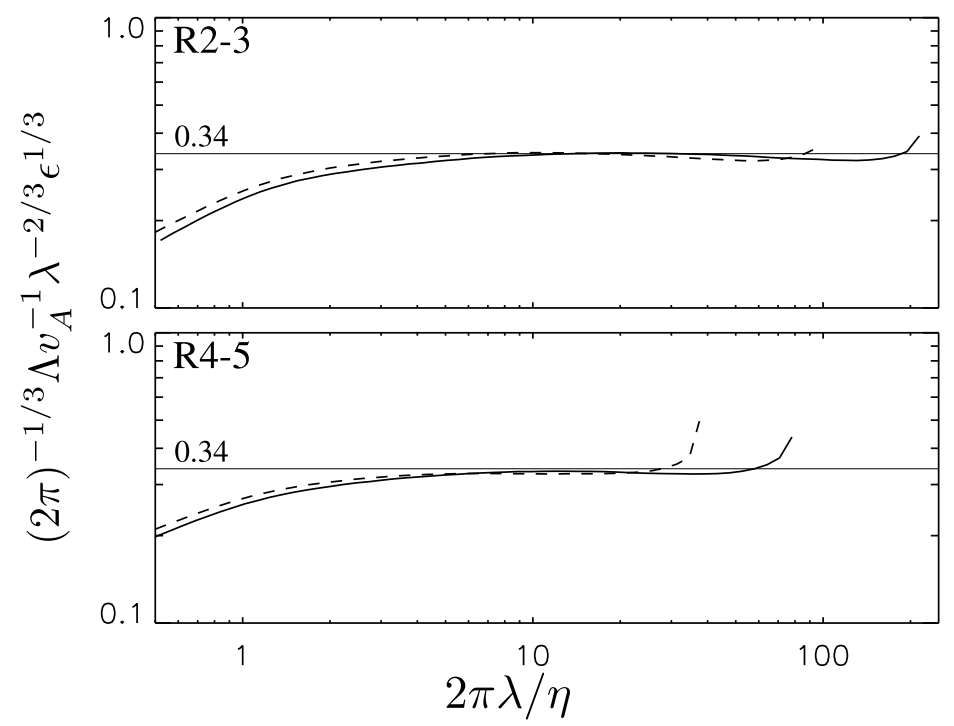

Fig. 9 The scaling study for anisotropy shows moderately good convergence to a universal anisotropy $\Lambda=C_{A} v_{A} \lambda^{2 / 3} \varepsilon^{-1 / 3}$ with anisotropy constant $C_{A}$ of around 0.63 .

\subsection{Anisotropy - Balanced case}

In Section 3 we suggested that anisotropy should be universal in the inertial range and expressed as $\Lambda=C_{A} v_{A} \lambda^{2 / 3} \varepsilon^{-1 / 3}$, where $C_{A}$ is an anisotropy constant to be determined from the numerical experiment or observation. Note, that both Alfvénic and slow modes should have the same anisotropy. This is because they have the same ratio of propagation to nonlinear timescales. Fig.9 shows anisotropy for the two best resolved groups R1-3 and R4-5. We used a model independent method of minimum parallel structure function, described in detail in [36]. Alternative definitions of local mean field give comparable results, as long as they are reasonable. From R1-3 we obtain $C_{A}=0.63$. Note, that the conventional definition of critical balance involve the amplitude, rather than $(\varepsilon \lambda)^{1 / 3}$, so the constant in this classical formulation will be $C_{A} C_{K}^{1 / 2} \approx 1.1$, which is closer to unity. Together with energy spectrum this is a full description of universal axisymmetric two-dimensional spectrum of MHD turbulence in the inertial range.

\subsection{Basic Properties of Balanced MHD turbulence}

In this review we argue that the properties of Alfvén and slow components of MHD turbulence in the inertial range will be determined only by the Alfvén speed $v_{A}$, 
dissipation rate $\varepsilon$ and the scale of interest $\lambda$. The energy spectrum and anisotropy of Alfvén mode will be expressed as

$$
\begin{gathered}
E(k)=C_{K} \varepsilon^{2 / 3} k^{-5 / 3}, \\
\Lambda / \lambda=C_{A} v_{A}(\lambda \varepsilon)^{-1 / 3},
\end{gathered}
$$

with $C_{K}=3.3$ and $C_{A}=0.63$. If the slow mode is present, its anisotropy will be the same, and it will contribute to both energy and dissipation rate. Assuming the ratio of slow to Alfvén energies between 1 and 1.3, the latter was observed in statistically isotropic high resolution MHD simulation with no mean field, we can use $C_{K}=4.2$ for the total energy spectrum [31].

Anisotropy of MHD turbulence is an important property that affects such processes as interaction with cosmic rays, see, e.g., [68]. Since cosmic ray pressure in our Galaxy is of the same order as dynamic pressure, their importance should not be underestimated. Another process affected is the three-dimensional turbulent reconnection, see, e.g., [45].

Previous measurements of the energy slope relied on the highest-resolution simulation and fitted the slope in the fixed $k$-range close to the driving scale, typically between $k=5$ and $k=20$. We argue that such a fit is unphysical unless a numerical convergence has been demonstrated. We can plot the spectrum vs dimensionless $k \eta$ and if we clearly see a converged dissipation range and a bottleneck range, we can assume that larger scales, in terms of $k \eta$ represent inertial range. In fitting fixed $k$ range at low $k$ we will never get rid of the influence of the driving scale. In fitting a fixed $k \eta$ range, the effects of the driving will diminish with increasing resolution.

Since we still have trouble transitioning into the inertial range in large mean field simulations, for now it is impossible to demonstrate inertial range in statistically isotropic simulations similar to once presented in [49]. This is because we do not expect a universal power-law scaling in transAlfvénic regime, due to the absence of appropriate symmetries and the transitioning to subAlfvénic regime, where such scaling is possible, will require some extra scale separation. These two transitions require numerical resolution that is even higher than the highest resolution presented in this paper and for now seem computationally impossible.

Full compressible MHD equations contain extra degrees of freedom, which, in a weakly compressible case, entails the additional cascade of the fast MHD mode, possibly of weak nature. Supersonic simulations with moderate Mach numbers [40] show that Alfvénic cascade is pretty resilient and is not much affected by compressible motions. The models of the "universal" supersonic turbulence covering supersonic large scales and effectively subsonic small scales are based mainly on simulations with limited resolution and unlikely to hold true. This is further reinforced by the results of this paper which demonstrated that even a much simpler case of sub-Alfvénic turbulence require fairly high resolutions to obtain an asymptotic scaling. 


\section{Imbalanced MHD turbulence}

While hydrodynamic turbulence have only one energy cascade, the incompressible MHD turbulence has two, due to the exact conservation of the Elsässer (oppositely going wave packets') "energies". This can be also formulated as the conservation of total energy and cross-helicity2. The situation of zero total cross-helicity, which we considered in previous sections has been called "balanced" turbulence as the amount of oppositely moving wavepackets balance each other, the alternative being "imbalanced" turbulence. Most of the above studies concentrated on the balanced case, and, without exception, the GS95 model, which is the strong cascading model with critical balance, can only be kept self-consistent assuming balanced case.

The real MHD turbulence, however, is often imbalanced, such as in situations when the mean magnetic field is present and we have a strong localized source of perturbations. The perfect example is the solar wind, where satellite measurements discovered strong correlations between $\mathbf{v}$ and $\mathbf{B}$ since long time ago. These correlations actually correspond to the imbalanced turbulence with the dominant component propagating away from the Sun. If the mean magnetic field of the Parker spiral is directed locally outwards the Sun then the dominant component will be $\mathbf{w}^{-}$, otherwise it'll be $\mathbf{w}^{+}$.

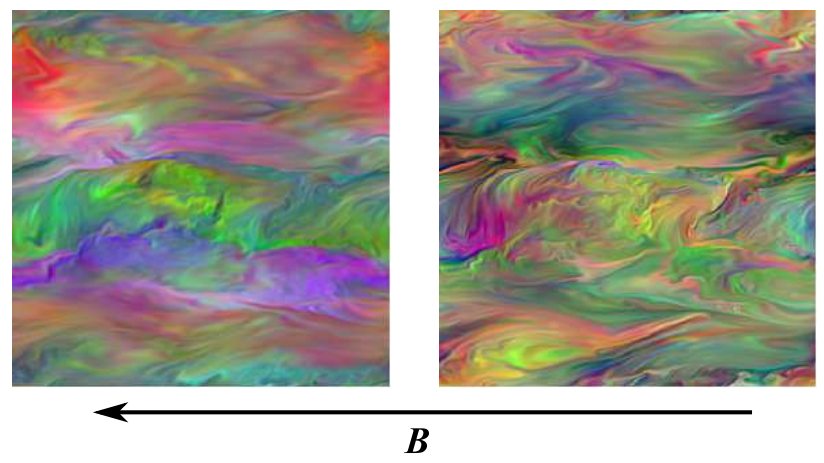

Fig. 10 The slices of $\mathbf{w}^{+}=\mathbf{v}+\mathbf{B} / \sqrt{4 \pi \rho}$ (left) and $\mathbf{w}^{-}=\mathbf{v}-\mathbf{B} / \sqrt{4 \pi \rho}$ (right) from the threedimensional MHD simulation with strong mean magnetic field and imbalance.

Certainly, we expect similar phenomena happen in the active galactic nuclei (AGN), where the jet has a strong large mean magnetic field component and the perturbations will propagate primarily away from the central engine, where they will be excited by either Blandford-Znajek mechanism, for the inside jet, or by the motions of the magnetic field footpoints, embedded into the turbulent accretion disk. Another example is the interstellar medium (ISM) turbulence in spiral galaxies. Indeed, in spiral galaxies, due to the action of the large-scale dynamo there is a large-scale

\footnotetext{
${ }^{2}$ The latter, $\int \mathbf{v} \cdot \mathbf{B} d^{3} x$ is a quantity conserved in the absence of dissipation.
} 
component of the magnetic field, spanning the radius of the disk itself. The ISM turbulence, however, is inhomogeneous, due to the energy sources for turbulence (supernovas and stellar winds) distributed unevenly in the disk. This will create imbalanced turbulence, which might properties different from the balanced one, which has implications for ISM heating, cosmic ray propagation and many other physical processes in the ISM.

Finally, from the theoretical viewpoint, it is impossible to fully understand balanced turbulence by itself, if the more general imbalanced case is not treated. This is due to the fact that turbulence is a stochastic phenomena with all quantities fluctuating and every piece of turbulence at any given time can have imbalance in it. In this respect, while the mean-field Kolmogorov model can be expanded to include fluctuations of the dissipation rate in the volume, the mean field GS95 model can not.

Imbalanced turbulence, or "turbulence with non-zero cross-helicity" has been discussed long ago by a number of authors [69, 70, 71, 72]. This work testified that the non-zero cross-helicity modifies the turbulence. Although these studies correctly reproduced separate cascades for energy and cross-helicity, they were based on then-popular models of MHD turbulence and later it became evident that these are problematic. For example, the closure theory of isotropic MHD turbulence [73], which reproduced Iroshnikov-Kraichnan model can be criticized on the basis that the ad-hoc term for "relaxation of triple correlations", happen to be larger than real physical nonlinear interaction and makes MHD turbulence, effectively, isotropic. Numerics, however, show that strong MHD turbulence is locally anisotropic, as we demonstrated in previous sections. Another class of models were based on so-called two-dimensional MHD turbulence that, as we demonstrated in previous sections, is unable to reproduce basic properties of the real three-dimensional turbulence, such as strong interaction with critical balance.

\subsection{Theoretical considerations}

As we explain in the previous sections, the MHD cascade is primarily perpendicular and as it proceeds to small scales, the applicability of weak interaction breaks down, and Alfvénic turbulence becomes strong. In this situation GS95 assumed that the frequency of the wavepacket can not be smaller than the inverse lifetime of the wavepacket, estimated from nonlinear interaction. In the GS95 closure model there is an explicit ad-hoc term that allows for the increase of the wave frequency. Unlike previous models this term is scale-dependent and is based on the assumption of turbulence locality, i.e. that there is one characteristic amplitude of perturbation pertaining to each scale and that this perturbation determines the strength of the interaction and finally renormalization of frequencies. However, as was realized as early as in the original GS95 paper in the imbalanced case we have two charac- 
teristic amplitudes, $w^{+}, w^{-}$, and the choice for frequency renormalization becomes uncleat . Any theory of strong imbalanced turbulence, must deal with this difficulty.

Let us first demonstrate that a straightforward generalization of GS95 for the imbalanced case does not work. If we assume that the frequency renormalization for one wavepacket is determined by the shear rate of the oppositely moving wavepacket, the wave with small amplitude (say, $w^{-}$) may only weakly perturb large amplitude wave $w^{+}$and the frequency of cascaded $w^{+}$will conserve. On the other hand, $w^{+}$may strongly perturb $w^{-}$and $w^{-}$'s frequency will be determined as $w_{l}^{+} / 4$. This mismatch in frequencies creates an inconsistency in the paradigm of scalelocal cascade where both wavepackets must have both parallel and perpendicular wavenumbers comparable. As the cascade proceeds to small scales this mismatch only increases, making the cascade nonlocal and inefficient. Such shutdown of the cascade on small scales is unacceptable, since in the stationary case it must carry a constant energy flux for both components. In order to deal with this fundamental difficulty, one must assume something extra to the original GS95 critical balance.

Currently there were several propositions how to deal with strong anisotropic imbalanced MHD turbulence. In [74], the authors proposed that the parallel scale for both components is determined by the shear rate of the stronger component. This model predicts the same anisotropy for both components. In [44] the authors proposed a new formulation for critical balance for the stronger component. In [75] an advection-diffusion model of cascading was adopted, where advection was describing perpendicular cascade and diffusion was describing the increase of frequencies. These three models clearly state the difficulty described above and try to resolve it with the new physical argumentation that goes beyond the original GS95 critical balance. These three models smoothly transition to the balanced theory of GS95 in the limit of small imbalance. Several other models has been suggested, advocating a different picture, in particular the influence of so-called dynamic alignment. In [76] the authors argued that the dynamic alignment will effectively lead to the same nonlinear timescale for both components. This has been criticized as grossly inconsistent with numerics [36, 64] and having no meaningful physical limit for large imbalances.

\subsection{Lithwick, Goldreich \& Sridhar (2007) model, LGS07}

LGS07 argue that the strong wave $w^{+}$is also cascaded strongly and its frequency is equal to the frequency of the weak wave, i.e. the critical balance for strong wave uses

\footnotetext{
${ }^{3}$ We assume that imbalanced turbulence is "strong" as long as the applicability of weak Alfvénic turbulence breaks down. This requires that at least one component is perturbed strongly. In the imbalanced turbulence the amplitude of the dominant component is larger, so that in the transition to strong regime the applicability of weak cascading of the subdominant component breaks down first.

${ }^{4}$ Throughout this paper we assume that $w^{+}$is the larger-amplitude wave. This choice, however, is purely arbitrary and corresponds to the choice of positive versus negative total cross-helicity.
} 
the amplitude of the strong wave itself $\left(w^{+} \Lambda=v_{A} \lambda\right)$. In this case the anisotropies of the waves are identical. The formulas for energy cascading are strong cascading formulas, i.e.

$$
\varepsilon^{\mp}=\frac{\left(w^{\mp}(\lambda)\right)^{2} w^{ \pm}(\lambda)}{\lambda} .
$$

This lead to the prediction $w^{+} / w^{-}=\varepsilon^{+} / \varepsilon^{-}$. In terms of energy spectra the model predicts

$$
E_{k}^{ \pm}=C_{K}\left(\varepsilon^{ \pm}\right)^{4 / 3}\left(\varepsilon^{\mp}\right)^{-2 / 3} k^{5 / 3},
$$

where the Kolmogorov constant $C_{K}$ must be the same for the theory to have a limit of standard balanced MHD turbulence.

\subsection{Beresnyak \& Lazarian (2008) model, BL08}

BL08a relaxes the assumption of local cascading for the strong component $w^{+}$, while saying the $w^{-}$is cascaded in a GS95-like way. In BL08a picture the waves have different anisotropies (see Fig. 11b and the $w^{+}$wave actually have smaller anisotropy than $w^{-}$, which is opposite to what a naive application of critical balance would predict. The anisotropies of the waves are determined by

$$
\begin{aligned}
& w^{+}\left(\lambda_{1}\right) \Lambda^{-}\left(\lambda_{1}\right)=v_{A} \lambda_{1}, \\
& w^{+}\left(\lambda_{2}\right) \Lambda^{+}\left(\lambda^{*}\right)=v_{A} \lambda_{1},
\end{aligned}
$$

where $\lambda^{*}=\sqrt{\lambda_{1} \lambda_{2}}$, and the energy cascading is determined by weak cascading of the dominant wave and strong cascading of the subdominant wave:

$$
\begin{gathered}
\varepsilon^{+}=\frac{\left(w^{+}\left(\lambda_{2}\right)\right)^{2} w^{-}\left(\lambda_{1}\right)}{\lambda_{1}} \cdot \frac{w^{-}\left(\lambda_{1}\right) \Lambda^{-}\left(\lambda_{1}\right)}{{ }_{A} \lambda_{1}} \cdot f\left(\lambda_{1} / \lambda_{2}\right), \\
\varepsilon^{-}=\frac{\left(w^{-}\left(\lambda_{1}\right)\right)^{2} w^{+}\left(\lambda_{1}\right)}{\lambda_{1}} .
\end{gathered}
$$

One of the interesting properties of BL08a model is that, unlike LGS07 and C08, it does not produce self-similar (power-law) solutions when turbulence is driven with the same anisotropy for $w^{+}$and $w^{-}$on the outer scale. BL08a, however, claim that, on sufficiently small scales, the initial non-power-law solution will transit into asymptotic power law solution that has $\Lambda_{0}^{-} / \Lambda_{0}^{+}=\varepsilon^{+} / \varepsilon^{-}$and $\lambda_{2} / \lambda_{1}=\left(\varepsilon^{+} / \varepsilon^{-}\right)^{3 / 2}$. The range of scales for the transition region was not specified by BL08a, but it was assumed that larger imbalance will require larger transition region. 


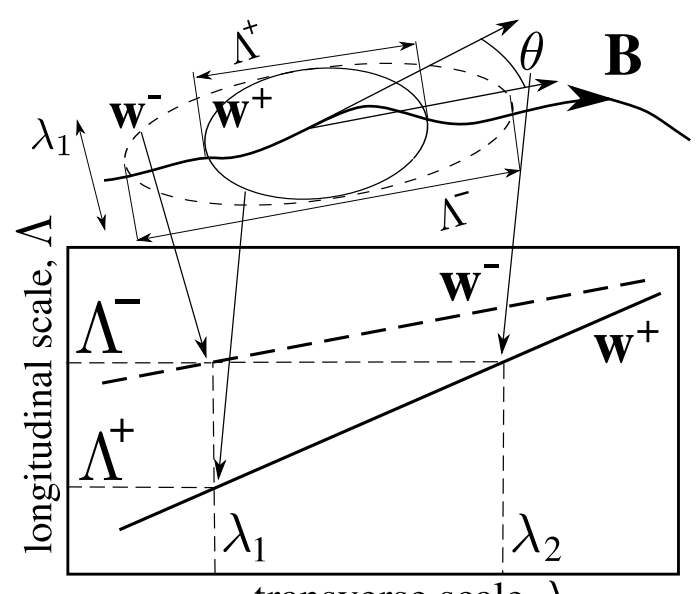

transverse scale, $\lambda$

Fig. 11 Upper: a $\mathbf{w}^{+}$wavepacket, produced by cascading by $\mathbf{w}^{-}$wavepacket is aligned with respect to $\mathbf{w}^{-}$wavepacket, but misaligned with respect to the local mean field on scale $\lambda_{1}$, by the angle $\theta$. Lower: the longitudinal scale $\Lambda$ of the wavepackets, as a function of their transverse scale, $\lambda ; \Lambda^{+}, \Lambda^{-}, \lambda_{1}, \lambda_{2}$ are the notations used in this paper. From Beresnyak \& Lazarian model [44].

Table 4 Three-Dimensional RMHD Imbalanced Simulations

\begin{tabular}{lccccr}
\hline \hline Run & Resolution & $f$ & Dissipation & $\varepsilon^{+} / \varepsilon^{-}$ & $\left(w^{+}\right)^{2} /\left(w^{-}\right)^{2}$ \\
\hline I1 & $512 \cdot 1024^{2}$ & $w^{ \pm}$ & $-1.9 \cdot 10^{-4} k^{2}$ & 1.187 & $1.35 \pm 0.04$ \\
I2 & $768^{3}$ & $w^{ \pm}$ & $-6.8 \cdot 10^{-14} k^{6}$ & 1.187 & $1.42 \pm 0.04$ \\
I3 & $512 \cdot 1024^{2}$ & $w^{ \pm}$ & $-1.9 \cdot 10^{-4} k^{2}$ & 1.412 & $1.88 \pm 0.04$ \\
I4 & $768^{3}$ & $w^{ \pm}$ & $-6.8 \cdot 10^{-14} k^{6}$ & 1.412 & $1.98 \pm 0.03$ \\
I5 & $1024 \cdot 1536^{2}$ & $w^{ \pm}$ & $-1.5 \cdot 10^{-15} k^{6}$ & 2 & $5.57 \pm 0.08$ \\
I6 & $1024 \cdot 1536^{2}$ & $w^{ \pm}$ & $-1.5 \cdot 10^{-15} k^{6}$ & 4.5 & $45.2 \pm 1.5$ \\
\hline
\end{tabular}

\subsection{Perez \& Boldyrev (2009) model, PB09}

Unlike the models described above PB09 employs dynamic alignment which decreases without limit to smaller scales as $l^{1 / 4}$ and claims the $3 / 2$ spectral slope for both components. In this respect it is similar to [51, 53]. It does, however, a big step beyond these papers by claiming that alignment will effectively result in the same nonlinear timescales for both components, which effectively lead to $\left(w^{+}\right)^{2} /\left(w^{-}\right)^{2}=\varepsilon^{+} / \varepsilon^{-}$. It could be rephrased that PB09 predicts turbulent viscosity which is equal for both components. It is not clear, however, how this could be made consistent with the limit of large imbalances, where the weak component will not be able to produce any sizable turbulent viscosity. 


\subsection{Imbalanced simulations}

Table 4 summarizes our high-resolution experiments with imbalanced driving. All experiments were conducted to reproduce stationary turbulence. We started our high resolution simulations with earlier lower-resolution runs that were evolved for a long time, typically hundreds Alfvénic times and reached stationary state. The imbalanced runs were evolved for longer times, up to 40 dynamical times, due to longer cascading timescales for the stronger component. The energy injection rates were kept constant in I1-6 and the fluctuating dissipation rate was within few percent of the former.

\subsection{Nonlinear cascading and dissipation rate}

Compared to spectral slopes, dissipation rates are robust quantities that require much smaller dynamical range and resolution to converge. Fig. 12 shows energy imbalance $\left(w^{+}\right)^{2} /\left(w^{-}\right)^{2}$ versus dissipation rate imbalance $\varepsilon^{+} / \varepsilon^{-}$for simulations I2, I4, I5 and I6. We also use two data points from our earlier simulations with large imbalances, A7 and A5 from BL09a. I1 and I3 are simulations with normal viscosity similar to I2 and I4. They show slightly less energy imbalances than I2 and I4. We see that most data points are above the prediction of LGS07, which is consistent with BL08. In other words, numerics strongly suggest that

$$
\frac{\left(w^{+}\right)^{2}}{\left(w^{-}\right)^{2}} \geq\left(\frac{\varepsilon^{+}}{\varepsilon^{-}}\right)^{2} .
$$

Although there is a tentative correspondence between LGS07 and the data for small degrees of imbalance, the deviations for large imbalances are significant. The important lesson, however, that in the case of small imbalances the cascading smoothly transition to the balanced case, i.e. the prediction of GS95 model. This is an important verification that the exactly balanced case is not a special case, in a sense.

In the case of strong imbalance it suggests that the strong component cascading rate is smaller than what is expected from strong cascading. As to PB09 prediction, it is inconsistent with data for all degrees of imbalance including those with small imbalance and normal viscosity, i.e. I1 and I3.

\subsection{Imbalanced Spectra}

Fig. 13 shows spectra from low-imbalance simulation I2, compensated by the predictions of PB09 and LGS07. We see that the collapse of two curves for $w^{+}$and $w^{-}$ is much better for the LGS07 model, however the spectral slope is much closer to 

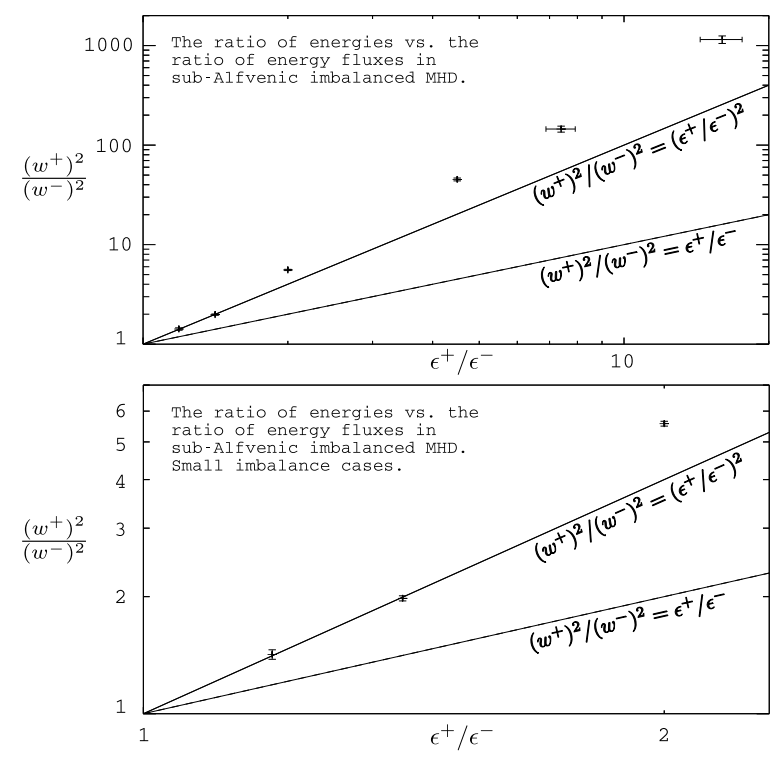

Fig. 12 Energy imbalances versus dissipation rate imbalance. Lower panel shows a magnified portion of the upper panel. Solid line: LGS07 prediction, dashed line: a formula from PB09, this also is a prediction for purely viscous dissipation. The point indicate measurements from simulations, where errorbars indicate fluctuation in time. I1 and I3 are simulations with normal viscosity which have slightly lower energy imbalance than I 2 and I4. This is an indication that in these simulations viscosity was affecting outer scales. Two high imbalance points are taken from [36]. For a fixed dissipation ratio the energy imbalance has a tendency to only increase with resolution.

$-3 / 2$ than to $-5 / 3$. The issue of spectral slope was discussed in previous section with respect to the balanced simulations. We were arguing that MHD cascade is less local than hydro cascade and is being influenced by driving on a larger range of scales, more importantly the statistical properties of driving is different than asymptotic regime of MHD cascade, which results in a transition range of scales of about one order of magnitude. We expect the same effect to operate in the imbalanced case. Indeed, if we neglect the part of the spectrum with $\mathrm{k}$ between 2 and 20, the spectrum could be considered flat on the lower panel of Fig. 13 In this deviation of spectral slope from $-5 / 3$ we do not see any significant differences between the balanced case, which was discussed extensively in the previous sections and the low-imbalance case.

Fig. 14 shows spectra from all I1-6 simulations, compensated by the prediction of LGS07. For lower imbalances the collapse is reasonably good and become progressively worse for larger imbalances. This deviation, however, does not fully follow the prediction of the asymptotic power-law solutions from BL08, which will predict that the solid curve will go above $C_{K A}$ and the dashed curve - below it. This is possibly explained by the fact that asymptotic power law solutions were not reached 

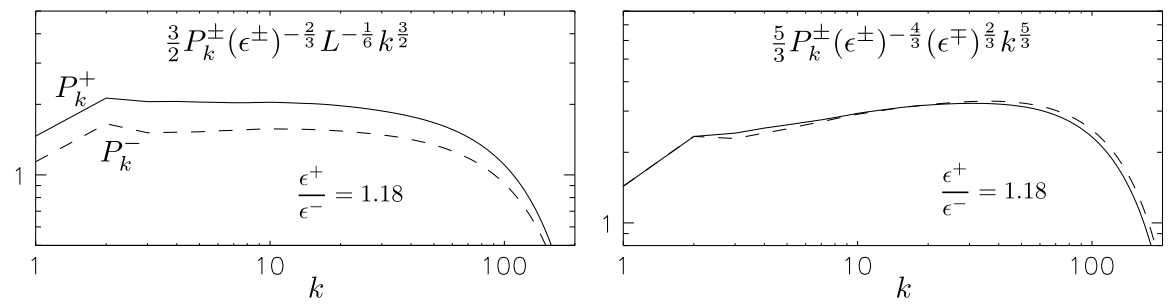

Fig. 13 Energy spectra for $w^{+}$(solid) and $w^{-}$(dashed) from simulation I2, compensated by factors that correspond to PB09 (upper panel) and LGS07 (lower panel). Either theory is confirmed for this low-imbalanced case if the spectra for $w^{+}$and $w^{-}$collapse onto the same curve. We see that the collapse is much better for the LGS07 model.

in these limited resolution experiments, this is also observed for anisotropies which we consider in the next section.

\subsection{Imbalanced Anisotropies}

We measured parallel and perpendicular structure functions in simulations I1-I6 in order to quantify anisotropies of eddies. The perpendicular structure function was defined above. In the RMHD case which physically correspond to the case of very strong mean field the perpendicular structure function must be calculated with respect to the global mean field. The same is not true for the parallel structure function. Indeed, measuring parallel SF with respect to the global field will destroy scaledependent anisotropy, even in the case of very strong field. If we have $\delta B_{L} / B_{0} \ll 1$, the field line wandering will be of the order of $B_{0} / \delta B_{L}$, while the GS95 anisotropy on the scale $l$ will be much higher, $\sim B_{0} / \delta B_{l}$, by a factor of $B_{L} / B_{l}$. The direction of the mean field will deviate from the direction of the local field by the angle which is much larger than the angle of GS95 anisotropy. This will result in an incorrect estimation of the parallel structure function which will be contaminated by contribution from perpendicular direction. Therefore, one must measure anisotropy with respect to local mean field, as was realized in [46, 48, 32].

For the parallel structure function we will use the model-independent method suggested in [32] or "minimum method", namely

$$
S F_{\|}^{2}(\Lambda)=\min _{\lambda}\left\langle\left(w^{ \pm}\left(\mathbf{r}-\Lambda \mathbf{b}_{\lambda} / b_{\lambda}\right)-w^{ \pm}(\mathbf{r})\right)^{2}\right\rangle_{\mathbf{r}}
$$

Where $\mathbf{b}_{\lambda}$ is the magnetic field smoothed on scale $\lambda$ with Gaussian kernel. It turns out that this method gives very close results to the previously suggested methods of choosing the local mean field, most prominently in the balanced case. We choose this method as it does not contain any arbitrary assumptions as previous methods. 


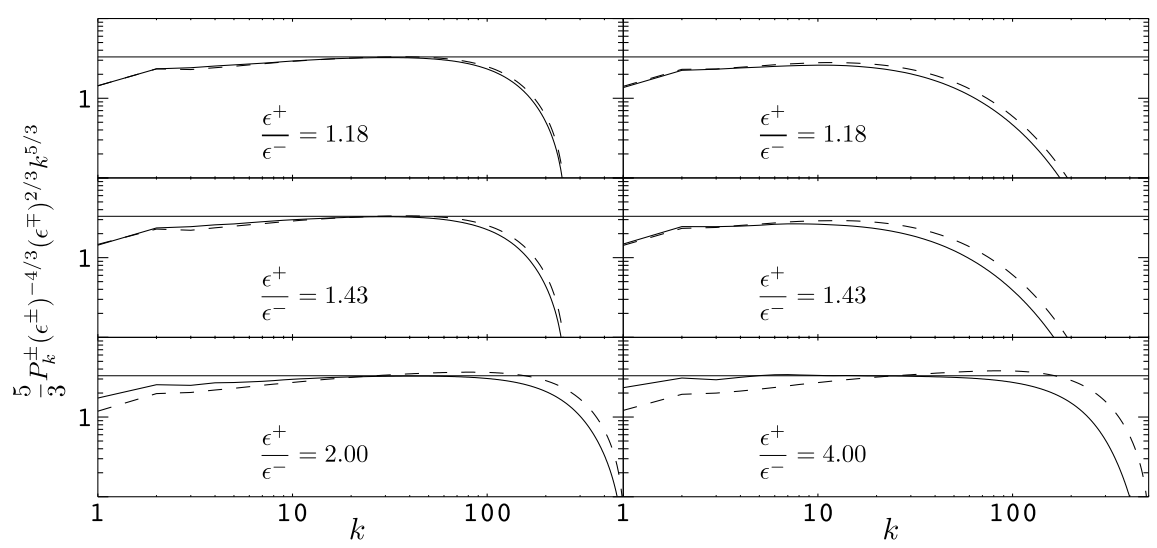

Fig. 14 Energy spectra for $w^{+}$(solid) and $w^{-}$(dashed) for simulations I1-I6, compensated by factors that correspond to LGS07. The thin solid line corresponds to Kolmogorov constant for Alfvénic turbulence $C_{K A}=3.27$. The factor $5 / 3$ is introduced due to the difference between $P_{k}$ and $E_{k}$.

As long as we know both parallel and perpendicular structure functions, the mapping $\Lambda(\lambda)$ is obtained from the equation $S F_{\|}^{2}\left(w^{ \pm}, \Lambda\right)=S F_{\perp}^{2}\left(w^{ \pm}, \lambda\right)$. Physically this correspond to measurement of the parallel eddy size $\Lambda$, whose energy is concentrated on scales $\lambda$.

Fig. 15 shows anisotropies for I1-6 simulations. All simulations were driven by the same anisotropies on the outer scale, which is unfavorable for obtaining the asymptotic power law solutions of BL08, which have an anisotropy ratio which is constant through scales and equal to $\varepsilon^{+} / \varepsilon^{-}$. It is, however, favorable to the LGS07 model, which predicts the same $w^{+}$and $w^{-}$anisotropies for all scales. Therefore, these simulations are a sensitive test between LGS07 and BL08 models, both of which are roughly consistent in terms of energy ratios and spectra for small imbalances. If LGS07 was true, starting with the same anisotropies on outer scale, this should be preserved by the cascade on smaller scales, but this is not what is observed on Fig. 15, where anisotropies start to diverge on smaller scales. The ratio of anisotropies is roughly consistent with BL08 asymptotic power-law solutions for small imbalances and falls short for larger imbalances. This is explained by the fact that it is harder to get to the asymptotic power-law solutions for larger imbalances, as was also observed for the case of power spectra.

\section{Compressibility in MHD turbulence}

Our discussion so far was centered at the incompressible MHD turbulence. From the astrophysical point of view compressibility is an essential property that cannot be ignored. This calls for studies to what extend our earlier description survives in 


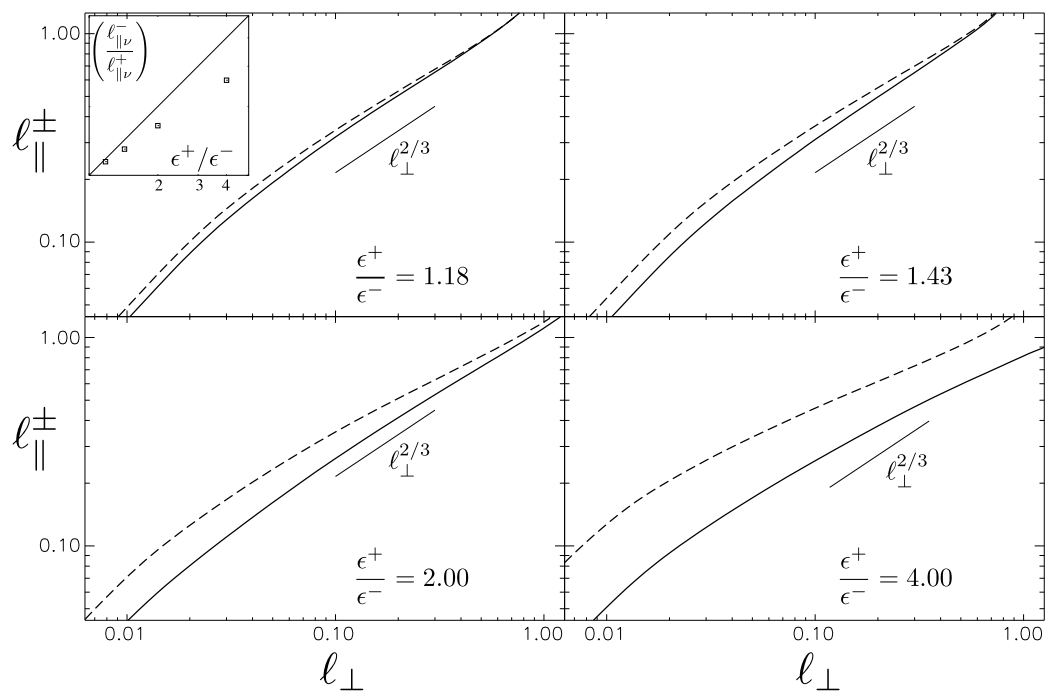

Fig. 15 Anisotropies for $w^{+}$(solid) and $w^{-}$(dashed), simulations I1-I6. The relation between parallel scale $\Lambda$ and perpendicular scale $\lambda$ is obtained by second order structure functions, as explained in the text. The small upper inset shows the ratio of anisotropies on smallest scales vs the prediction of BL08 for the asymptotic power-law solution, which is $\varepsilon^{+} / \varepsilon^{-}$.

realistic set ups and what additional properties are gained by compressible MHD turbulence.

Kolmogorov turbulence is known to be applicable to compressible non-magnetized fluids and therefore one should expect that some properties of GS95 model should persist at least for low Mach number magnetic turbulence. At the same time, new modes are excited in MHD in the presence of compressibility. In particular, if MHD turbulence in the incompressible limit can be decomposed into Alfvén and pseudoAlfvén modes, in the case of compressible MHD turbulence, three modes, namely, Alfvén, slow and fast are present. While the pseudo-Alfvén modes are a limiting case of the slow modes for compressibility going to zero, the fast modes present a new type of motion intrinsic for compressible media 5 .

\subsection{Decomposition into fundamental modes}

The original procedure of decomposition of MHD simulations into different modes was proposed by Cho \& Lazarian ([77, 40] henceforth CL02, CL03, respectively). Unlike earlier discussions which dealt with small perturbations the aforementioned

${ }^{5}$ In the limiting case of compressibility going to zero, the fast modes are sound waves with phase speed going to infinity. 


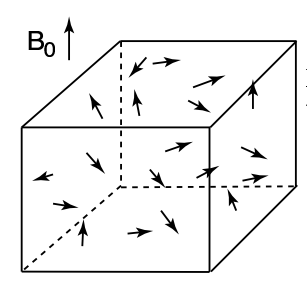

real space

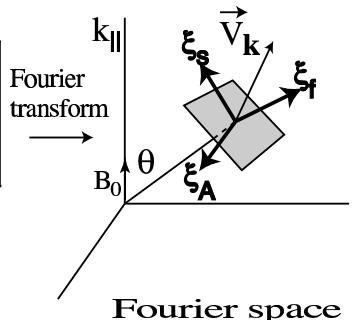

Fourier space

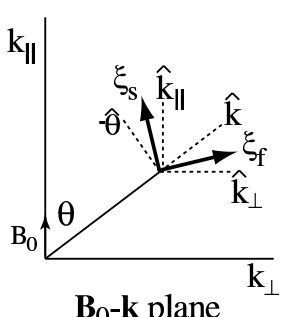

$\mathbf{B}_{0}-\mathbf{k}$ plane

Fig. 16 Graphical representation of the mode separation method. We separate the Alfvén, slow and fast modes by the projection of the velocity Fourier component $\mathbf{v}_{k}$ on the bases $\hat{\xi}_{A}$, $\hat{\xi}_{s}$ and $\hat{\xi}_{f}$, respectively. From CL03

papers demonstrated the decomposition of the transAlfvénic turbulence, i.e. the turbulence with substantial amplitudes. The procedure of decomposition is performed in the Fourier space by a simple projection of the velocity Fourier components $\hat{\mathbf{u}}$ on the direction of the displacement vector for each mode (see Fig. 16). The directions of the displacement vectors $\hat{\xi}_{s}, \hat{\xi}_{f}$, and $\hat{\xi}_{A}$ corresponding to the slow mode, fast and Alfvén modes, respectively, are defined by their unit vectors

$$
\begin{gathered}
\hat{\xi}_{s} \propto(-1+\alpha-\sqrt{D}) k_{\|} \hat{\mathbf{k}}_{\|}+(1+\alpha-\sqrt{D}) k_{\perp} \hat{\mathbf{k}}_{\perp}, \\
\hat{\xi}_{f} \propto(-1+\alpha+\sqrt{D}) k_{\|} \hat{\mathbf{k}}_{\|}+(1+\alpha+\sqrt{D}) k_{\perp} \hat{\mathbf{k}}_{\perp}, \\
\hat{\xi}_{A}=-\hat{\varphi}=\hat{\mathbf{k}}_{\perp} \times \hat{\mathbf{k}}_{\|},
\end{gathered}
$$

where $\mathbf{k}_{\|}$and $\mathbf{k}_{\perp}$ are the parallel and perpendicular to $\mathbf{B}_{\text {ext }}$ components of wave vector, respectively, $D=(1+\alpha)^{2}-4 \alpha \cos ^{2} \theta, \alpha=a^{2} / V_{A}^{2}, \theta$ is the angle between $\mathbf{k}$ and $\mathbf{B}_{\text {ext }}$, and $\hat{\varphi}$ is the azimuthal basis in the spherical polar coordinate system. The Fourier components of each mode can be directly used to calculate spectra. For other measures, such as structure functions, transforms back to the real space were used.

The results of CL02 and CL03 revealed several important properties of MHD turbulence. For the cases studied, they revealed that GS95 scaling is valid for Alfvén modes:

$$
\text { Alfvén: } E^{A}(k) \propto k^{-5 / 3}, \quad k_{\|} \propto k_{\perp}^{2 / 3} .
$$

Slow modes also follows the GS95 model for both high $\beta$ and mildly supersonic low $\beta$ cases:

$$
\text { Slow: } E^{s}(k) \propto k^{-5 / 3}, \quad k_{\|} \propto k_{\perp}^{2 / 3} .
$$

For the highly supersonic low $\beta$ case, the kinetic energy spectrum of slow modes tends to be steeper, which may be related to the formation of shocks.

Fast mode spectra are compatible with acoustic turbulence scaling relations:

Fast: $E^{f}(k) \propto k^{-3 / 2}$, isotropic spectrum. 

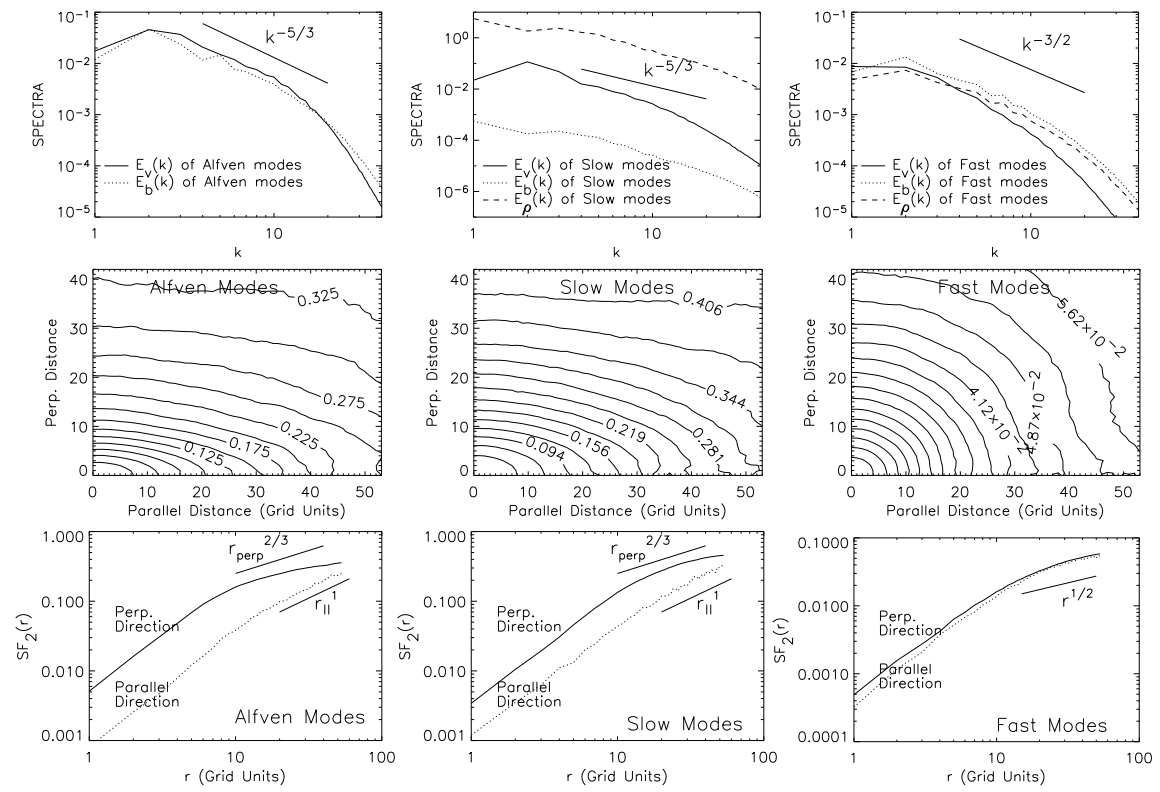

Fig. 17 Highly supersonic low $\beta\left(\beta \sim 0.02\right.$ and $\left.M_{s} \sim 7\right) . V_{A} \equiv B_{0} / \sqrt{4 \pi \rho}=1 . a$ (sound speed) $=0.1$. $\delta V \sim 0.7$. Alfvén modes follow the GS95 scalings. Slow modes follow the GS95 anisotropy. But velocity spectrum of slow modes is uncertain. Fast modes are isotropic.

The super-Alfvénic turbulence simulations suggested that the picture above was true at sufficiently small scales at which Alfvén speed $V_{A}$ was larger than the turbulent velocity $v_{l}$.

Fig. 17illustrate that even in highly supersonic regime, where it was customary to claim that the modes were completely blended, the decomposition reveals a regular structure of MHD modes that corresponds to the expectation of the compressible extension of the GS95 theory.

Surely, one can debate whether the adopted technique is reliable. Indeed, the technique above is statistical in nature. That is, we separate each MHD mode with respect to the mean magnetic field $\mathbf{B}_{0}$. This procedure is affected by the wandering of large scale magnetic field lines, as well as density inhomogeneitie: 6 .

Nevertheless, CL03 demonstrated that the technique gave statistically correct results. For instance, in low $\beta$ regime, the velocity of a slow mode is nearly parallel to the local mean magnetic field. Therefore, for low $\beta$ plasmas, we can obtain velocity statistics for slow modes in real space as follows. First, the direction of the local mean magnetic field was measured using the local magnetic field. Second, the calculation of the second order structure function for slow modes was defined by the

${ }^{6}$ One way to remove the effect by the wandering of field lines is to drive turbulence anisotropically in such a way as $k_{\perp, L} \delta V \sim k_{\|, L} V_{A}$, where $k_{\perp, L}$ and $k_{\|, L}$ stand for the wavelengths of the driving scale and $\delta V$ is the r.m.s. velocity. By increasing the $k_{\perp, L} / k_{\|, L}$ ratio, we can reduce the degree of mixing of different wave modes. 

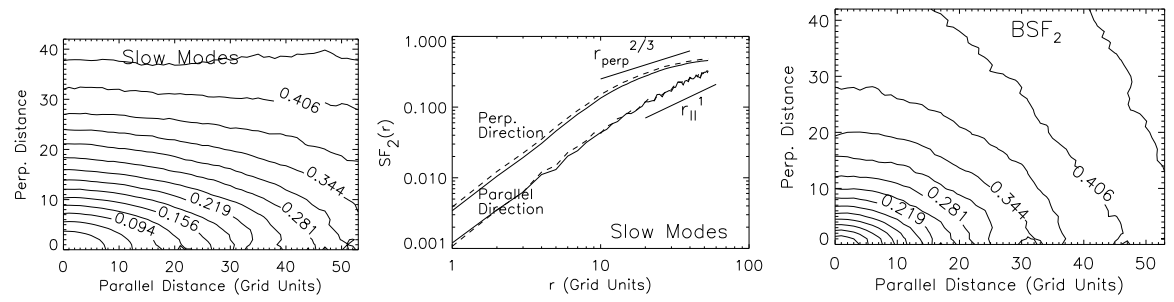

Fig. 18 Comparison between Fourier space method and real space method. (a)left: From real space calculation. $M_{s} \sim 7$. (b)middle: Solid: Fourier space. Dashed: real space. $M_{s} \sim 7$. (c)right: Similar plot for $M_{s} \sim 2.3$.

formula $\mathrm{vSF}_{2}(\mathbf{r})=<\left|(\mathbf{v}(\mathbf{x}+\mathbf{r})-\mathbf{v}(\mathbf{x})) \cdot \hat{\mathbf{B}}_{l}\right|^{2}>$, where $\hat{\mathbf{B}}_{l}$ is the unit vector along the local mean field.

Fig. 18 (a) shows the contours obtained by the method for the high sonic Mach number run. In Fig. 18 b), we compare the result obtained this way (dashed lines) and using CL03 technique. A similar plot for the mildly supersonic case is presented in Fig. 18(c).

How physical is this decomposition? If the coupling between the modes is strong in MHD turbulence one cannot talk about three different energy cascades. Indeed, the compressible MHD turbulence is a highly non-linear phenomenon and it has been thought that Alfvén, slow and fast modes are strongly coupled. Nevertheless, one may question whether this is true. A remarkable feature of the GS95 model is that Alfvén perturbations cascade to small scales over just one wave period, while the other non-linear interactions require more time. Therefore one might expect that the non-linear interactions with other types of waves should affect Alfvénic cascade only marginally. Moreover, since the Alfvén waves are incompressible, the properties of the corresponding cascade may not depend on the sonic Mach number.

The generation of compressible motions (i.e. radial components in Fourier space) from Alfvénic turbulence is a measure of mode coupling. How much energy in compressible motions is drained from Alfvénic cascade? According to closure calculations [78], the energy in compressible modes in hydrodynamic turbulence scales as $\sim M_{s}^{2}$ if $M_{s}<1$. CL03 conjectured that this relation can be extended to MHD turbulence if, instead of $M_{S}^{2}$, we use $\sim(\delta V)_{A}^{2} /\left(a^{2}+V_{A}^{2}\right)$. (Hereinafter, we define $V_{A} \equiv B_{0} / \sqrt{4 \pi \rho}$, where $B_{0}$ is the mean magnetic field strength.) However, since the Alfvén modes are anisotropic, this formula may require an additional factor. The compressible modes are generated inside the so-called GS95 cone, which takes up $\sim(\delta V)_{A} / V_{A}$ of the wave vector space. The ratio of compressible to Alfvénic energy inside this cone is the ratio given above. If the generated fast modes become isotropic (see below), the diffusion or, "isotropization" of the fast wave energy in the wave vector space increase their energy by a factor of $\sim V_{A} /(\delta V)_{A}$. This results in

$$
\frac{\delta E_{c o m p}}{\delta E_{A l f}} \approx \frac{\delta V_{A} V_{A}}{V_{A}^{2}+c_{S}^{2}}
$$


where $\delta E_{\text {comp }}$ and $\delta E_{A l f}$ are energy of compressible and Alfvén modes, respectively. Eq. (33) suggests that the drain of energy from Alfvénic cascade is marginal when the amplitudes of perturbations are weak, i.e. $(\delta V)_{A} \ll V_{A}$. Results of numerical calculations shown in CL02 support these theoretical considerations. This justifie 7 our treating modes separately.

\subsection{Other ways of decomposition into fundamental modes}

Kowal \& Lazarian (2010, [79] henceforth KL10) extended the CL03 technique by introducing an additional step before the Fourier separation, in which we decompose each component of the velocity field into orthogonal wavelets using discrete wavelet transform:

$$
\mathbf{U}\left(a, \mathbf{w}_{l m n}\right)=a^{-N / 2} \sum_{\mathbf{x}_{i j k}} \psi\left(\frac{\mathbf{x}_{i j k}-\mathbf{w}_{l m n}}{a}\right) \mathbf{u}\left(\mathbf{x}_{i j k}\right) \Delta^{N} \mathbf{x},
$$

where $\mathbf{x}_{i j k}$ and $\mathbf{w}_{l n m}$ are $N$-dimensional position and translation vectors, respectively, $a$ is the scaling parameter, $\mathbf{u}\left(\mathbf{x}_{i j k}\right)$ is the velocity vector field in the real space, $\mathbf{U}\left(\mathbf{x}_{i j k}\right)$ is the velocity vector field in the wavelet space, and $\psi$ is the orthogonal analyzing function called wavelet. The sum in the equation is taken over all position indices. KL10 use Daubechies wavelet as an analyzing function and fast discrete version of the wavelet transform, as a result they obtained a finite number of wavelet coefficients. After the wavelet transform of the velocity the Fourier representation of each wavelet coefficient was calculated and perform individual separation into the MHD modes was performed in the Fourier space using the CL03 method and then update the Fourier coefficients of all MHD waves iterating over all wavelets. In this way KL06 obtained a Fourier representation of the Alfvén, slow and fast waves. The final step is the inverse Fourier transform all all wave components.

This additional step allows for important extension of the CL03 method, namely, allows for the local definition of the mean magnetic field and density used to calculate $\alpha$ and $D$ coefficients. Since the individual wavelets are defined locally both in the real and Fourier spaces, the averaging of the mean field and density is done only within the space of each wavelet.

The study in KL10 provided results consistent with the CL03 and it extended the decomposition to new physical cases. For instance, Fig. 19 shows the anisotropy for subAlfvénic turbulence which agrees well with that obtained in CL03.

Another way to decompose into modes using structure functions has been recently proposed and tested by one of the authors (AB). In this method the separation vector $\mathbf{l}$ of the structure function plays the role of the wavenumber, because there is a correspondence relation between one-dimensional structure function along the

\footnotetext{
${ }^{7}$ A claim in the literature is that a strong coupling of incompressible and compressible motions is required to explain simulations that show fast decay of MHD turbulence. There is not true. The incompressible motions decay themselves in just one Alfvén crossing time.
} 

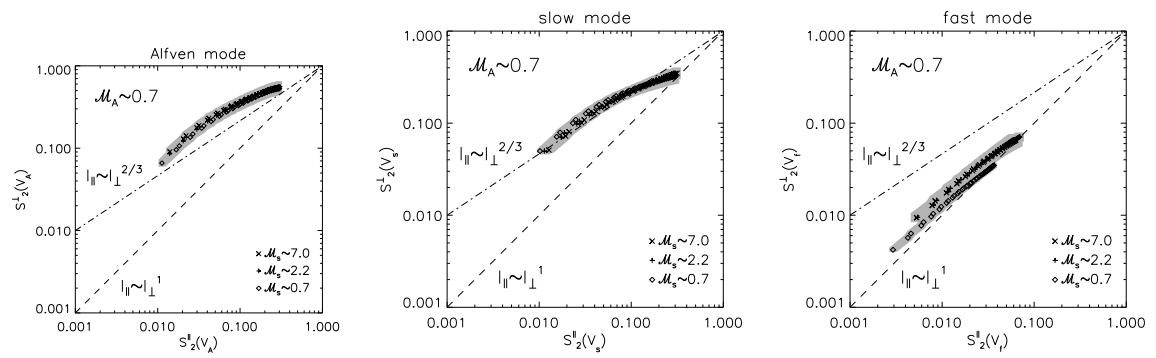

Fig. 19 Anisotropy of the Alfvén, slow and fast modes. To show the anisotropy we use the $2^{\text {nd }}$ order total structure functions, parallel and perpendicular to the local mean magnetic field. Points correspond to the mean profiles of the structure functions averaged over several snapshots. The gray areas under points correspond to the degree of departures of the structure functions in time. From KL10.

certain line and the power spectrum along the same line. Fig.20 shows the contours of the structure function corresponding to each mode obtained in datacubes from $M_{s}=10$ supersonic simulations used earlier in [41] (see also $\$$ 5.4). The anisotropies of each mode show the same behavior as in the earlier discussed global decomposition method, see Fig. 17. There are two advantages in using the new decomposition method. First, it is computationally efficient, as the structure functions can be calculated by the Monte-Carlo method which samples only a fraction of data points. This way, the very high resolution simulations can be processed in a reasonable time. The second advantage is that the structure function is a local measurement, so we can measure spectral characteristics of the modes in a highly inhomogeneous situations. This method has been applied to the decomposition of MHD turbulence obtained in high-resolution cosmological simulation of a galaxy cluster [80]. The cluster environments has been notoriously difficult to analyze due to the strong dependence of all quantities on the distance to the center. The new method was used to calculate the SFs in concentric shells around the cluster center. Among other things the aforementioned paper estimated the fraction of the fast mode to around 0.25 , which is fairly high for subsonic to trans-sonic cluster environment. We hypothesized that this is due to the way the cluster turbulence is driven - through mergers, which are essentially compressible trans-sonic motions.

\subsection{Decomposition into solenoidal and potential modes}

KL10 also used a different decomposition of the velocity field. Using the Hodge generalization of the Helmholtz theorem we can split an arbitrary vector field $\mathbf{u}$ into three components:

$$
\mathbf{u}=\mathbf{u}_{p}+\mathbf{u}_{s}+\mathbf{u}_{l},
$$

where each component has specific properties: 


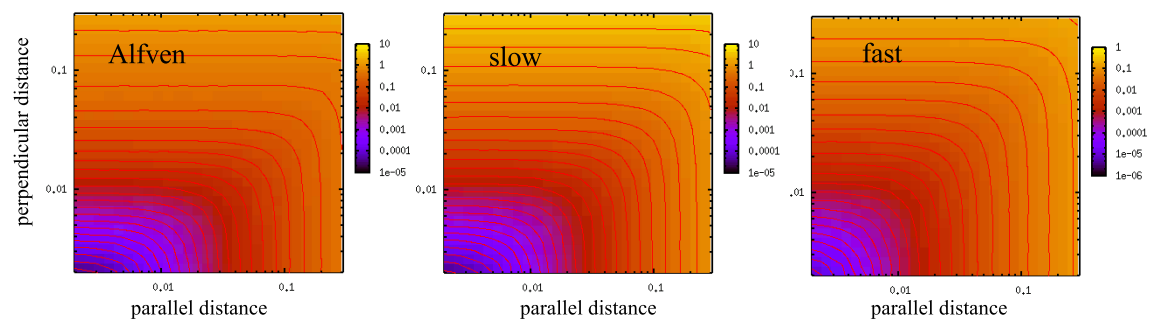

Fig. 20 Anisotropy of the Alfvén, slow and fast modes as evidenced by the contours of the second order structure function. Here we used the new SF decomposition method. The Alfvén and slow mode exhibit scale-dependent anisotropy, while the fast mode is almost isotropic.

a) Potential component ( $\left.\mathbf{u}_{p}\right)$ - it is curl-free component, i.e. $\nabla \times \mathbf{u}_{p}=0$, so it stems from a scalar potential $\phi$ :

$$
\mathbf{u}_{p}=\nabla \phi .
$$

The scalar potential $\phi$ is not unique. It is defined up to a constant. This component describes the compressible part of the velocity field.

b) Solenoidal component $\left(\mathbf{u}_{s}\right)$ - it is divergence-free component, i.e. $\nabla \cdot \mathbf{u}_{s}=0$, so it stems from a vector potential $\mathscr{A}$ :

$$
\mathbf{u}_{s}=\nabla \times \mathscr{A} .
$$

The vector potential $\mathscr{A}$ also is not unique. It is defined only up to a gradient field. In the case of velocity this component describes the incompressible part of the field.

c) Laplace component $\left(\mathbf{u}_{l}\right)$ - it is both divergence-free and curl-free. Laplace component comes from a scalar potential which satisfies the Laplace differential equation $\Delta \phi=0$.

Thus the decomposition can be rewritten in the form:

$$
\mathbf{u}=\nabla \times \mathscr{A}+\nabla \phi+\mathbf{u}_{l} .
$$

The results of this decomposition are illustrated in Fig. 21

It is clear that the compressible components of velocity correspond to shocks, while the incompressible part is dominated by GS95-type motions.

Table 5 illustrates how the percentage of energy changes within different components of the flow. It is clear from the table that even for highly compressible magnetized supersonic flows most of the energy is residing in the incompressible motions. In terms of fundamental modes the Alfvén modes dominate. However, the role of the fast modes increases with the increase of the sonic Mach number. 

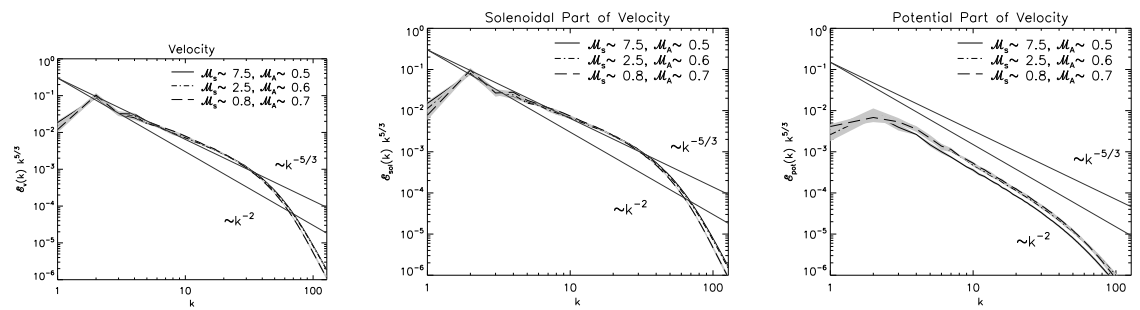

Fig. 21 Spectra of the solenoidal and potential parts of velocity for subAlfvénic turbulence. From KL10.

Table 5 Percentage amount of the kinetic energy contained within each velocity component. Errors correspond to a measure of the time variation.

\begin{tabular}{ccccccc}
\hline \hline $\mathscr{M}_{s}$ & $\mathscr{M}_{A}$ & $V_{\text {incomp. }}$ & $V_{\text {comp. }}$ & $V_{A}$ & $V_{s}$ & $V_{f}$ \\
\hline$\sim 0.7$ & $\sim 0.7$ & $96.5^{ \pm 0.8}$ & $3.3^{ \pm 0.8}$ & $58^{ \pm 4}$ & $37^{ \pm 3}$ & $4.8^{ \pm 0.7}$ \\
$\sim 2.2$ & $\sim 0.7$ & $93^{ \pm 2}$ & $7^{ \pm 2}$ & $58^{ \pm 5}$ & $33^{ \pm 4}$ & $9^{ \pm 2}$ \\
$\sim 7.0$ & $\sim 0.7$ & $92^{ \pm 2}$ & $7^{ \pm 2}$ & $56^{ \pm 4}$ & $36^{ \pm 4}$ & $8.0^{ \pm 0.7}$ \\
\hline$\sim 0.7$ & $\sim 7.4$ & $95^{ \pm 2}$ & $5^{ \pm 2}$ & $52^{ \pm 4}$ & $42^{ \pm 4}$ & $6.2^{ \pm 0.8}$ \\
$\sim 2.3$ & $\sim 7.4$ & $86^{ \pm 1}$ & $14^{ \pm 2}$ & $47^{ \pm 3}$ & $37^{ \pm 4}$ & $16^{ \pm 2}$ \\
$\sim 7.1$ & $\sim 7.1$ & $84^{ \pm 2}$ & $16^{ \pm 2}$ & $47^{ \pm 4}$ & $33^{ \pm 4}$ & $20^{ \pm 2}$ \\
\hline
\end{tabular}

\subsection{Density scalings}

The properties of density in supersonic ISM turbulence has always been of interest to astronomers due to its applications to star formation. The density is thought be be associated primarily with the slow mode, since this is the mode that perturb density the most in low-beta supersonic fluid. However, the structure function of density was generally observed to be very different from the structure function of the velocity of the slow mode. In particular, while slow mode show well-pronounced scale-dependent anisotropy, see Fig. 17, the structure function of density was almost isotropic, see Fig. 22. This mysterious difference has made applications of our knowledge of supersonic MHD turbulence to the case of star formation difficult.

However, [41] proposed a simple picture which both unraveled the mystery and further shed light on the dynamics of density in supersonic MHD. It turned out that the second-order structure function method work appropriately only if the quantity in question has a Gaussian distribution. If we use it on density, which distributed approximately log-normally and has high-density tail, this greatly favor high-density regions or clumps. The apparent isotropy, therefore, is an artifact of these clumps being distributed randomly in space. Furthermore, the flat spectrum of density comes from the same effect, namely, high-density clumps act as a delta-functions and produce flat spectrum. When we use log-density instead of density, the spectra become steeper and the second-order structure function shows remarkable scale-dependent anisotropy, see Fig. 22 . 

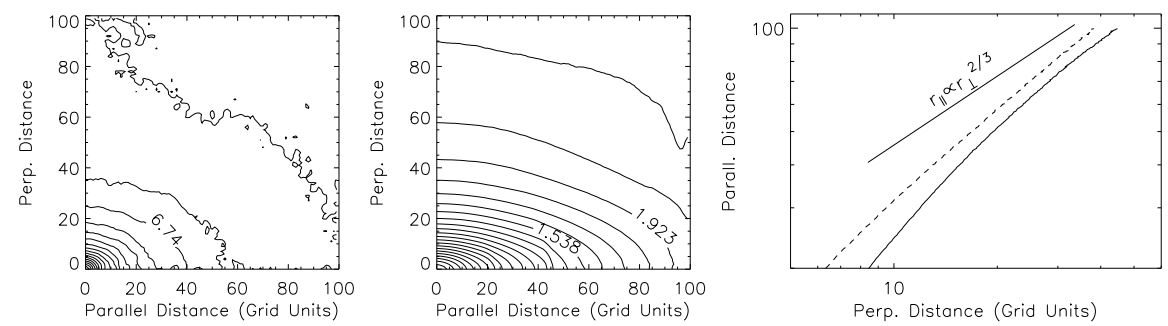

Fig. 22 Contours of the structure function of density (left), log-density (center) and the anisotropy of log-density (right). From 441

So it turned out that while the perturbations of density and its log-normal PDF are created by random slow shocks, the structure of density has an imprint from Alfvénic driving, the same imprint the structure of slow mode velocity has.

\subsection{Viscosity-dominated regime of MHD turbulence}

In this section, we focus on the effects of viscosity. In strong hydrodynamic turbulence energy is injected at a scale $L$, and cascades down to smaller scales without significant viscous losses until it reaches the viscous damping scale $l_{d v}$. The Kolmogorov energy spectrum applies to the inertial range, i.e. all scales between $L$ and $l_{d v}$. This simple picture becomes more complicated when we deal with MHD turbulence because there are two dissipation scales - the velocity damping scale $l_{d v}$ and the magnetic diffusion scale $l_{d m}$, where magnetic structures are dissipated. In fully ionized collisionless plasmas (e.g. the hottest phases of the ISM), $l_{d v}$ is less than an order of magnitude larger than $l_{d m}$, but both scales are very small. However, in partially ionized plasmas (e.g. the warm or cold neutral phase of the ISM), the two dissipation scales are very different and $l_{d v} \gg l_{d m}$. In the Cold Neutral Medium (see [81] for a list of the ISM phases) neutral particle transport leads to viscous damping on a scale which is a fraction of a parsec. In contrast, in these same phases $l_{d m} \sim 100 \mathrm{~km}$.

This has a dramatic effect on the energy cascade model in a partially ionized medium. When the energy reaches the viscous damping scale $l_{d v}$, kinetic energy will dissipate there, but the magnetic energy will not. In the presence of dynamically important magnetic field, Cho, Lazarian, \& Vishniac ([82]; hereafter CLV02b) reported a completely new regime of turbulence below the scale at which viscosity damps kinetic motions of fluids. They showed that magnetic fluctuations extend below the viscous damping scale and form a shallow spectrum $E_{b}(k) \sim k^{-1}$. This spectrum is similar to that of the viscous-convective range of a passive scalar in hydrodynamic turbulence. 

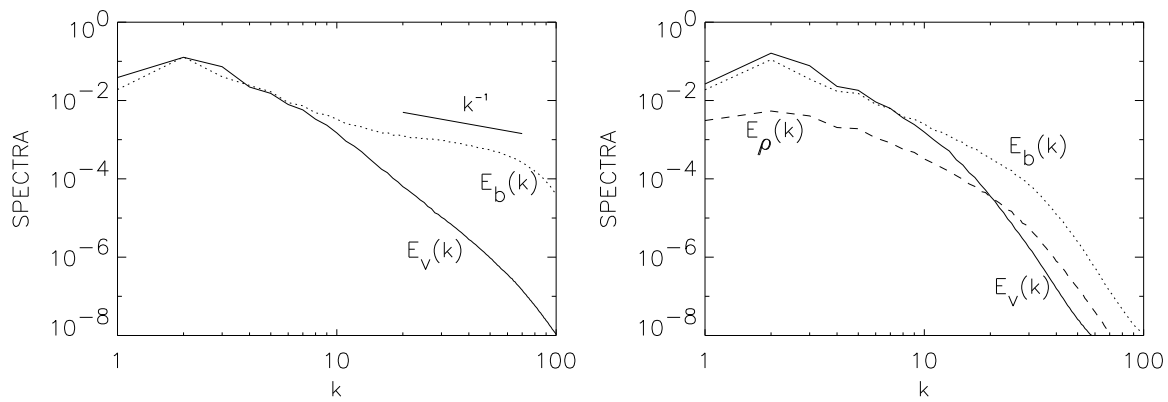

Fig. 23 Viscous damped regime (viscosity $>$ magnetic diffusivity). Due to large viscosity, velocity damps after $k \sim 10$. (a) Left: Incompressible case with $384^{3}$ grid points. Magnetic spectra show a shallower slope $\left(E_{b}(k) \propto k^{-1}\right)$ below the velocity damping scale. We achieve a very small magnetic diffusivity through the use hyper-diffusion. From CLV02b. (b) Right: Compressible case with $216^{3}$ grid points. Magnetic and density spectra show structures below the velocity damping scale at $k \sim 10$. The structures are less obvious than the incompressible case because it is relatively hard to achieve very small magnetic diffusivity in the compressible run. From CL03.

A further numerical study of the viscosity-damped MHD turbulence was presented in CL03 and [83]. Figure23] compares the results for this regime obtained for compressible and incompressible MHD turbulence.

Fig. 24]show structures and spectra in supersonic viscous MHD simulations, emulating conditions in the molecular clouds, where high ambipolar diffusion could result in drag and damping of kinetic motions. Remarkably, the kinetic and magnetic spectra are very similar to the incompressible and weakly compressible cases. However, the structures, observed in the datacubes are completely different. The supersonic structures are completely dominated by the current sheets, which are also density sheets. This is because currents sheets has low magnetic pressure and this has to be compensated by gas pressure.

The theoretical study of weakly compressible viscously damped case was performed in Lazarian, Vishniac \& Cho (2004, [84] henceforth LVC04). Below we present a brief summary of the theory. Following the usual treatment of ordinary strong MHD turbulence, we define the wavenumbers $k_{\|}$and $k_{\perp}$ as the components of the wavevector measured along the local mean magnetic field and perpendicular to it, respectively. Here the local mean magnetic field is the direction of the locally averaged magnetic field, which depends not only on the location but also the volume over which the average is taken. See [85, 48] for details.

Lazarian, Vishniac, \& Cho ([84], henceforth LVC04) proposed a theoretical model for viscosity-damped MHD turbulence. We summarize the model as follows.

Since there is no significant velocity fluctuation below $l_{d v}$, the time scale for the energy cascade below $l_{d v}$ is fixed at the viscous damping scale. Consequently the energy cascade time scale $t_{c a s}$ is scale-independent below $l_{d v}$ and the requirement for a scale independent energy transfer rate $b_{l}^{2} / t_{\text {cas }}$ yields

$$
b_{l} \sim \text { constant, or } E_{b}(k) \sim k^{-1},
$$



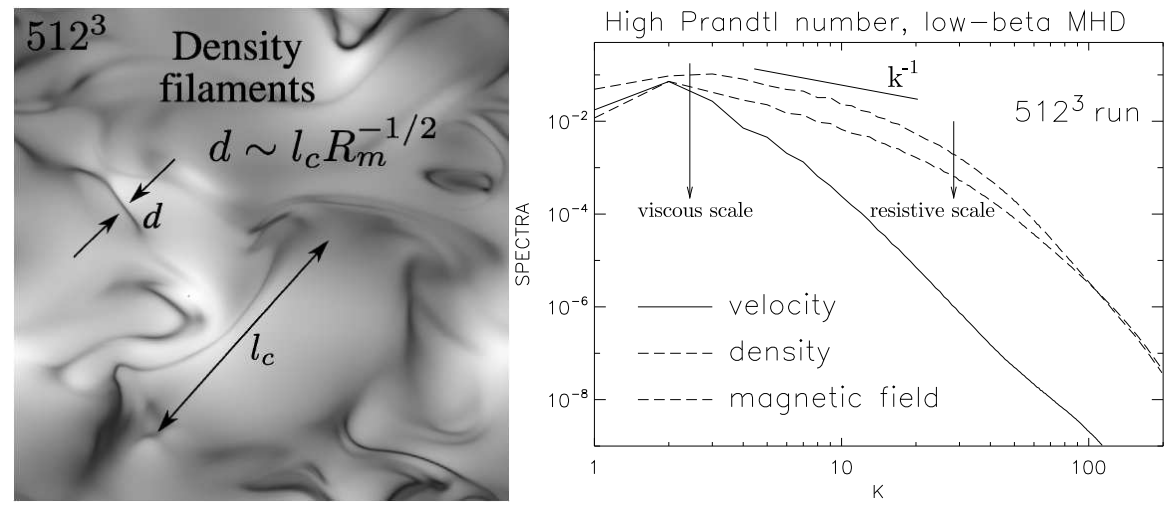

Fig. 24 Simulations of supersonic, viscously damped MHD turbulence, with high viscosity emulating high drag from ambipolar diffusion in molecular clouds. Left: Filaments of density created by magnetic compression of the gas in this regime. Darker regions correspond to higher density. The viscous damping scale $l_{c}$ is much larger than the current sheet thickness $d$. This creates large observed density contrasts. Right: The spectra of density, velocity and magnetic field in this case. While the density and magnetic spectra are similar, the velocity spectrum has a cutoff due to high viscosity. Note that the resistive scale in this regime is not $L / R m$ but $L R m^{-1 / 2}$.

where $k E_{b}(k) \sim b_{l}^{2}$.

In LVC04, we assume that the curvature of the magnetic field lines changes slowly, if at all, in the cascade:

$$
k_{\|} \sim \text { constant. }
$$

This is consistent with a picture in which the cascade is driven by repeated shearing at the same large scale. It is also consistent with the numerical work described in CLV02b, which yielded a constant $k_{\|}$throughout the viscously damped nonlinear cascade. A corollary is that the wavevector component in the direction of the perturbed field is also approximately constant, so that the increase in $k$ is entirely in the third direction.

The kinetic spectrum depends on the scaling of intermittency. In LVC04, we define a filling factor $\phi_{l}$, which is the fraction of the volume containing strong magnetic field perturbations with a scale $l \sim k^{-1}$. We denote the velocity and perturbed magnetic field inside these sub-volumes with a "“" so that

$$
v_{l}^{2}=\phi_{l} \hat{v}_{l}^{2},
$$

and

$$
b_{l}^{2}=\phi_{l} \hat{b}_{l}^{2}
$$

We can balance viscous and magnetic tension forces to find

$$
\frac{v}{l^{2}} \hat{v}_{l} \sim \max \left[\hat{b}_{l} k_{c}, B_{0} k_{\|, c}\right] \hat{b}_{l} \sim k_{c} \hat{b}_{l}^{2},
$$


where $k_{c} \sim 1 / l_{d v}$ and $k_{\|, c}$ is the parallel component of the wave vector corresponding to the perpendicular component $k_{c}$. We used the GS95 scaling $\left(B_{0} k_{\|, c} \sim b_{l} k_{c}\right)$ and $\hat{b}_{l} \geq b_{l}$ to evaluate the two terms in the square braces. Motions on scales smaller than $l_{d v}$ will be continuously sheared at a rate $\tau_{s}^{-1}$. These structures will reach a dynamic equilibrium if they generate a comparable shear, that is

$$
\frac{\hat{v}_{l}}{l} \sim \tau_{s}^{-1} \sim \text { constant. }
$$

Combining this with equation (43), we get

$$
\phi_{l} \sim k_{c} l
$$

and

$$
E_{v}(k) \sim k^{-4} .
$$

Note that equation (43) implies that kinetic spectrum would be $E_{v}(k) \sim k^{-5}$ if $\phi_{l}=$ constant.

\subsection{Application of results to collisionless fluids}

Some astrophysical magnetized fluids are collisionless, meaning the typical collision frequency is lower than the gyrofrequency. It is important to understand to what extend the results obtained for MHD can also be applied to such environments. The effective collisionality of the medium depends on the collective effects of magnetic scattering of ions. For instance, gyroresonance instability induced by large scale compressions produces small scale perturbations that induce efficient scattering of charged particles [86, 87, 88]. Thus the free energy of turbulent environment makes plasmas, effectively, much more collisional. Another example of this is a collisionless shock which excite plasma waves and lead to effective particle thermalization.

Furthermore, some subsets of MHD equations, such as reduced or Alfvénic MHD, which we studied in great detail in Sections 3 and 4, are actually applicable to fully collisionless plasmas, because Alfvénic motions are essentially $[\mathbf{E} \times \mathbf{B}]$ drift motions, rely only on magnetic tension and do not require collisions, see, e.g., [55].

A recent study in [89], using a closure for anisotropic plasma pressure, showed that for a reasonable choice of the relaxation term the collisionless fluids behave similar to MHD. Thus we expect that both MHD turbulence scaling relations and the results of turbulent dynamo that we discussed in above are applicable to collisionless turbulent astrophysical plasmas above the effective collisional scale. The measurements in the solar wind indicate that the effective MHD scales could be as low as the ion skip depth or the ion Larmor radius. 

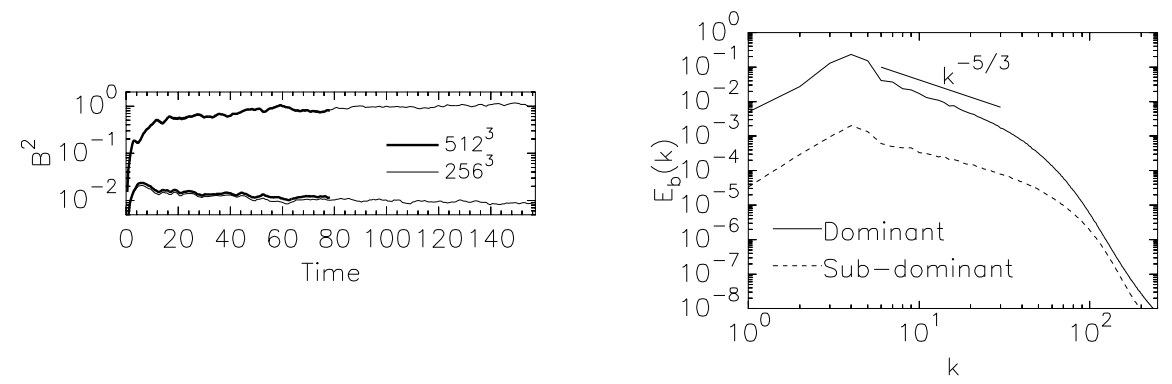

Fig. 25 Left panel: The time dependence of energy densities for the dominant and subdominant waves. Right panel: Energy spectra of the dominant and subdominant waves. The spectrum of the subdominant flux is shallower that for the dominant wave. From CL14.

\subsection{Outlook on relativistic turbulence}

When electromagnetic energy density is much larger than the rest mass energy density of matter, the electromagnetic fields becomes essentially force-free, which is described with the so-called relativistic force-free approximation. The examples of such environments include electron-positron pulsar magnetospheres and the inner parsec-scale AGN jets.

Numerical simulations of force-free MHD turbulence by [90] reported anisotropic Goldreich-Sridhar scalings, similar to the ones observed in Alfvénic turbulence, which was earlier conjectured by [91]. More recently a challenging numerical work of studying imbalanced relativistic turbulence was performed in Cho \& Lazarian (2014, [92], henceforth CL14). Fig.25] shows the energy densities and energy spectra of the dominant and subdominant components.

The results of this study agree with the predictions of the Beresnyak-Lazarian [44] model for non-relativistic imbalanced turbulence that we discussed in Section 4. In fact, CL14 concluded that the magnetic spectrum of dominant waves is steeper than that of sub-dominant waves and the dominant waves exhibit anisotropy which is weaker than predicted in [30] while the sub-dominant waves exhibit stronger than GS95 anisotropy. In addition, CL14 showed that The energy density ratio of the dominant to subdominant waves scales in proportion to the ratio of the energy injection rates to the power of $n$, i.e. $\left(\varepsilon_{+} / \varepsilon-\right)^{n}$, where $n>2$, which is also consistent with the Beresnyak-Lazarian [44] predictions.

The work on imbalanced and balanced relativistic turbulence strongly indicate that the nature of turbulence does not significantly change with the transfer to the relativistic regime. This conclusion is suggestive that the models based on the Goldreich-Sridhar turbulence, e.g. the turbulent reconnection model [45] can be extended to relativistic phenomena (see discussion in [93]). 


\section{Intermittency of MHD turbulence}

\subsection{General considerations}

So far our focus in the review was on the turbulence self-similarity. This property, which is also called scale-invariance, implies that fluid turbulence can be reproduced by the magnification of some part of it.

At the dissipation scales the self-similarity is known to fail with turbulence forming non-Gaussian dissipation structures as exemplified, e.g. in [59]. Interestingly enough, present-day research shows that self-similarity is not exactly true even along the inertial range. Instead the fluctuations tend to get increasingly sparse in time and space at smaller scales. This property is called intermittency. Note, that the power-law scaling does not guarantee the scale-invariance or absence of intermittency.

One way to do such studies is to investigate the scaling powers of longitudinal velocity fluctuations, i.e. $(\delta V)^{p}$, where $\delta V \equiv(\mathbf{V}(\mathbf{x}+\mathbf{r})-\mathbf{V}(\mathbf{x})) \mathbf{r} / r$. The infinite set of various powers of $S^{p} \equiv\left\langle(\delta V)^{p}\right\rangle$, where $\langle.$.$\rangle denote ensemble 8$ averaging, is equivalent to the p.d.f. of the velocity increments. For those powers one can write $S^{p}(r)=a_{p} r_{p}^{\xi}$ to fully characterize the isotropic turbulent field in the inertial range. While the scaling coefficients $a_{p}$ are given by the values of the function $S^{p}$ e.g. at the injection scale, the scaling exponents $\xi_{p}$ are very non-trivial. It is possible to show that for a self-similar flow the scaling exponents are linear function of $n$, i.e. $\xi_{p} \sim p$, which for Kolmogorov model $S^{1} \sim v_{l} \sim l^{1 / 3}$ gives $\xi_{p}=p / 3$. Experimental studies, however, give different results which shows that the Kolmogorov model is an oversimplified one.

MHD turbulence, unlike hydro turbulence, deals not only with velocity fluctuations, but also with the magnetic ones. The intermittencies of the two fields can be different. In addition, MHD turbulence is anisotropic as magnetic field affects motions parallel to the local direction of $\mathbf{B}$ very different. This all makes it more challenging to understand the properties of MHD intermittency more interesting.

An interesting and yet not understood property of structure functions, however, helps to extend the range over which $S^{p}$ can be studied. Benzi et al [94] reported that for hydrodynamic turbulence the functions $S^{p}\left(S^{3}\right)$ exhibit much broader power-law range compared to $S^{p}(r)$. While for the inertial range a similarity in scaling of the two functions stem from the Kolmogorov scaling $S^{3} \sim r$, the power-law scaling of $S^{p}\left(S^{3}\right)$ protrudes well beyond the inertial range into the dissipation range 9 . This observation shows that the dissipation "spoils" different orders of $S$ in the same manner. Therefore there is no particular need to use the third moment, but one can use any other moment $S^{m} \sim r^{m}$ and obtain a good power law of the function $S^{p} \sim$ $\left(S^{m}\right)^{\xi_{p} / \xi_{m}}$ (see [59]).

\footnotetext{
${ }^{8}$ In astrophysics spatial or temporal averaging is used.

${ }^{9}$ In practical terms this means that instead of obtaining $S^{p}$ as a function of $r$, one gets $S^{p}$ as a function of $S^{3}$, which is nonlinear in a way to correct for the distortions of $S^{p}$.
} 

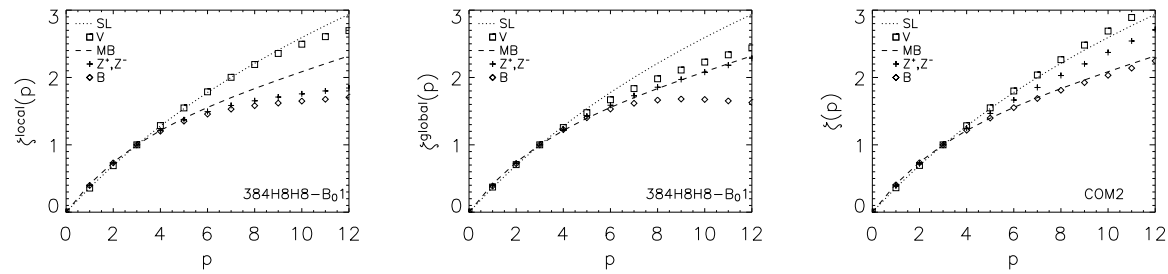

Fig. 26 left panel: Intermittency exponents for incompressible MHD turbulence in perpendicular directions in the local frame. The velocity exponents show a scaling similar to the She-Leveque model. The magnetic field shows a different scaling. central panel: Intermittency exponents for incompressible MHD turbulence in the global frame. Note that the result for $z^{ \pm}$is very similar to the Müller-Biskamp model [97]. right panel: Intermittency exponents for superAlfvénic compressible turbulence in the global frame. From CLV03

\subsection{She-Leveque model of intermittency}

A successful model to reproduce both experimental hydro data and numerical simulations is She-Leveque (1994, [95]) model. According to [96] this model can be derived assuming that the energy from large scale is being transferred to $f<1$ less intensive eddies and $1-f$ of more intensive ones. The scaling relations suggested in [95] related $\zeta_{p}$ to the scaling of the velocity $V_{l} \sim l^{1 / g}$, the energy cascade rate $t_{l}^{-1} \sim l^{-x}$, and the co-dimension of the dissipative structures $C$ :

$$
\zeta_{p}=\frac{p}{g}(1-x)+C\left(1-(1-x / C)^{p / g}\right) .
$$

For incompressible turbulence these parameters are $g=3, x=2 / 3$, and $C=2$, implying that dissipation happens over 1D structures (e.g. vortices). So far the SheLeveque scaling has done well in reproducing the intermittency of incompressible hydrodynamic turbulence.

\subsection{Intermittency of incompressible turbulence}

In their pioneering study [97] applied the She-Leveque model to incompressible MHD turbulence and attracted the attention of the MHD researchers to this tool. They used Elsässer variables and claimed that their results are consistent with dissipation within 2D structures (e.g. 2D current sheets). The consequent study [48] used velocities instead of Elsässer variables and provided a different answer, namely, that the dimension of dissipation structures is the same as in incompressible hydro, i.e. the dissipation structures are 1D. The difference between the two results was explained in Cho, Lazarian \& Vishniac (2003, [83] henceforth CLV03). They noted that, first of all, the measurements in [97] were done in the reference frame related to the mean magnetic field, while the measurements in [48] were done in the frame 

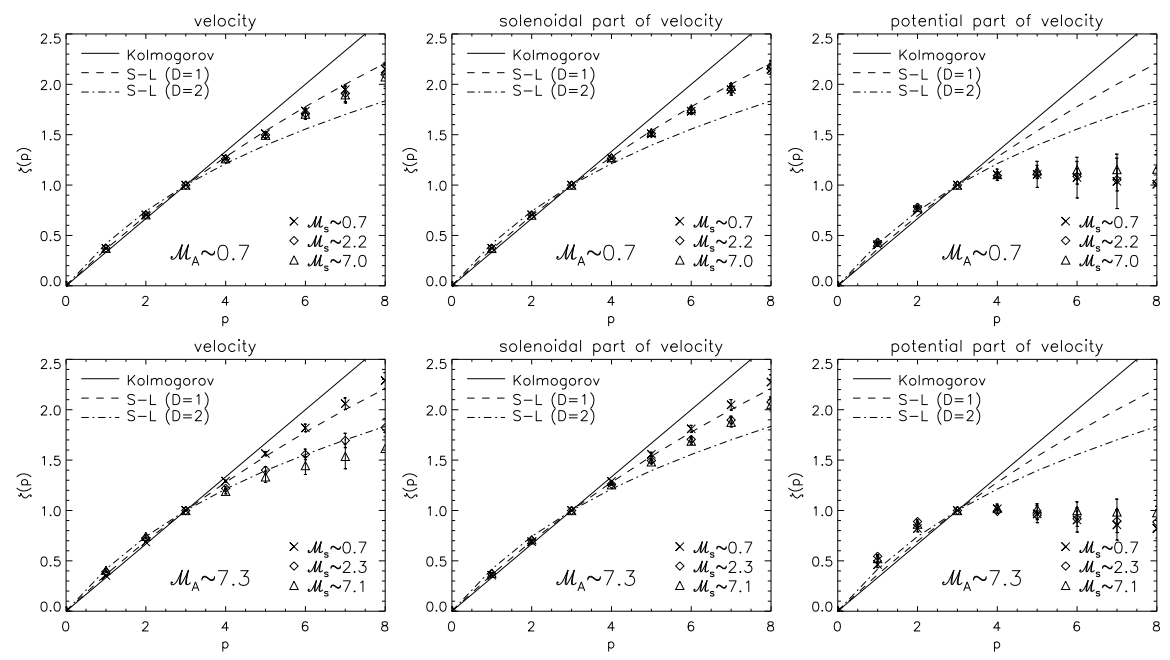

Fig. 27 Scaling exponents of the velocity (left column) and its incompressible and compressible parts (middle and right columns, respectively) for experiments with different sonic Mach numbers in two regimes: subAlfvénic (upper row) and superAlfvénic (lower row). From KL10.

related to the local magnetic field. We believe that the latter is more physically motivated frame, as it is the local magnetic field is the field that is felt by the eddies. It is also in this reference frame that the scale-dependent anisotropy predicted in the GS95 model is seen. Computations in CLV03 confirmed that the dissipation structures that can be identified as velocity vortices in the local magnetic field reference frame can also be identified with two dimensional sheets in terms of Elsässer variables in the mean magnetic field reference frame. This, first of all, confirms a mental picture where motions perpendicular to magnetic field lines are similar to hydrodynamic eddies. More importantly, it sends a warning message about the naive interpretation of the She-Leveque scalings in the MHD turbulence.

\subsection{Intermittency of compressible turbulence}

Intermittency in compressible MHD turbulence was discussed in Boldyrev (2002 [98]) who assumed that the dissipation there happens in shock:10 and therefore the dimension of the dissipation structures is 2 . The idea of the dominance of shock dis-

\footnotetext{
10 The cited paper introduces the model of compressible turbulence which it calls KolmogorovBurgers model. Within this model turbulence goes first along the Kolmogorov scaling and then, at small scales forms shocks. The model was motivated by the numerical measurements of the turbulence spectrum that indicated the index of supersonic turbulence close to $-5 / 3$. This however was shown to be an artifact of numerical simulations with lower resolution. Simulations in [99] showed that the slope with $-5 / 3$ is the result of the numerical bottleneck and the actual slope of the highly compressible turbulence is -2 , as was expected earlier.
} 
sipation does not agree well with the numerical simulations in CL02, CL03, where the dominance of the vortical motions in subAlfvénic turbulence (i.e. magnetic pressure is larger than the gaseous one) was reported. Nevertheless, numerical simulations in [100] showed that for superAlfvénic turbulence (i.e. magnetic pressure is less than the gas pressure) the dimension of the dissipation structures was gradually changing from one to somewhat higher than two as the Mach number was increasing from 0.4 to 9.5. The very fact that the superAlfvénic turbulence, which for most of the inertial scale resolvable by simulations does not have a dynamically important magnetic field is different from subAlfvénic is not surprising. The difference between the results in [100] at low Mach number and the incompressible runs in [97] deserves a discussion, however. First of all, the results in [100] are obtained for the velocity, while the results in [97] are obtained for the Elsässer. CLV03 has shown that the magnetic field and velocity have different intermittencies. Indeed, it is clear from Fig. 1 that $\zeta^{\text {magnetic }}<\zeta^{\text {velocity }}$ which means that magnetic field is more intermittent than velocity. An interesting feature of superAlfvénic simulations in Fig. 26 is that the velocity follows the She-Leveque hydro scaling with vortical dissipation, while magnetic field exhibits a pronounced dissipation in current sheets. Both features are expected if magnetic field is not dynamically important and the turbulence stays essentially hydrodynamic. We also see that the dynamically important magnetic field does changes the intermittency. The flattening of magnetic field scaling is pronounced in Fig. 26 .

A more recent study of intermittency of the velocity field of compressible turbulence was performed in KL10. In Figure 27 we show scaling exponents for the velocity and all its parts and waves calculated in the global reference frame. In the top left plot of Figure 27 we see that for the subAlfvénic turbulence the scaling exponents of velocity follow the She-Lévêque scaling with $D=1$. Supported by the theoretical considerations we can say that most of the dissipative structures are onedimensional. Even though the scalings are not perfectly independent of the value of $\mathscr{M}_{s}$, since we see somewhat lower values of $\zeta$ for higher $p$, the differences between these values for models with different sonic Mach numbers are within their error bars, thus it is relatively difficult to state that the scalings are completely independent or only weakly dependent of the values of $\mathscr{M}_{s}$. Looking in the corresponding plot for models with a weak magnetic field we clearly see that the spread of curves for different sonic Mach numbers is much higher than in the previous case. For subsonic model the scaling exponents of velocity follow very well the theoretical curve defined by the S-L scaling with parameter $D$ corresponding to one-dimensional structures. The model with $\mathscr{M}_{s} \sim 2.3$, however, follows perfectly the S-L scaling with $D=2$ corresponding to the two-dimensional dissipative structures. Moreover, models with even higher values of the sonic Mach number have the scaling exponents for $p>3$ somewhat below the S-L scaling with $D=2$. These observations suggest that the scaling exponents of the velocity change with the sonic Mach number but only in the case of weak magnetic field turbulence. The presence of a strong magnetic field significantly reduces these changes and preserves the generation of the dissipative structures of higher than one dimensions. 

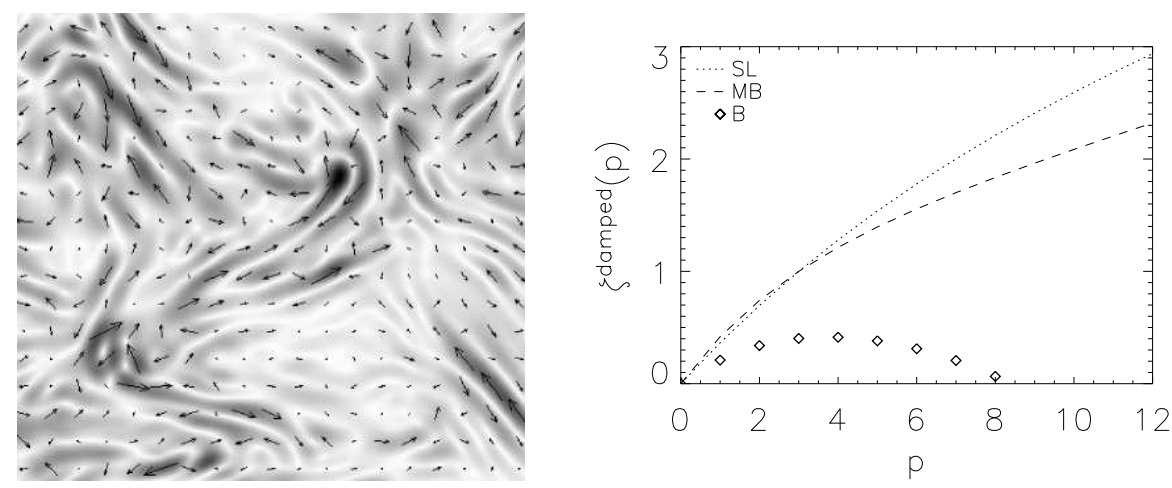

Fig. 28 The incompressible viscously damped simulations: Left: Magnetic reversals (in the plane $\perp$ to mean $\langle\mathbf{B}\rangle$ ) that create current layers and makes turbulence highly intermittent. Darker regions correspond to higher magnetic field. Right: Intermittency indexes.

After the decomposition of velocity into its incompressible and compressible parts we also calculate their scaling exponents. In the middle and right columns of Figure 27 we show the incompressible and compressible parts of the velocity field, respectively. The incompressible part it strong. It constitutes most of the velocity field thus it is not surprising that its scaling exponents are very similar to those observed in velocity. This is true in the case of subAlfvénic models, because all curves in the middle left plot in Figure 27 are tightly covering the S-L scaling with $D=1$. The similarity between the velocity and its solenoidal part is also confirmed in the case of superAlfvénic models but only for subsonic case, when the role of shocks is strongly diminished. Two supersonic models show exponents following a scaling more closer to the S-L one with $D=1$, yet still with lower values for $p>3$.

\subsection{Intermittency of viscosity-damped turbulence}

For the extreme intermittency of the magnetic field suggested in LVC04 the higher moments of structure functions $S_{p} \sim{\hat{b_{l}}}^{p} \phi_{l}$ which means that $S_{p} \sim l^{1-p / 2}$. The concentration of magnetic field in thin filaments gives rise to resistive loses that should eventually make $\xi_{p}=0$ for sufficiently large $p$. In Fig. 28 we see this general tendency for high $p$. For the absence of the more precise correspondence we may blame (a) our crude model for estimating $\xi$, (b) numerical effects, and (c) LVC04 model itself. Addressing the issue (b), we would say that the compelling arguments in the model provide $k^{-1}$ spectrum and this would provide $\xi(2)=0$ in accordance with the intermittency model above. However, due to numerical effects identified in LVC04 the spectrum of magnetic fluctuations is slightly steeper. 

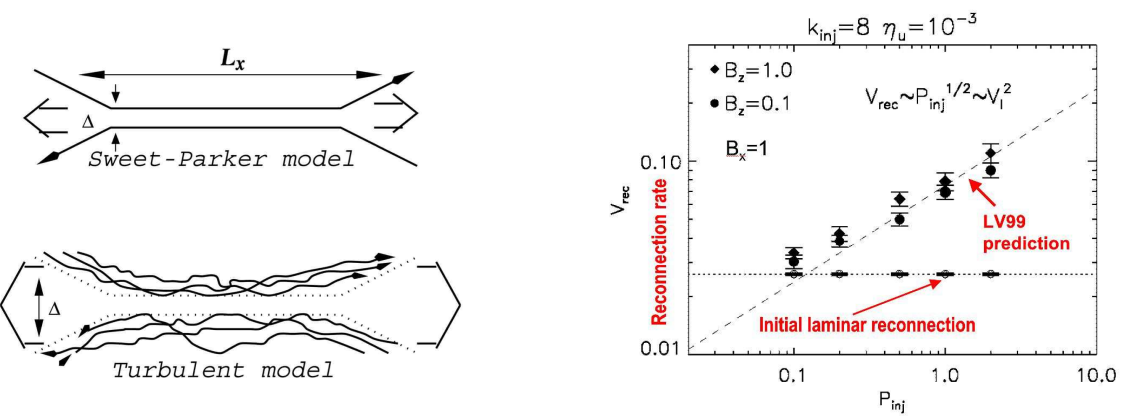

Fig. 29 Left: Upper panel. Sweet-Parker reconnection. $\Delta$ is limited by resistivity and is small. Lower panel: reconnection according to the LV99 model. $\Delta$ is determined by turbulent field wandering and can be large. From [84]. Right: Testing of LV99 model with numerical simulations in [109]. The dependence on the power of turbulent driving is shown.

\section{Selected implications of MHD turbulence and turbulent dynamo}

Astrophysical fluids are turbulent and therefore one must take into account properties of turbulence while describing astrophysical processes. We have discussed various implications in recent reviews, e.g. in [101]. Below is provided a brief summary of selected applications of the scalings obtained.

\subsection{Magnetic reconnection in the presence of MHD turbulence}

Magnetic reconnection is a long standing problem. Is it associated with the fundamental ability of magnetic flux tubes to change their topology, while being submerged within conducting fluids [102, 103]. Lazarian and Vishniac ([45] henceforth LV99) considered turbulence as the agent that makes magnetic reconnection fast (see Fig. 29p. The scheme proposed in LV99 there differs appreciably from the earlier attempts to enhance reconnection via turbulence, [104, 105, 106, 107], see [108] for a detailed comparison. The LV99 model relies on opening of reconnection region via magnetic field wondering, and for the Alfvénic turbulence model that was discussed in this review provide the scaling that are of reconnection velocity $V_{\text {rec }}$ being proportional to square root of the injection power $P_{i n j}$. Fig. 29 shows a good relation of the numerical testing in [109, 110] and the predictions. See also Lazarian et al, this volume, for a review of turbulent reconnection.

A study in Eyink, Lazarian \& Vishniac (2011, [108] henceforth ELV11) reveals a very deep relation between MHD turbulence and magnetic reconnection. In fact, it was shown back in LV99 that the predicted reconnection rates are necessary to make the GS95 model self-consistent, i.e. to resolve magnetic knots that emerge as eddy-like motions perpendicular to the direction of local magnetic field twist 
magnetic field lines. ELV11 demonstrates that the Lagrangian properties of MHD turbulence require the violation 11 of magnetic flux being frozen in and re-derives the predictions of LV99 model from this established properties. As magnetic flux frozenness is a corner stone of major astrophysical theories its violation in turbulent fluids has deep consequences. For instance, the change of our understanding of diffusion out of star forming clouds in the presence of turbulence was recently discussed in [114, 115]. The numerical confirmation of the violation of magnetic field flux freezing in turbulent fluids was reported in [116].

\subsection{Turbulence and particle acceleration}

MHD turbulence plays an important role in accelerating energetic particles. First of all, the second order Fermi acceleration can arise directly from the scattering of particles by turbulence, see, e.g., [117]. Properties of MHD turbulence that we discussed above are essential to understanding this process. If turbulence is injected at large scales, the anisotropy of Alfvénic modes at small scales makes them inefficient for scattering and acceleration of cosmic rays [118, 68]. In this situation, fast modes were identified in [68] as the major scattering and acceleration agent for cosmic rays and energetic particles in interstellar medium (see also [119, 120]). This conclusion was extended for solar environments in Petrosian, Yan \& Lazarian (2006) and intracluster medium in [121].

Turbulent magnetic field in the pre-shock and post-shock environment are important for the first order Fermi acceleration associated with shocks [122]. In particular, magnetic field enhancement compared to its typical interstellar values is important in the pre-shock region for the acceleration of high energy particles. Turbulent dynamo that we discussed in Section 2 can provide a way of generating magnetic field in the precursor of the shock. In [28] it was shown that the interactions of the density inhomogeneities pre-existing in the interstellar medium with the precursor generate strong magnetic fields in the shock precursor, which allows particle acceleration up to the energy of $10^{16} \mathrm{eV}$.

In addition, fast magnetic reconnection of turbulent magnetic field can itself induce the first order Fermi acceleration [123, 112]. Recent numerical simulations in [124], demonstrate the efficiency of this process.

\subsection{Thin structures in the Interstellar Medium}

The viscosity-dominated regime of turbulence can be responsible for the formation of structures in interstellar medium and other astrophysical environments. The mag-

\footnotetext{
11 The violation of frozen in condition in turbulence is implicit in LV99. It was stated explicitly in [111] and discussed in terms of star formation in [112]. The first formal quantitative study was performed in [113.
} 
netic pressure compresses the gas as demonstrated in Fig. 24 More importantly, extended current sheets that naturally emerge as magnetic field fluctuates in the plane perpendicular to the mean magnetic field. It was speculated in [125] that these current sheets can account for the origin of the small ionized and neutral structures (SINS) on AU spatial scales [126, 127].

Goldreich and Sridhar [128] appealed to the generation of the magnetic field in the high $P t$ turbulent plasma [129] to account for the high amplitude, but small scale fluctuations of plasma density observed in the direction of the Galactic center. They argued that the plasma viscosity parallel to magnetic field can act in the same way as the normal viscosity of unmagnetized fluids. [130] argued that the regime of dynamo in [129] and the turbulence in [84] have similarities in terms of the density enhancement that are created. Although in the case of magnetic turbulence with sufficiently strong mean magnetic field, global reversals, that [128] appeal to in compressing plasma, do not happen, the reversals of the magnetic field direction occur in the direction perpendicular to the mean magnetic field. As the mean magnetic field goes to zero, the two regimes get indistinguishable as far as the density enhancements are concerned. Thus high intensity fluctuations of plasma density towards the Galactic center may also be the result of viscosity-damped turbulence.

\subsection{Intermittent turbulent heating of interstellar gas}

E. Falgarone and her collaborators [131, 132, 133, 134] attracted the attention of the interstellar community to the potential important implications of intermittency, see also Falgarone et al, this volume, for a review. A small and transient volume with high temperatures or violent turbulence can have significant effects on the net rates of processes within the ISM. For instance, many interstellar chemical reactions (e.g., the strongly endothermic formation of $\mathrm{CH}^{+}$) might take place within very intense intermittent vortices.
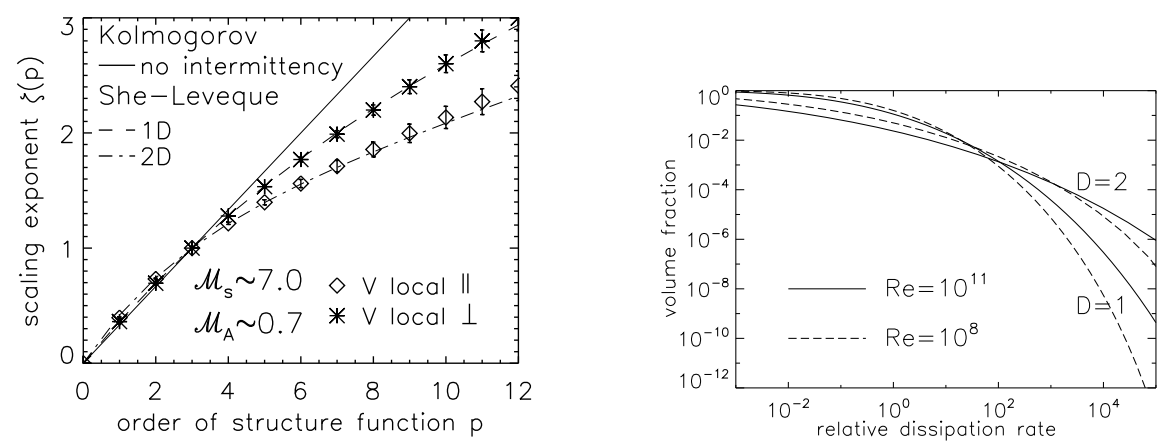

Fig. 30 Left Panel: The intermittencies of velocities in the subAlfvénic, $M_{A}=0.7$ supersonic $M_{s}=7$ MHD simulations. From [135]. Right Panel: Volume fraction with the dissipation rate is higher than the mean rate for the She-Leveque model of intermittency with $D=1$ and 2. 
The bottom part of Fig. 30 shows our calculations for the volume fractions of various dissipation rates (i.e., heating rates), based on the distribution function suggested by She, Leveque and Dubrulle. While the temperatures achieved will depend upon the cooling functions, some important conclusions are available from the analysis of Fig. 30 This figure demonstrates that even for Re $\sim 10^{11}$ the bulk of the energy dissipates within volume with dissipation rate around a factor of a few of the mean value, provided that the She-Leveque model is valid. Furthermore, only $0.1 \%$ of the volume has the dissipation rate higher than the factor of 100 of the mean value. This provides stringent constraints on what chemistry we could expect to be induced by intermittent turbulent heating.

Interestingly enough, the case of intermittency studies supports our point of the futility of the "brute force" numerical approach. For instance, for a typical ISM injection scale of $50 \mathrm{pc}$, the Reynolds number can be as high as $\operatorname{Re}=10^{11}$. In comparison, numerical simulations can only reach $\mathrm{Re} \leq 10^{5}$ for the present record resolution of $4096^{3}$.

\subsection{Suppression of instabilities by Alfvenic turbulence}

Alfvenic turbulence can suppress instabilities, in particular, streaming instability that arises as energetic particles stream in one direction along magnetic field lines [136, 137, 14]. The effect is based on cascading of slab waves induced as a result of the instability development by the ambient turbulence. Thus the effect is not limited by suppressing of streaming instability. For instance, in [87] and [138] the suppression of gyroresonance instability by turbulence was considered.

The thorough numerical study of the suppression of the slab waves by Alfvenic turbulence was performed in [14], see Fig. 31] not only for the slab waves moving parallel to magnetic field, but also for waves moving at arbitrary angles to the mean magnetic field. The numerical simulations confirmed the theoretical expectations in the paper.

\subsection{Turbulent dynamo and high redshift physics}

Magnetic turbulent dynamo that we discussed in $\S 2$ is essential for understanding magnetic field in the early Universe. Indeed, as long as the Universe becomes ionized and highly conductive, the viscosity is also greatly reduced due to ions scattering in the magnetic fields [86, 88]. This creates high-Re environment which naturally produces turbulence. Despite a lot of discussion of early dynamo is concentrated on the initial field generation mechanisms, such as Biermann battery and its modifications [139], we now understand that in the limit of very high Re the level of the initial field is not very important. Instead, around 5\% of the energy of the turbulent cascade is deposited into magnetic energy due to high-Re small-scale dy- 

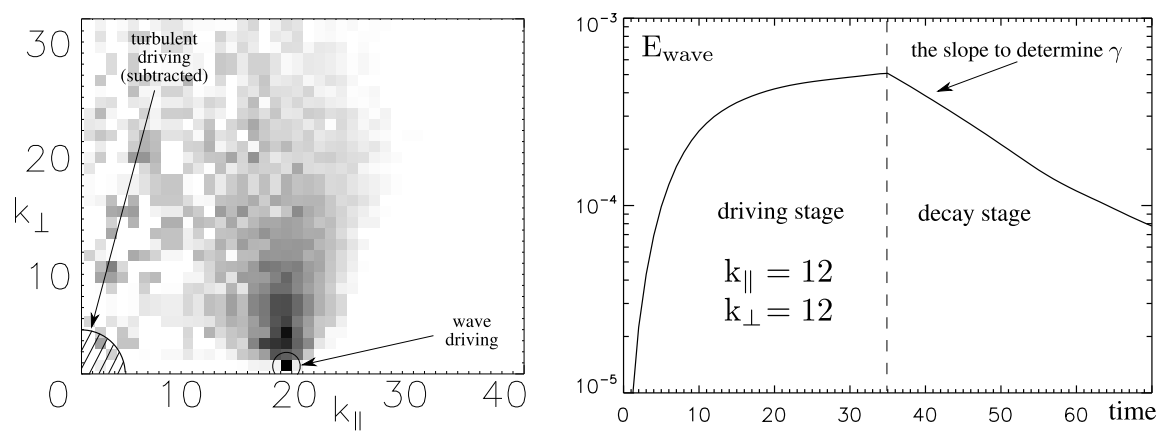

Fig. 31 The wave perturbation excited on top of existing turbulence exhibit exponential decay, as one expects a linear decay for a test perturbation in such perturbation simulation, despite the turbulence itself is strongly nonlinear. This linear decay rate is independent of amplitude, unlike nonlinear damping, so turbulence does not result in saturation of an instability but rather damps it completely or not. From [14].

namo [29]. The magnetic field, therefore, could get dynamically important at high redshifts. This will change the nature of many processes, for example we expect that magnetic field should be important in the process of formation of the first stars. These possibility is discussed in [140, 141], but we believe that the relative role of non-linear dynamo is even more important than it was presented in the latter study [142].

Acknowledgements AB was supported by Humboldt Fellowship. AL acknowledges the support of the NSF grant AST-1212096, the Vilas Associate Award as well as the support of the NSF Center for Magnetic Self- Organization. In addition, AL thanks the International Institute of Physics (Natal, Brazil) and the Observatoire de Nice for its hospitality during writing this review. Discussions with Ethan Vishniac and Greg Eyink are acknowledged. We thank Siyao Xu for reading the manuscript.

\section{References}

1. B.E. Goldstein, E.J. Smith, A. Balogh, T.S. Horbury, M.L. Goldstein, D.A. Roberts, GRL 22, 3393 (1995). DOI 10.1029/95GL03183

2. J.W. Armstrong, B.J. Rickett, S.R. Spangler, Astrophys. J.443, 209 (1995). DOI 10.1086/ 175515

3. A. Chepurnov, A. Lazarian, Astrophys. J.710, 853 (2010). DOI 10.1088/0004-637X/710/1/ 853

4. J. Cho, A. Lazarian, E.T. Vishniac, in Turbulence and Magnetic Fields in Astrophysics, Lecture Notes in Physics, Berlin Springer Verlag, vol. 614, ed. by E. Falgarone \& T. Passot (2003), Lecture Notes in Physics, Berlin Springer Verlag, vol. 614, pp. 56-98

5. B.G. Elmegreen, J. Scalo, Ann. Rep. A\&A 42, 211 (2004). DOI 10.1146/annurev.astro.41. 011802.094859

6. J.M. Stone, E.C. Ostriker, C.F. Gammie, ApJ 508, L99 (1998). DOI 10.1086/311718

7. E.C. Ostriker, J.M. Stone, C.F. Gammie, Astrophys. J.546, 980 (2001). DOI 10.1086/318290 
8. E. Vázquez-Semadeni, G.C. Gómez, A.K. Jappsen, J. Ballesteros-Paredes, R.F. González, R.S. Klessen, Astrophys. J.657, 870 (2007). DOI 10.1086/510771

9. C.F. McKee, E.C. Ostriker, Ann. Rep. A\&A 45, 565 (2007). DOI 10.1146/annurev.astro.45. 051806.110602

10. G.G. Howes, S.C. Cowley, W. Dorland, G.W. Hammett, E. Quataert, A.A. Schekochihin, Astrophys. J.651, 590 (2006). DOI 10.1086/506172

11. A.A. Schekochihin, S.C. Cowley, Turbulence and Magnetic Fields in Astrophysical Plasmas (Springer, 2007), pp. 85-+

12. J. Cho, A. Lazarian, ApJ 615, L41 (2004). DOI 10.1086/425215

13. A. Beresnyak, H. Yan, A. Lazarian, Astrophys. J.728, 60 (2011). DOI 10.1088/0004-637X/ $728 / 1 / 60$

14. A. Beresnyak, A. Lazarian, Astrophys. J.678, 961 (2008). DOI 10.1086/587052

15. S. Vainshtein, Y. Zeldovich, Physics-Uspekhi 15(2), 159 (1972)

16. E.T. Vishniac, J. Cho, Astrophys. J.550, 752 (2001). DOI 10.1086/319817

17. P.J. Käpylä, M.J. Korpi, A. Brandenburg, A\&A 500, 633 (2009). DOI 10.1051/0004-6361/ 200811498

18. F. Krause, K.H. Raedler, Mean-field magnetohydrodynamics and dynamo theory (Oxford: Pergamon Press, 1980)

19. A. Brandenburg, K. Subramanian, Phys. Rep. 417, 1 (2005). DOI 10.1016/j.physrep.2005. 06.005

20. A.P. Kazantsev, Soviet Journal of Experimental and Theoretical Physics 26, 1031 (1968)

21. R.H. Kraichnan, S. Nagarajan, Physics of Fluids 10, 859 (1967). DOI 10.1063/1.1762201

22. R.M. Kulsrud, S.W. Anderson, Astrophys. J.396, 606 (1992). DOI 10.1086/171743

23. R.A. Laing, A.H. Bridle, P. Parma, M. Murgia, MNRAS 391, 521 (2008). DOI 10.1111/j. 1365-2966.2008.13895.x

24. A. Schlüter, I. Biermann, Zeitschrift Naturforschung Teil A 5, 237 (1950)

25. N.E. Haugen, A. Brandenburg, W. Dobler, Phys. Rev. E70(1), 016308 (2004). DOI 10.1103/ PhysRevE.70.016308

26. J. Cho, E.T. Vishniac, A. Beresnyak, A. Lazarian, D. Ryu, Astrophys. J.693, 1449 (2009). DOI 10.1088/0004-637X/693/2/1449

27. D. Ryu, H. Kang, J. Cho, S. Das, Science 320, 909 (2008). DOI 10.1126/science.1154923

28. A. Beresnyak, T.W. Jones, A. Lazarian, Astrophys. J.707, 1541 (2009). DOI 10.1088/ 0004-637X/707/2/1541

29. A. Beresnyak, Phys. Rev. Lett.108(3), 035002 (2012). DOI 10.1103/PhysRevLett.108. 035002

30. P. Goldreich, S. Sridhar, Astrophys. J.438, 763 (1995). DOI 10.1086/175121

31. A. Beresnyak, Phys. Rev. Lett.106(7), 075001 (2011). DOI 10.1103/PhysRevLett.106. 075001

32. A. Beresnyak, A. Lazarian, Astrophys. J.702, 1190 (2009). DOI 10.1088/0004-637X/702/2/ 1190

33. H. Aluie, G.L. Eyink, Phys. Rev. Lett.104(8), 081101 (2010). DOI 10.1103/PhysRevLett. 104.081101

34. A. Alexakis, P.D. Mininni, A. Pouquet, Phys. Rev. E72(4), 046301 (2005). DOI 10.1103/ PhysRevE.72.046301

35. G.L. Eyink, Physica D Nonlinear Phenomena 207, 91 (2005). DOI 10.1016/j.physd.2005. 05.018

36. A. Beresnyak, A. Lazarian, Astrophys. J.702, 460 (2009). DOI 10.1088/0004-637X/702/1/ 460

37. A. Kolmogorov, Akademiia Nauk SSSR Doklady 30, 301 (1941)

38. P. Iroshnikov, Soviet Astronomy 7, 566 (1964)

39. R. Kraichnan, Physics of Fluids 8, 1385 (1965)

40. J. Cho, A. Lazarian, MNRAS 345, 325 (2003). DOI 10.1046/j.1365-8711.2003.06941.x

41. A. Beresnyak, A. Lazarian, J. Cho, ApJ 624, L93 (2005). DOI 10.1086/430702

42. B.B. Kadomtsev, O.P. Pogutse, Soviet Journal of Experimental and Theoretical Physics 38, 283 (1974) 
43. H.R. Strauss, Physics of Fluids 19(1), 134 (1976). DOI 10.1063/1.861310. URL http://link.aip.org/link/?PFL/19/134/1

44. A. Beresnyak, A. Lazarian, Astrophys. J.682, 1070 (2008). DOI 10.1086/589428

45. A. Lazarian, E.T. Vishniac, Astrophys. J.517, 700 (1999). DOI 10.1086/307233

46. J. Cho, E.T. Vishniac, Astrophys. J.539, 273 (2000). DOI 10.1086/309213

47. J. Maron, P. Goldreich, Astrophys. J.554, 1175 (2001). DOI 10.1086/321413

48. J. Cho, A. Lazarian, E.T. Vishniac, Astrophys. J.564, 291 (2002). DOI 10.1086/324186

49. W.C. Müller, R. Grappin, Phys. Rev. Lett.95(11), 114502 (2005). DOI 10.1103/PhysRevLett. 95.114502

50. S. Galtier, A. Pouquet, A. Mangeney, Physics of Plasmas 12(9), 092310 (2005). DOI 10. $1063 / 1.2052507$

51. S. Boldyrev, ApJ 626, L37 (2005). DOI 10.1086/431649

52. G. Gogoberidze, Physics of Plasmas 14(2), 022304 (2007). DOI 10.1063/1.2437753

53. S. Boldyrev, Phys. Rev. Lett.96(11), 115002 (2006). DOI 10.1103/PhysRevLett.96.115002

54. A. Beresnyak, A. Lazarian, ApJ 640, L175 (2006). DOI 10.1086/503708

55. A.A. Schekochihin, S.C. Cowley, W. Dorland, G.W. Hammett, G.G. Howes, E. Quataert, T. Tatsuno, ApJ 182, 310 (2009). DOI 10.1088/0067-0049/182/1/310

56. S. Galtier, S.V. Nazarenko, A.C. Newell, A. Pouquet, Journal of Plasma Physics 63, 447 (2000). DOI 10.1017/S0022377899008284

57. U. Frisch, Turbulence. The legacy of A. N. Kolmogorov. (Cambridge Univ Pr, 1995)

58. A.S. Monin, A.M. Iaglom, Statistical fluid mechanics: Mechanics of turbulence. Volume 2 /revised and enlarged edition/ (Cambridge, Mass., MIT Press, 1975)

59. D. Biskamp, Magnetohydrodynamic Turbulence (Cambridge University Press, 2003)

60. H. Politano, A. Pouquet, Phys. Rev. E 57, R21 (1998). DOI 10.1103/PhysRevE.57.R21. URL http://link.aps.org/doi/10.1103/PhysRevE.57.R21

61. P.K. Yeung, Y. Zhou, Phys. Rev. E56, 1746 (1997). DOI 10.1103/PhysRevE.56.1746

62. T. Gotoh, D. Fukayama, T. Nakano, Physics of Fluids 14, 1065 (2002)

63. Y. Kaneda, T. Ishihara, M. Yokokawa, K. Itakura, A. Uno, Physics of Fluids 15, L21 (2003). DOI 10.1063/1.1539855

64. A. Beresnyak, A. Lazarian, ApJ 722, L110 (2010). DOI 10.1088/2041-8205/722/1/L110

65. A. Beresnyak, MNRAS 422, 3495 (2012). DOI 10.1111/j.1365-2966.2012.20859.x

66. A. Beresnyak, ArXiv e-prints (2014)

67. J. Mason, F. Cattaneo, S. Boldyrev, Phys. Rev. Lett.97(25), 255002 (2006). DOI 10.1103/ PhysRevLett.97.255002

68. H. Yan, A. Lazarian, Phys. Rev. Lett.89, 281102 (2002). DOI 10.1103/PhysRevLett.89. 281102

69. M. Dobrowolny, A. Mangeney, P. Veltri, Physical Review Letters 45, 144 (1980). DOI 10.1103/PhysRevLett.45.144

70. W.H. Matthaeus, D. Montgomery, Annals of the New York Academy of Sciences 357, 203 (1980). DOI 10.1111/j.1749-6632.1980.tb29687.x

71. R. Grappin, J. Leorat, A. Pouquet, A\&A 126, 51 (1983)

72. A. Pouquet, P.L. Sulem, M. Meneguzzi, Physics of Fluids 31, 2635 (1988). DOI 10.1063/1. 866541

73. A. Pouquet, U. Frisch, J. Léorat, Journal of Fluid Mechanics 77(02), 321 (1976)

74. Y. Lithwick, P. Goldreich, S. Sridhar, Astrophys. J.655, 269 (2007). DOI 10.1086/509884

75. B.D.G. Chandran, Astrophys. J.685, 646 (2008). DOI 10.1086/589432

76. J.C. Perez, S. Boldyrev, Phys. Rev. Lett.102(2), 025003 (2009). DOI 10.1103/PhysRevLett. 102.025003

77. J. Cho, A. Lazarian, Phys. Rev. Lett.88(24), 245001 (2002). DOI 10.1103/PhysRevLett.88. 245001

78. G.P. Zank, W.H. Matthaeus, Physics of Fluids 5, 257 (1993). DOI 10.1063/1.858780

79. G. Kowal, A. Lazarian, Astrophys. J.720, 742 (2010). DOI 10.1088/0004-637X/720/1/742

80. A. Beresnyak, H. Xu, H. Li, R. Schlickeiser, Astrophys. J.771, 131 (2013). DOI 10.1088/ 0004-637X/771/2/131 
81. B.T. Draine, A. Lazarian, Astrophys. J.512, 740 (1999). DOI 10.1086/306809

82. J. Cho, A. Lazarian, E.T. Vishniac, ApJ 566, L49 (2002). DOI 10.1086/339453

83. J. Cho, A. Lazarian, E.T. Vishniac, Astrophys. J.595, 812 (2003). DOI 10.1086/377515

84. A. Lazarian, E.T. Vishniac, J. Cho, Astrophys. J.603, 180 (2004). DOI 10.1086/381383

85. J. Cho, E.T. Vishniac, Astrophys. J.538, 217 (2000). DOI 10.1086/309127

86. A.A. Schekochihin, S.C. Cowley, Physics of Plasmas 13(5), 056501 (2006). DOI 10.1063/ 1.2179053

87. A. Lazarian, A. Beresnyak, MNRAS 373, 1195 (2006). DOI 10.1111/j.1365-2966.2006. 11093.x

88. A.A. Schekochihin, S.C. Cowley, R.M. Kulsrud, M.S. Rosin, T. Heinemann, Physical Review Letters 100(8), 081301 (2008). DOI 10.1103/PhysRevLett.100.081301

89. R. Santos-Lima, E.M. de Gouveia Dal Pino, G. Kowal, D. Falceta-Gonçalves, A. Lazarian, M.S. Nakwacki, Astrophys. J.781, 84 (2014). DOI 10.1088/0004-637X/781/2/84

90. J. Cho, Astrophys. J.621, 324 (2005). DOI 10.1086/427493

91. C. Thompson, O. Blaes, Phys. Rev. D57, 3219 (1998). DOI 10.1103/PhysRevD.57.3219

92. J. Cho, A. Lazarian, Astrophys. J.780, 30 (2014). DOI 10.1088/0004-637X/780/1/30

93. M. Lyutikov, A. Lazarian, SSR178, 459 (2013). DOI 10.1007/s11214-013-9989-2

94. R. Benzi, M.V. Struglia, R. Tripiccione, in eprint arXiv:chao-dyn/9509018 (1995), p. 9018

95. Z.S. She, E. Leveque, Physical Review Letters 72, 336 (1994). DOI 10.1103/PhysRevLett. 72.336

96. B. Dubrulle, Physical Review Letters 73, 959 (1994). DOI 10.1103/PhysRevLett.73.959

97. W.C. Müller, D. Biskamp, Physical Review Letters 84, 475 (2000). DOI 10.1103/ PhysRevLett.84.475

98. S. Boldyrev, Astrophys. J.569, 841 (2002). DOI 10.1086/339403

99. A.G. Kritsuk, M.L. Norman, P. Padoan, R. Wagner, Astrophys. J.665, 416 (2007). DOI $10.1086 / 519443$

100. P. Padoan, R. Jimenez, M. Juvela, Å. Nordlund, ApJ 604, L49 (2004). DOI 10.1086/383308

101. A. Lazarian, L. Vlahos, G. Kowal, H. Yan, A. Beresnyak, E.M. de Gouveia Dal Pino, SSRp. 86 (2012). DOI 10.1007/s11214-012-9936-7

102. D. Biskamp, Magnetic Reconnection in Plasmas (UK: Cambridge, 2000)

103. E. Priest, T. Forbes, UK: Cambridge (2000)

104. T.W. Speiser, Plan. Space Sciences18, 613 (1970). DOI 10.1016/0032-0633(70)90136-4

105. A.R. Jacobson, R.W. Moses, Phys. Rev. A29, 3335 (1984). DOI 10.1103/PhysRevA.29.3335

106. W.H. Matthaeus, S.L. Lamkin, Physics of Fluids 28, 303 (1985). DOI 10.1063/1.865147

107. W.H. Matthaeus, S.L. Lamkin, Physics of Fluids 29, 2513 (1986). DOI 10.1063/1.866004

108. G.L. Eyink, A. Lazarian, E.T. Vishniac, Astrophys. J.743, 51 (2011). DOI 10.1088/ 0004-637X/743/1/51

109. G. Kowal, A. Lazarian, E.T. Vishniac, K. Otmianowska-Mazur, Astrophys. J.700, 63 (2009). DOI 10.1088/0004-637X/700/1/63

110. G. Kowal, A. Lazarian, E.T. Vishniac, K. Otmianowska-Mazur, Nonlinear Processes in Geophysics 19, 297 (2012). DOI 10.5194/npg-19-297-2012

111. E.T. Vishniac, A. Lazarian, Astrophys. J.511, 193 (1999). DOI 10.1086/306643

112. A. Lazarian, in Magnetic Fields in the Universe: From Laboratory and Stars to Primordial Structures., American Institute of Physics Conference Series, vol. 784, ed. by E.M. de Gouveia dal Pino, G. Lugones, A. Lazarian (2005), American Institute of Physics Conference Series, vol. 784, pp. 42-53. DOI 10.1063/1.2077170

113. G.L. Eyink, Phys. Rev. E83(5), 056405 (2011). DOI 10.1103/PhysRevE.83.056405

114. A. Lazarian, A. Esquivel, R. Crutcher, Astrophys. J.757, 154 (2012). DOI 10.1088/ 0004-637X/757/2/154

115. A. Lazarian, SSR(2013). DOI 10.1007/s11214-013-0031-5

116. G. Eyink, E. Vishniac, C. Lalescu, H. Aluie, K. Kanov, K. Bürger, R. Burns, C. Meneveau, A. Szalay, Nature497, 466 (2013). DOI 10.1038/nature12128

117. D.B. Melrose, Plasma astrohysics. Nonthermal processes in diffuse magnetized plasmas Vol.1: The emission, absorption and transfer of waves in plasmas; Vol.2: Astrophysical applications (New York: Gordon and Breach, 1980) 
118. B.D.G. Chandran, Physical Review Letters 85, 4656 (2000). DOI 10.1103/PhysRevLett.85. 4656

119. H. Yan, A. Lazarian, B.T. Draine, Astrophys. J.616, 895 (2004). DOI 10.1086/425111

120. H. Yan, A. Lazarian, Astrophys. J.677, 1401 (2008). DOI 10.1086/533410

121. G. Brunetti, A. Lazarian, MNRAS 378, 245 (2007). DOI 10.1111/j.1365-2966.2007.11771. $\mathrm{X}$

122. R. Schlickeiser, Cosmic Ray Astrophysics (Springer, 2002)

123. E.M. de Gouveia Dal Pino, A. Lazarian, A\&A 441, 845 (2005). DOI 10.1051/0004-6361: 20042590

124. G. Kowal, E.M. de Gouveia Dal Pino, A. Lazarian, Physical Review Letters 108(24), 241102 (2012). DOI 10.1103/PhysRevLett.108.241102

125. A. Lazarian, in SINS - Small Ionized and Neutral Structures in the Diffuse Interstellar Medium, Astronomical Society of the Pacific Conference Series, vol. 365, ed. by M. Haverkorn, W.M. Goss (2007), Astronomical Society of the Pacific Conference Series, vol. 365 , p. 324

126. C. Heiles, Astrophys. J.481, 193 (1997). DOI 10.1086/304033

127. S. Stanimirović, J.M. Weisberg, A. Hedden, K. Devine, T. Green, S.B. Anderson, Astrophysics and Space Science292, 103 (2004). DOI 10.1023/B:ASTR.0000045005.36554.36

128. P. Goldreich, S. Sridhar, ApJ 640, L159 (2006). DOI 10.1086/503668

129. A.A. Schekochihin, S.C. Cowley, S.F. Taylor, J.L. Maron, J.C. McWilliams, Astrophys. J.612, 276 (2004). DOI 10.1086/422547

130. A. Lazarian, M. Opher, Astrophys. J.703, 8 (2009). DOI 10.1088/0004-637X/703/1/8

131. E. Falgarone, G. Pineau des Forets, E. Roueff, A\&A 300, 870 (1995)

132. K. Joulain, E. Falgarone, G. Pineau des Forets, D. Flower, A\&A 340, 241 (1998)

133. B. Godard, E. Falgarone, G.A. Pineau Des Forêts, A\&A 495, 847 (2009). DOI 10.1051/ 0004-6361:200810803

134. B. Godard, E. Falgarone, G. Pineau Des Forêts, A\&A in press (2014)

135. G. Kowal, A. Lazarian, A. Beresnyak, Astrophys. J.658, 423 (2007). DOI 10.1086/511515

136. H. Yan, A. Lazarian, Astrophys. J.614, 757 (2004). DOI 10.1086/423733

137. A.J. Farmer, P. Goldreich, Astrophys. J.604, 671 (2004). DOI 10.1086/382040

138. H. Yan, A. Lazarian, Astrophys. J.731, 35 (2011). DOI 10.1088/0004-637X/731/1/35

139. A. Lazarian, A\&A 264, 326 (1992)

140. J. Schober, D. Schleicher, C. Federrath, S. Glover, R.S. Klessen, R. Banerjee, Astrophys. J.754, 99 (2012). DOI 10.1088/0004-637X/754/2/99

141. J. Schober, D.R.G. Schleicher, R.S. Klessen, A\&A 560, A87 (2013). DOI 10.1051/ 0004-6361/201322185

142. S. Xu, A. Lazarian, in preparation (2014) 